\title{
An Investigation of the Usefulness of Extremely Low-Frequency Electromagnetic Measurements for Treaty Verification
}

\author{
Jerry J. Sweeney
}

Manuscript date: January 1989 


\section{DISCLAIMER}

This report was prepared as an account of work sponsored by an agency of the United States Government. Neither the United States Government nor any agency Thereof, nor any of their employees, makes any warranty, express or implied, or assumes any legal liability or responsibility for the accuracy, completeness, or usefulness of any information, apparatus, product, or process disclosed, or represents that its use would not infringe privately owned rights. Reference herein to any specific commercial product, process, or service by trade name, trademark, manufacturer, or otherwise does not necessarily constitute or imply its endorsement, recommendation, or favoring by the United States Government or any agency thereof. The views and opinions of authors expressed herein do not necessarily state or reflect those of the United States Government or any agency thereof. 


\section{DISCLAIMER}

Portions of this document may be illegible in electronic image products. Images are produced from the best available original document. 



\section{Contents}

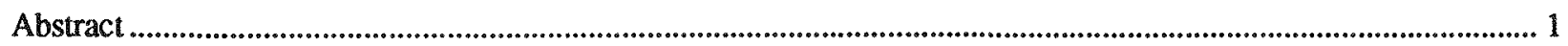

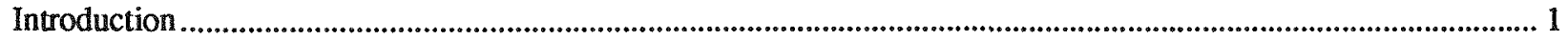

Natural and Man-Made Sources of ELF Signals .................................................................................... 2

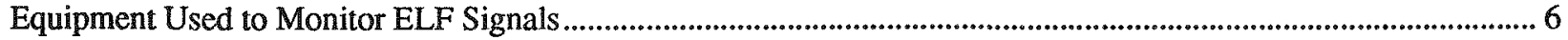

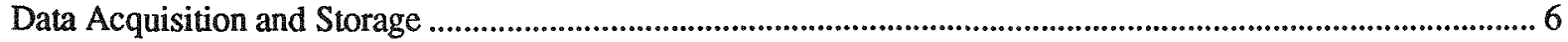

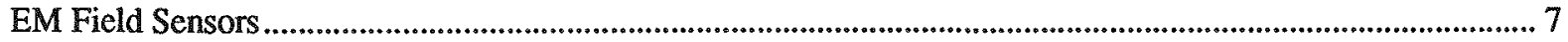

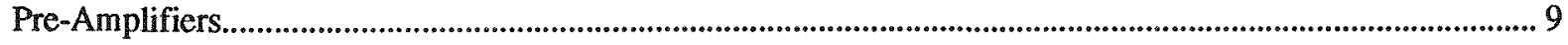

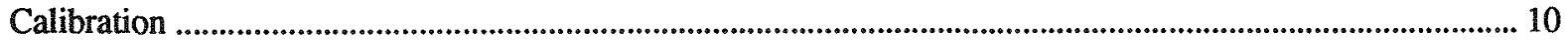

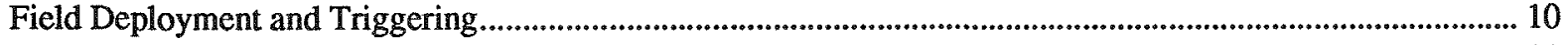

ELF Monitoring Results from Underground Nuclear Tests ......................................................................... 11

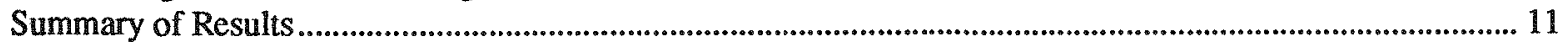

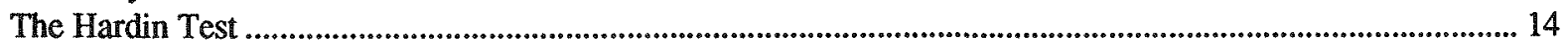

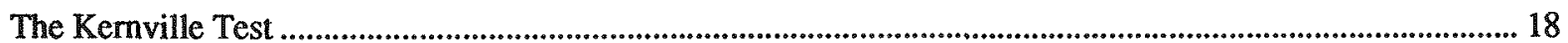

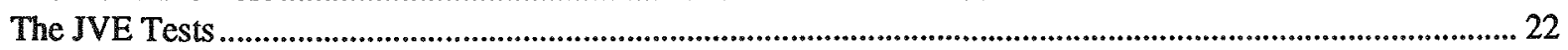

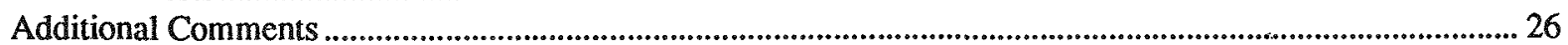

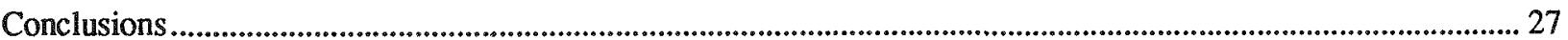

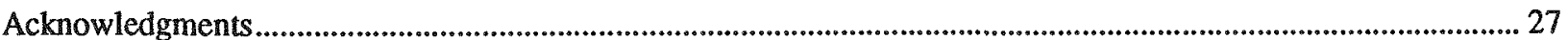

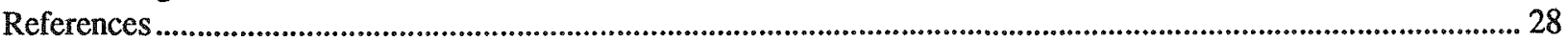

Appendix A: Source Modeling of Event Induced ELF Radiation ....................................................................... 29.

Appendix B: Excitation of the Earth-Ionosphere Cavity by an Underground Nuclear Test .................................... 49 


\title{
An Investigation of the Usefulness of Extremely Low-Frequency Electromagnetic Measurements for Treaty Verification
}

\begin{abstract}
From FY 1986 through FY 1988, we monitored extremely low-frequency (ELF) electromagnetic (EM) fields to determine whether these signals could be detected from underground nuclear explosions. Signals clearly related to underground tests were detected only when the ELF field sensors were located within $10 \mathrm{~km}$ of surface ground zero. Theoretical analysis, based on the results of these measurements, shows that the ELF impulse signals from underground nuclear tests are of longer duration than those from lightning sources and are, therefore, less efficient in exciting resonances in the earth-ionosphere cavity, even though the source strength for each may be the same. Thus, ELF signals from underground nuclear tests with yields of $<150 \mathrm{kT}$ are generally lower than the background signals caused by worldwide lightning activity. Our conclusion is that ELF monitoring probably will not be useful for detecting underground nuclear tests at distances $>10 \mathrm{~km}$ from the tests.
\end{abstract}

\section{Introduction}

The purpose of this report is to document approximately three ycars of research aimed at determining whether EM measurements in the ELF range can be applied to regional or global monitoring of underground nuclear tests and, therefore, can be of use in treaty verification efforts. The initial research, including measurements made during three underground nuclear tests at the Nevada Test Site (NTS), was carried out by H. M. Buettner of the Engineering Research Division of the Electrical Engineering Department at Lawrence Livermore National Laboratory (LLNL) during the last quarter of FY 1985. In FY 1986, the Verification Research Program in the LLNL Earth Sciences Department received funding for this project; P. W. Rodgers of the Seismology and Applied Geophysics Group was placed in charge of the project. Rodgers retired during the early part of FY 1987; the author, also a member of the Seismology and Applied Geophysics Group, took over the project and directed it until its completion at the end of FY 1988. During the last two years of the project, D. D. Sentman of the Institute of Geophysics and Planetary Physics (IGPP) at the University of California, Los Angeles (UCLA) was subcontracted to assist in ELF equipment design and to field an ELF measurement station at NTS. Sentman has been carrying out research on the characteristics of ELF signals for several years and has made several contributions to the theoretical knowledge on the subject. ${ }^{1-4}$ In the later part of FY 1987 , Sentman received additional funding from Los Alamos National Laboratory through the IGPP that allowed him to field a second ELF station at NTS. The data from these UCLA stations are an essential part of this study.

The treaty verification community has always considered ELF EM measurements to have a relatively low probability for success, but they funded the work because the potential payoff for such measurements would be very high. By their nature, EM 
measurements complement seismic measurements because they detect a different type of energy that travels (in vacuo) at the speed of light.

The EM field of a nuclear explosion is caused by the motion of high-energy electrons (Compton electrons) generated in the fireball and by magnetohydrodynamic effects. The sources of EM emissions from earthquakes and, possibly, from chemical explosions are different than those from nuclear explosions. Though as yet unproven, a high probability exists that EM signals generated by earthquakes, chemical explosions, and underground nuclear explosions are fundamentally different; therefore, the ability to detect and measure these differences might allow us to discriminate between these three phenomena. For instance, if a nuclear explosion is detonated in an open underground cavity (an uncoupled explosion), there is a chance that the EM signals emanating from the event are enhanced because the fields are created in a low-conductivity cavity rather than in relatively higher-conductivity earth materials. In addition, the seismic signal from an uncoupled explosion is degraded because mechanical coupling of the seismic energy is less effective than in an ordinary borehole test. This diffe:ence in energy response (enhanced vs degraded) for uncoupled explosions could thus be exploited for use in detection and discrimination.

Because there is no delay in the EM signal, it could be used to determine the precise timing of a nuclear explosion anywhere in the world. In other words, EM measurements could be used to detect underground nuclear tests at teleseismic distances anywhere almost instantaneously. With an accurate source time, the seismologist can be much more exact in determining the location of the event.

In this study, we chose to investigate the ELF band ( 0 to $3000 \mathrm{~Hz}$ ) of EM energy for the following reasons. First, at ELF frequencies, the wavelength in free space is $10 \mathrm{~km}$ or greater. Second, the penetration depth of the EM signals into the conductive earth is maximum at these frequencies. Therefore, because these frequencies are the least attenuated by passage through the ground, this energy is more efficiently coupled from an underground source into the atmosphere than are higher frequencies. Third, Schumann and others ${ }^{5,6}$ demonstrated that the earth and ionosphere act as a concenuric spherical waveguide at ELF frequencies and trap the EM energy generated by lightning discharges; this energy can be detected worldwide. Thus, ELF energy generated by an underground nuclear test, if it were large enough, could couple into the earthionosphere waveguide and could also be detected worldwide. Last, the lower part of the ELF band $(0$ to $30 \mathrm{~Hz})$ is in the frequency range commonly monitored by seismic equipment; therefore we can employ digital sampling techniques and equipment that are already in use by seismologists and readily available at LLNL to record and store ELF EM data.

In this report, I discuss the nature of the ELF background signals (or "noise" to those interested in signals related to discrete events) and summarize results from unclassified ELF investigations that are pertinent to this research. I also describe our ELF measurement equipment, summarize the results of the previous three and a half years work at LLNL, and draw conclusions from these results. Note that the purpose of this research was to investigate the potential of using ELF EM monitoring to detect underground nuclear explosions. The purpose was not to use ELF measurements to determine the yield of the device or to understand the fundamental nature of the source of the EM energy, although I do make some comments about these matters in my analysis of the data.

\section{Natural and Man-Made Sources of ELF Signals}

W. O. Schumann's original theory states that the surface of the earth and the ionosphere can trap EM energy in discrete frequency bands called Schumann resonances.5 In 1960, Balser and Wagner confirmed this theory with their measurements. They found the fundamental Schumann resonant frequencies to be $8,14.1,20.3,26.4$, and $32.5 \mathrm{~Hz}$ with the resonant cavity $Q$ at $8 \mathrm{~Hz}$ being -4 (i.e., the half-power bandwidth is $\sim 2 \mathrm{~Hz}$ ). At $10 \mathrm{~Hz}$, the free-space wavelength is $30,000 \mathrm{~km}$. The height of the ionosphere and, hence, the distance between the cavity boundaries is $\sim 100 \mathrm{~km}$. Therefore, the freespace wavelength for ELF resonances is much greater than the space between the conductors; in this case, only the lowest order transverse magnetic mode can propagate. ${ }^{3}$ The transverse electric modes have -400 to 600 times more attenuation than the transverse magnetic modes at $10 \mathrm{~Hz}$ and, thus, are not important. $^{7}$ Therefore, in the first approximation, the earth-ionosphere cavity with dipole excitation has 
azimuthal symmetry, and the only field components are radial and tangential electric, and tangential magnetic. At the earth's surface, the most important field components of the lowest order modes are the vertical electric and the two orthogonal magnetic fields.

Examples of the Schumann resonances in the frequency domain are shown in Fig. 1. Here, the power spectral densily is calculated from $180 \mathrm{~s}$ of data; the peaks of the Schumann modes (vertical electric $E_{\mathrm{z}}$, vertical magnetic $B_{\mathrm{n}}$, and east magnetic $B_{\mathrm{e}}$ ) can be readily seen in this figure. The center frequency and shape of the Schumann resonances depend on the following:

- The electron density (and thus the conductivity) of the ionosphere.

- The shape of the ionosphere, which has large changes on the diumal scale and smaller changes on smaller time scales.

- The location and character (orientation and strength) of the energy sources.

Over periods of tens of seconds or less, the nature of Schumann resonances can change considerably. Figure 2 shows the power-density spectra for the north magnetic ELF component taken over times ranging from 10 to $180 \mathrm{~s}$. Notice how the Schumann components of the energy become better defined when the data is averaged over longer times.

Peaks in the Schumann resonance energy can be correlated in time over daily cycles with peaks in worldwide thunderstorm activity, especially in Africa, Asia, and the Americas. Peaks in ELF background signal levels have also been correlated with regional thunderstorm activity monitored by low-frequency EM measurements. ${ }^{7}$

A current of electricity discharging from the ground into the underside of a cloud acts as vertical electric-dipole source. The cavity is generally excited simultaneously by many thunderstorms, but occasionally a strong impulse produced by a single very high discharge can be detected (see the right side of Fig. 3). The "ringing" seen in the $E_{\mathrm{Z}}$ component is close to the first Schumann frequency, $\sim 8 \mathrm{~Hz}$. The double impulses seen in the magnetic components $\left(B_{\mathrm{n}}\right.$ and $B_{\mathrm{e}}$ ) are probably due to a double source; most lightning bolts have multiple discharges with return strokes occurring from 50 to $150 \mathrm{~ms}$ after the initial discharge. These strong individually identified bolis are usually referred to as $Q$-bursts ${ }^{8}$ because the tapering off of the impulse signal can be used to measure the $Q$ of the earth-ionosphere cavity at the time the bolt occurred. The weaker $Q$-burst at $\sim 66.25 \mathrm{~s}$ in Fig. 3 is more typical and is due to a much smaller bolt of lightning. Typical lightning strokes are several kilometers in length with peak currents of $20 \mathrm{kA}$ for a few microseconds 9 ; thus, an equivalent vertical-dipole source has a strength of $\sim 2 \times 10^{7} \mathrm{~A} \cdot \mathrm{m}$.

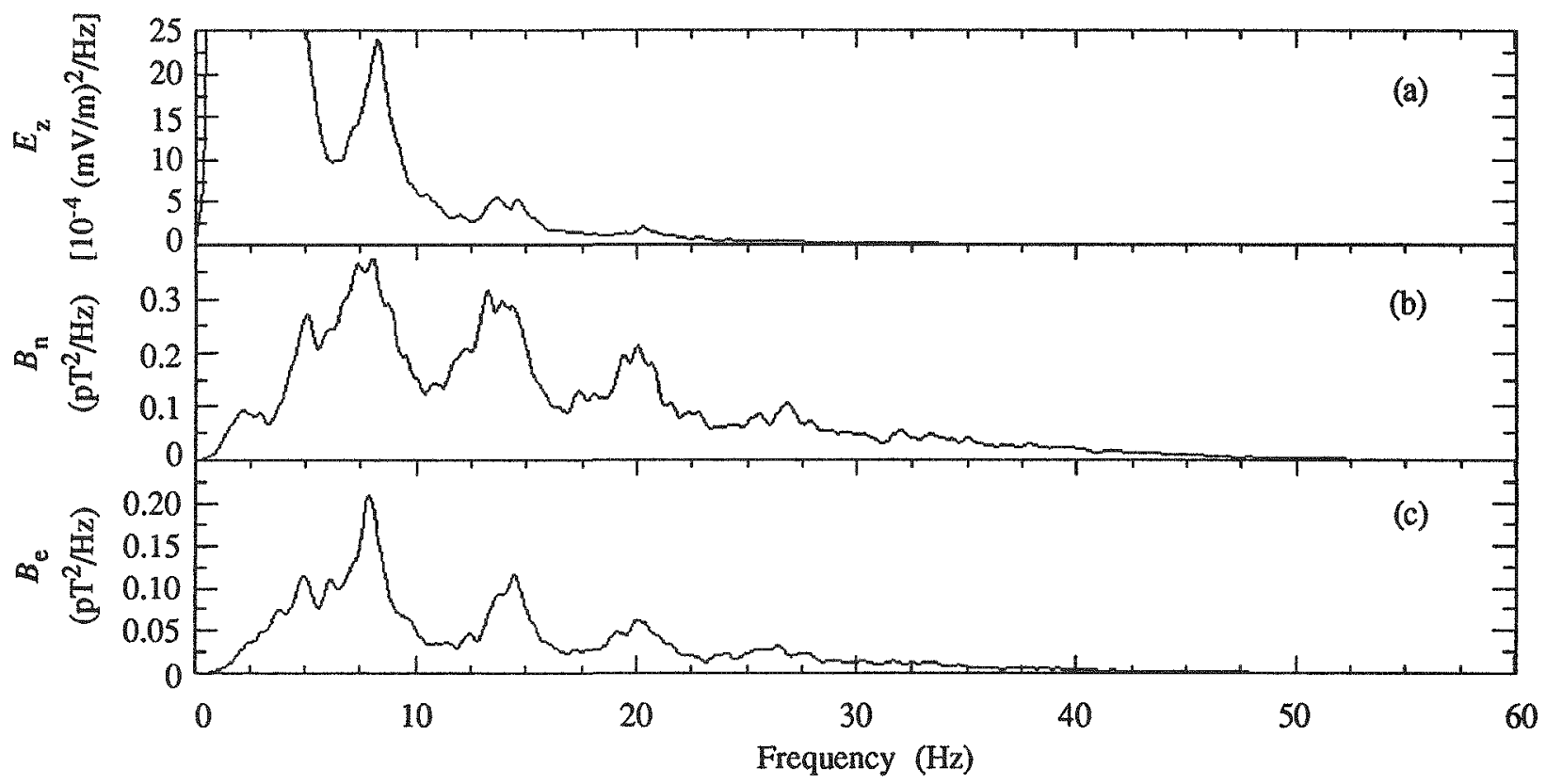

Figure 1. Comparison of power-density spectra of the three fundamental Schumann components: (a) $E_{\mathrm{Z}}$, (b) $B_{\mathrm{n}}$, and (c) $B_{\mathrm{e}^{\circ}}$ The spectra were computed from $180 \mathrm{~s}$ of typical background signal data. 


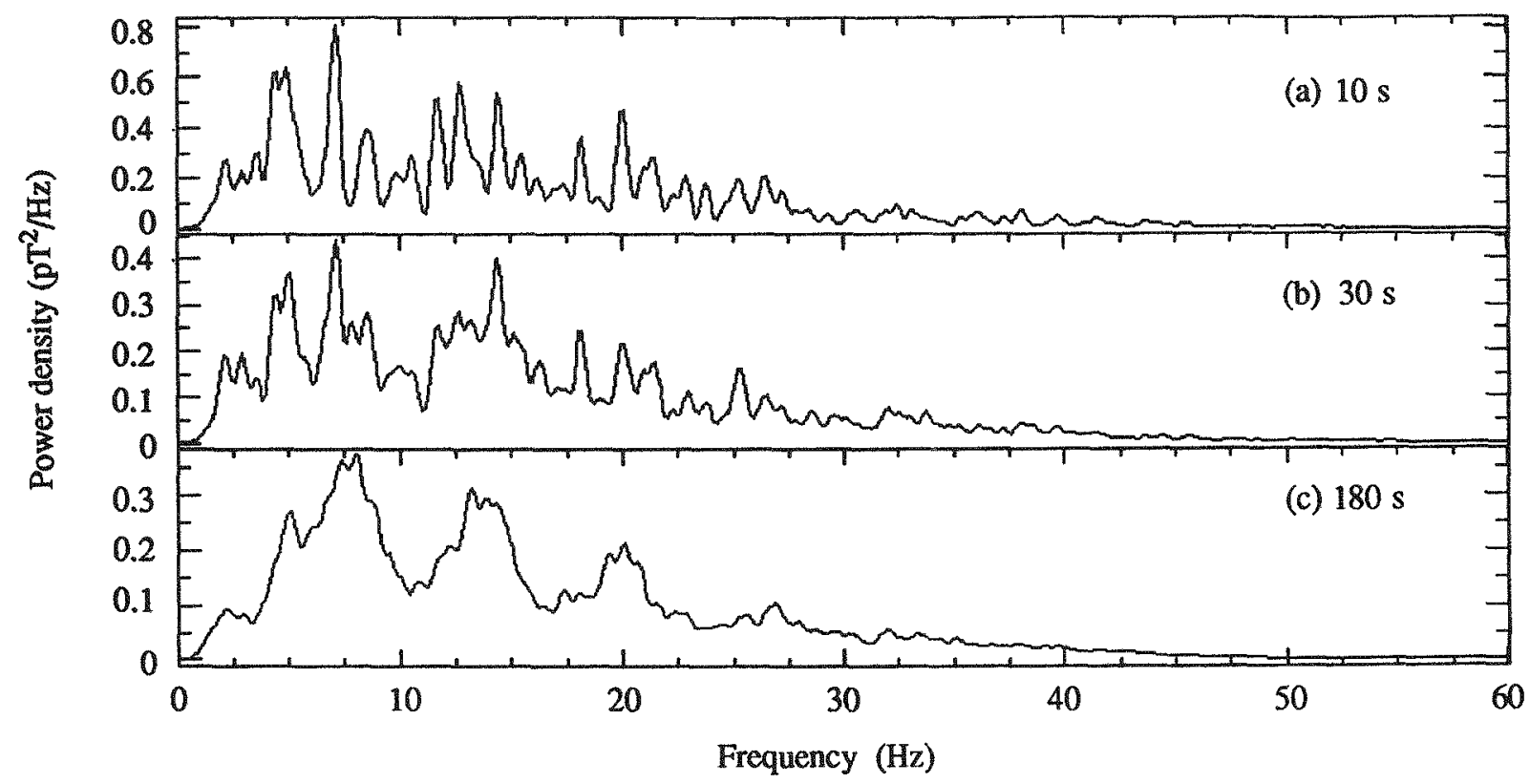

Figure 2. Power-density spectra of north-magnetic-component ELF data, showing the effect of averaging time on the nature of the spectra. (a) $10 \mathrm{~s}$ of background data were used to compute the spectra, (b) $30 \mathrm{~s}$, and (c) $180 \mathrm{~s}$.

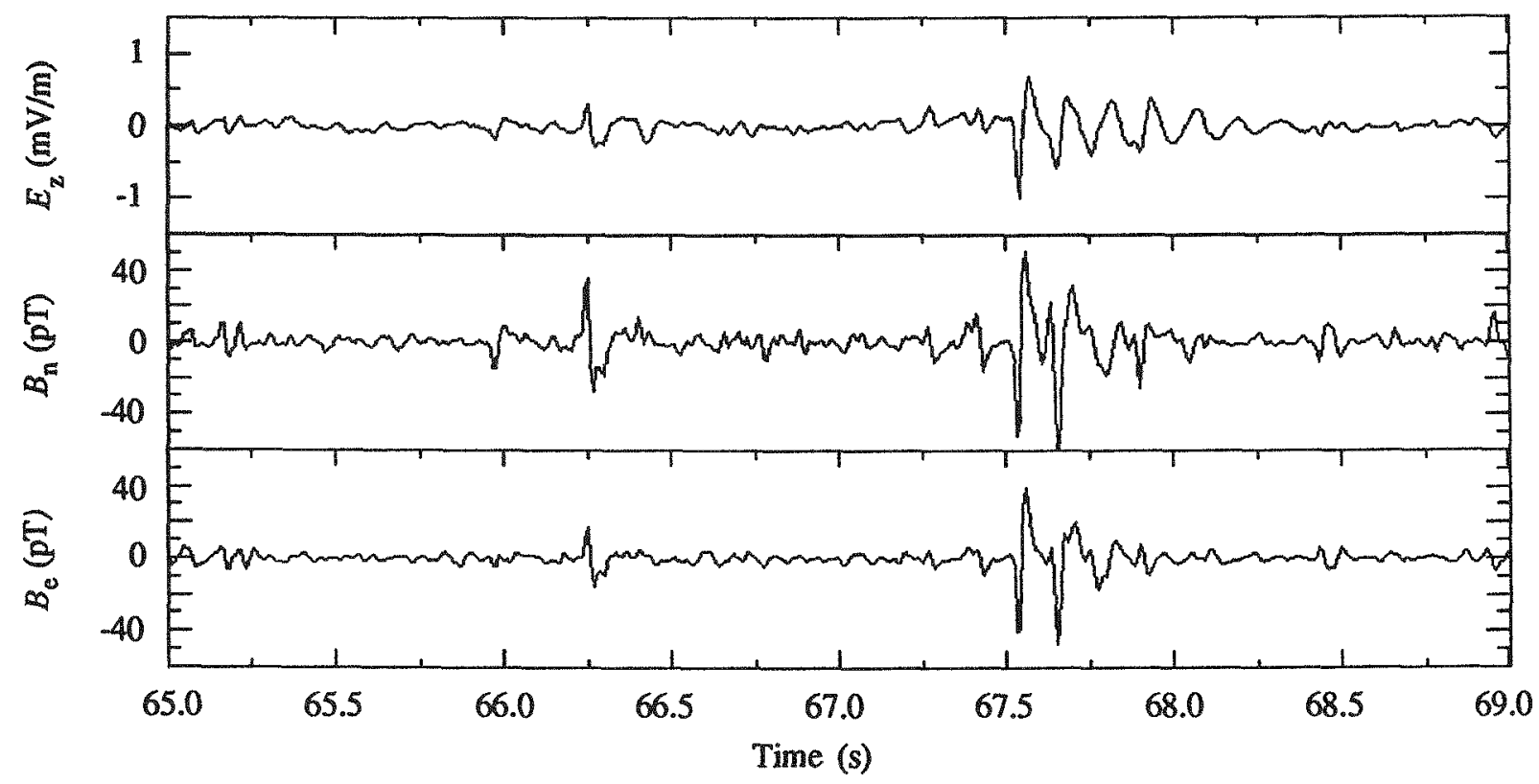

Figure 3. ELF Schumanm component data from the UCLA FF station at NTS. A typical $Q$-burst, emanating from distant lightning, is seen $66.25 \mathrm{~s}$ after the start of the file. A very strong $Q$-burst, showing an often seen double pulse with ringing at 7 to $8 \mathrm{~Hz}$, occurs $67.5 \mathrm{~s}$ into the file. 
The strong $Q$-bursts like those shown in Fig. 3 and even some of the weaker bursts are important because they essentially occur simuliancously at distant (thousands of kilometers from the source) measurement stations. Thus, $Q$-bursts can be used to check the accuracy of the respective time references, when ELF measurements are taken at different stations. Examples of this will be discussed in more detail below.

Perturbations in the character of the Schumann resonances caused by an atmospheric nuclear explosion were observed by Balser and Wagner ${ }^{10}$ in 1963. They observed, using equipment located in Massachusetts, an immediate decrease of $\sim 10 \%$ in the frequency of the $8-\mathrm{Hz}$ Schumann mode at the detonation time of a megaton-range device hundreds of kilometers above Johnston Island. This perturbation of the resonant frequency from the normal diurnal trend lasted for several hours. They also measured a very strong prompt signal, with a frequency of $4 \mathrm{~Hz}$ or less, that saturated their equipment. This strong prompt signal was expected because Compton electrons moving away from the detonation, with the earth's surface acting as a conducting half plane, form a very strong vertical electric dipole that creates this prompt signal. The pressure wave in the atmosphere causes a large perturbation in the shape of the ionosphere, thus causing a shift in the Schumann resonances.

Other observations of ELF signals from both atmospheric and underground nuclear tests were reported in the unclassified literature by Zablocki11 and by Malik et al.12 Zablocki measured low-frequency transients using large horizontal magnetic loops (that were radial and tangential to a reference direction from the measurement site to ground zero) and using a vertical wire in a borehole during five underground and two low-level atmospheric tests. $\mathrm{He}$ investigated two theories to explain the generation of the EM signal:

- The distortion of Earth's magnetic field by the conductive fireball; this is generally known as the magnetic bubble theory.

- The development of a nonsymmetrical electron flux surrounding the explosion; this is generally known as the electric dipole theory.

These two mechanisms of field generation have very different EM behavior that would be manifest in the types of near-field signals recorded. Zablocki worked out approximate solutions for the near-field equations for each of these and compared the characteristics of the theoretical fields with those measured. For this project, Jerry Burke of the LLNL Electrical Engineering Department checked Zablocki's calculations and found some minor errors; however, Zablocki's basic conclusions remain valid. (Burke's calculations of these fields for various geometries are given in Appendix A).

Table 1 lists some general conclusions from Zablocki's analysis of the near-field equations; it shows which field components are expected from various source types. The directions refer to a vector pointing from the measurement point to surface ground zero. In general, all of the fields are very strong functions of the inverse distance from the source (see Appendix A) and most of them rapidly die. These field equations also assume that the source is located at the earth's surface; for buried sources (e.g., underground nuclear tests), the fields due to electric dipole sources are further attenuated by a factor of $10^{6}$ or more because of the ohmic losses in the conductive earth. Zablocki did not come to

Table 1. Components of the electric and magnetic fields for various source geometries. Directions are in reference to a vector pointing from the measurement point to surface ground zero. The notations are as follows: $\mathrm{r}$ =radial, tan = tangential, $\mathrm{z}=$ vertical, $O=$ no field present, $X=$ field component present.

\section{Direction}

\begin{tabular}{lllllll}
\cline { 2 - 6 } Source geometry & $E_{\mathrm{z}}$ & $E_{\mathrm{r}}$ & $E_{\text {tan }}$ & $H_{\mathrm{z}}$ & $H_{\mathrm{r}}$ & $H_{\text {tan }}$ \\
\hline Vertical magnetic dipole & $\mathrm{O}$ & $\mathrm{O}$ & $\mathrm{X}$ & $\mathrm{X}$ & $\mathrm{X}$ & $\mathrm{O}$ \\
Horizontal magnetic dipole & $\mathrm{O}$ & $\mathrm{X}$ & $\mathrm{X}$ & $\mathrm{X}$ & $\mathrm{X}$ & $\mathrm{X}$ \\
Vertical electric dipole & $\mathrm{X}$ & $\mathrm{X}$ & $\mathrm{O}$ & $\mathrm{O}$ & $\mathrm{O}$ & $\mathrm{X}$ \\
Horizontal electric dipole & $\mathrm{X}$ & $\mathrm{X}$ & $\mathrm{X}$ & $\mathrm{X}$ & $\mathrm{X}$ & $\mathrm{X}$
\end{tabular}


any definite conclusions about the characteristics of the source for the tests he monitored: some had characteristics of vertical electric dipoles, and some had characteristics of magnetic dipoles oriented with the earth's magnetic field. He estimated a dipole electric source strength of $\sim 7 \times 10^{4} \mathrm{~A} \cdot \mathrm{m}$ for the Halite Program's Gnome event, detonated at a depth of $361 \mathrm{~m}$. In general, the initial signal spike had a rise time of about 8 to $12 \mathrm{~ms}$ with an overshoot lasting 0.5 to $1 \mathrm{~s}$. He found power spectrums for the overshoot interval to have frequencies of 4 to $8 \mathrm{~Hz}$, with considerable energy at $<2 \mathrm{~Hz}$.

Analyses by Malik et al.,12 of results from several underground nuclear explosions were also inconclusive. These researchers investigated the possibility that fields are being generated by a "seismic-electric" effect in which EM fields are generated when a seismic wave reaches the ground surface, and by an "electroseismic effect" in which a pressure wave impacts pore fluids carrying charged particles. Malick et al., think that their data support the seismic-electric source theory, but they cannot rule out the possibility of a magnetic dipole source. As an important side note, Malick et al., point out that Martner and Sparks ${ }^{13}$ observed electrical signals from small high-explosive charges detonated in boreholes.

Modern-day underground nuclear tests are often complicated physics experiments. They include large complex mechanical structures that are associated with the nuclear device and diagnostics. Hundreds of cables carry electrical data out of the hole to equipment located several hundred meters from surface ground zero. These conductors carry impulses created by the detonation fireball as well as diagnostic information. Many opportunities exist for radiated fields from the numerous conductors to be reflected and refracted by metal structures on the surface, which increases the complexity of the nearfield $\mathrm{EM}$ behavior.

Factors that can influence the nature of EM fields generated downhole by the device include the following: the design and shape of the canister, the location of the water table with respect to the working point, and the nature of the earth material surrounding the shot point. Other complications include the fact that we do not know if the source is characterized by a magnetic dipole, an electric dipole, or some combination, and we do not know how the source is oriented. Other fields can be generated by electroseismic or seismic-electric effects. With such complications, it is a daunting task to try to model or even to estimate a source strength for ELF radiation resulting from an underground nuclear test. Zablocki's estimate of source strength from the Gnome event, detonated in the Halite Program (see above), is about 1000 times smaller than that of a typical lightning bolt. This suggests that underground nuclear tests are not likely to be strong generators of ELF energy, but it does not answer the question as to whether we can detect underground tests by monitoring ELF signals.

Because of all the uncertainties involved in a theoretical approach to deciding whether ELF monitoring of underground tests is viable, we chose to carry out an experimental program to determine what can be measured rather than try to explain how the signals are generated.

The following sections of this report describe the equipment that we used to monitor ELF signals and the results of the measurements that we carried out during numerous underground nuclear tests at NTS and during one test (the Joint Verification Experiment) at the Soviet test site at Semipalatinsk.

\section{Equipment Used to Monitor ELF Signals}

\section{Data Acquisition and Storage}

A system to acquire and store ELF EM signals contains three basic parts:

- Sensors to convert changes in field strength to electrical signals.

- Pre-amplifiers, amplifiers, and filters to enhance the signals.

- Some type of storage system to store the signals for later analysis.

For this project, we used two different systems for analog-to-digital (A/D) conversion and storage.
One system, which the LLNL and UCLA teams both used, included an IBM AT personal computer for A/D conversion and storage on a hard disk. The system software was programmed by D. D. Sentman (UCLA) in the $C$ language. With this system, we could sample up to eight channels simultaneously at rates of $120 \mathrm{~Hz}$ or higher, depending on the number of channels sampled. In addition to the IBM AT, the monitoring system included a Kinemetrics Tracetime Model 468-DC satellite clock that locked to signals from the Geocentric Orbiting Environmental Satellite (GOES) for an accurate timing reference. 
The complete ELF measurement system that Sentman uses in his research and employed in his work for this project is described in detail in Refs. 14 and 15. The IBM AT system used by the LLNL team was identical to that used by Sentman except that it did not incorporate the signal-processing features that he uses to carry out real-time fast-Fourier transforms of the data.

Originally at LLNL, we could only handle the data acquired by the LLNL and UCLA computers in the time domain. Later in the project, we developed software at LLNL to convert the IBM AT data files to a form that could be transferred to the PRIME mainframe computer and later to a SUN workstation. With this capability, we could carry out signal-processing operations at LLNL using the Seismic Analysis Code (SAC) developed at LLNL for processing seismic data. The IBM AT-based system performed flawlessly, but it had one drawback in that the computer required ac power, which often was not available in remote locations. The advantage of this system was that the data could be examined shortly after it was acquired in the computer, making it easy to make on-site adjustments to the sensors, pre-amplifiers, and filters; therefore, minimizing local interference problems.

A second data acquisition system used by LLNL included Kinemetrics PDR-2 portable data recorders. The PDR-2 has been used for many years in the Seismology and Applied Geophysics Group at LLNL to acquire seismic data at remote field locations in the frequency range of 0 to $30 \mathrm{~Hz}$. The system runs on battery power and performs $\mathrm{A} / \mathrm{D}$ conversion and data sampling on up to six channcls at rates up to $200 \mathrm{~Hz}$, depending on the number of channels recorded. In a three-channel operation, up to $22 \mathrm{~min}$ of data could be stored on cassette tape, which could be later read into the PRIME mainframe computer or into a SUN workstation for data analysis. The system also had a built-in satellite clock, which locked onto signals from the GOES for the timing reference. A disadvantage of the PDR-2 system is that data quality is difficult to check while the system is in the field. The real-time signals can be monitored on an oscilloscope, but in this mode it is hard to assess the effects of local power-line noise or other sources of ELF interference.

\section{EM Field Sensors}

The task of measuring ELF signals is described in Ref. 14 as ". . . a problem of detecting a small signal superimposed on a large, noisy background, usually generated locally at adjacent frequencies." To measure magnetic field components, we must detect signals on the order of $1 \mathrm{pT}$ superimposed on the geomagnetic field of the earth, which is on the order of $5 \times 10^{7} \mathrm{pT}$. (The unit pT is a pico-Tesla, or 10.12 Tesla. One pT equals $0.001 \mathrm{nT}$, or $0.001 \gamma$ which equals $10^{-8}$ gauss.) The fair-weather vertical electric field of the earth is $\sim 150 \mathrm{~V} / \mathrm{m}$ at the earth's surface ${ }^{14}$; typical ELF fields superimposed on this are $\sim 0.1 \mathrm{mV} / \mathrm{m}$ or less. Therefore, the ELF sensors must be able to detect electrical and magnetic ELF signals that have very low field strength and noise characteristics.

The magnetic sensors used by LLNL for these ELF measurements were made by Electromagnetic Instruments, Inc. (EMI) in El Cerrito, Calif,, and were based on a design similar to that described by Morrison et al. ${ }^{16}$ These sensors, referred to as model BF-5, consisted of a coil with a high $\mu$-metal core with a carefully matched built-in amplifier. They were $<0.5 \mathrm{~m}$ long, $\sim 2.5 \mathrm{~cm}$ diam, and $<1 \mathrm{~kg}$ wt. The coil and its amplifier were sealed in a waterproof case, and the signal and power were conveyed over a short cable to a small battery-and-cable junction box. The sensors were tuned to $8 \mathrm{~Hz}$ and had, at that frequency, an intrinsic sensitivity of 1.9 $\mathrm{mV} / \gamma$. The noise at $8 \mathrm{~Hz}$ was $\sim 20 \mu \gamma / \mathrm{Hz}^{1 / 2}$. Factory calibration of the sensor BF-5-LLL revealed a response of $\sim 100 \mathrm{mV} / \mathrm{nT}$ that is flat between 6 and $15 \mathrm{~Hz}$ with a gradual dropoff at higher and lower frequencies. This is equivalent to $10^{-4} \mathrm{~V} / \mathrm{pT}$, or $10^{4}$ $\mathrm{pT} / \mathrm{V}$, of electrical signal out of the magnetometeramplifier unit. The magnetometers used by Sentman are older SQUID coils that are physically much larger and heavier, but have essentially the same signal detection qualities as the EMI coils.

Figure 4 shows an EMI magnetometer being installed in the field at NTS. Because it has a built-in pre-amplifier, we only needed $\sim 30 \mathrm{db}$ of additional amplification before sending the magnetic signals to the PDR-2, which has an internal gain of 8 . The total system gain for the magnetic signals is $\sim 250$.

In the measurements for this project, we always used the geographic north and east reference directions for the horizontal magnetometer placement. The magnetometers were buried 15 to $30 \mathrm{~cm}$ deep in a shallow trench. The soil was smoothed over the surface to minimize wind vibration. The vertical magnetometer was placed in a vertical hole with only the signal and power cables coming out at the surface. Soil or sand was packed around the magnetometer to prevent sideways movement. The 


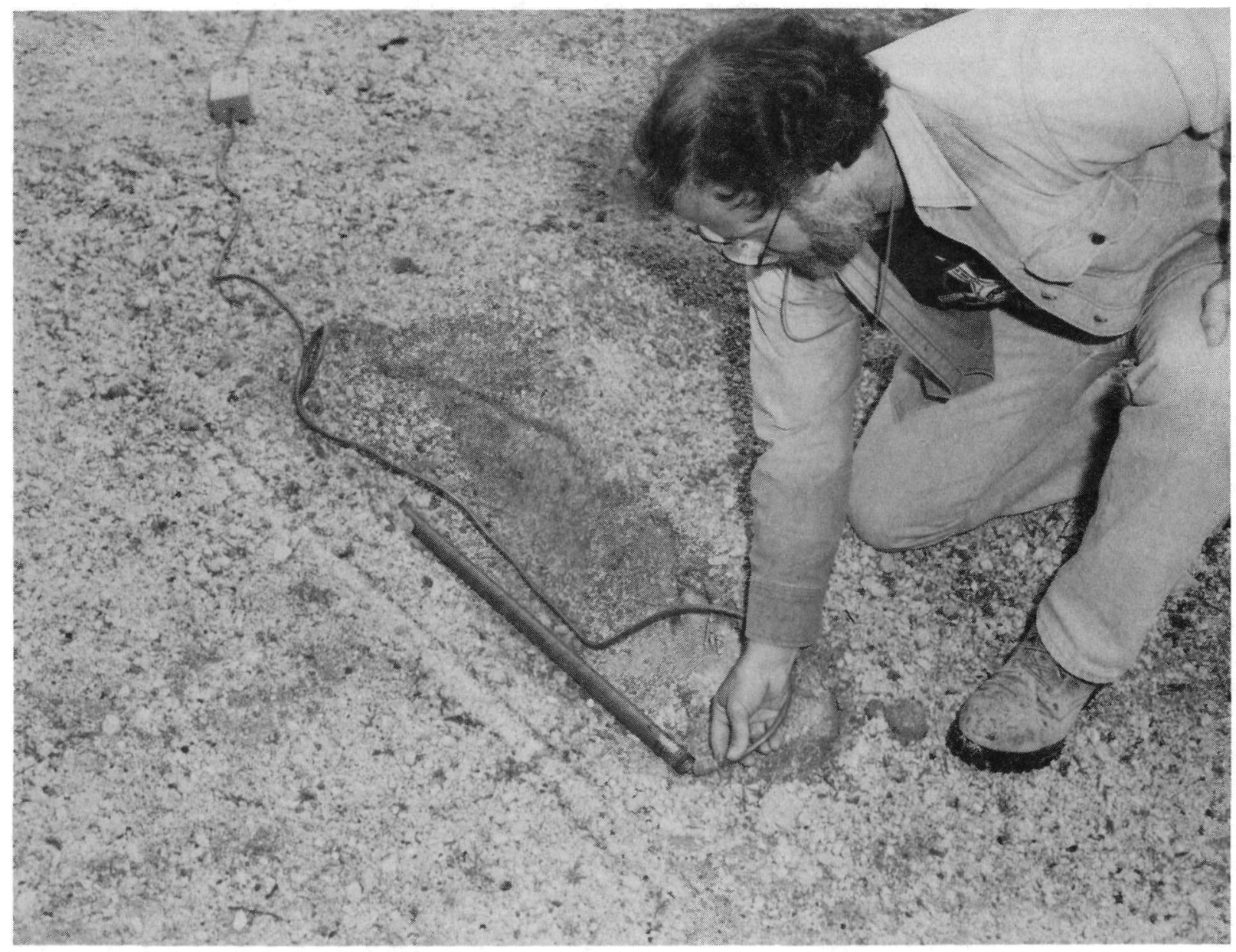

Figure 4. The author installing a magnetometer in a shallow trench on Pahute Mesa at NTS.

magnetometers were located as far from small plants and other sources of vibrational noise as possible.

The electrical sensors consisted of an antenna (essentially a large capacitor) to measure the vertical electric field and of north- and east-directed telluric lines to measure the horizontal electric field. The vertical electric sensor was a steel ball, $\sim 30 \mathrm{~cm}$ in diameter, mounted on top of a section of PVC pipe about $2 \mathrm{~m}$ long and $15 \mathrm{~cm}$ diam. The lower end of the PVC pipe went through a hole centered in 4 aluminum ground plates, each $\sim 2 \mathrm{~m}^{2}$ (see Fig. 5). The lower $0.5 \mathrm{~m}$ of PVC pipe was solidly tamped into the ground to provide maximum stability for the entire structure. The edges of the aluminum plates were covered with soil to minimize wind vibration. The signal lead from the steel ball was carried via a shielded-cable inside the PVC pipe and was buried under the ground reference plate until it came to the surface for connection with a pre- amplifier. The whole design was a compromise to obtain maximum signal strength (which depends on the height of the ball above the ground reference) with maximum mechanical stability.

The sensor is extremely sensitive to wind, to motion of nearby plants, and even to clouds passing overhead. A vertical movement of $1 \mathrm{~mm}$ of the height of the ball with respect to the reference plate can create a signal of $\sim 0.15 \mathrm{mV} / \mathrm{m}$, which is on the order of most ELF signals of interest.14 Much of the local vibration and wind noise affecting the antenna occur at frequencies below $4 \mathrm{~Hz}$; therefore high-pass filtering is usually used to enhance the Schumann resonance detection capabilities. If a moderate-to-strong wind is blowing or if there is local precipitation, the vertical electric sensor will, in most cases, be saturated with useless noise.

The horizontal electric field sensors consisted of telluric lines fabricated from $\sim 100 \mathrm{~m}$ of wire, which was laid on the ground surface and was 


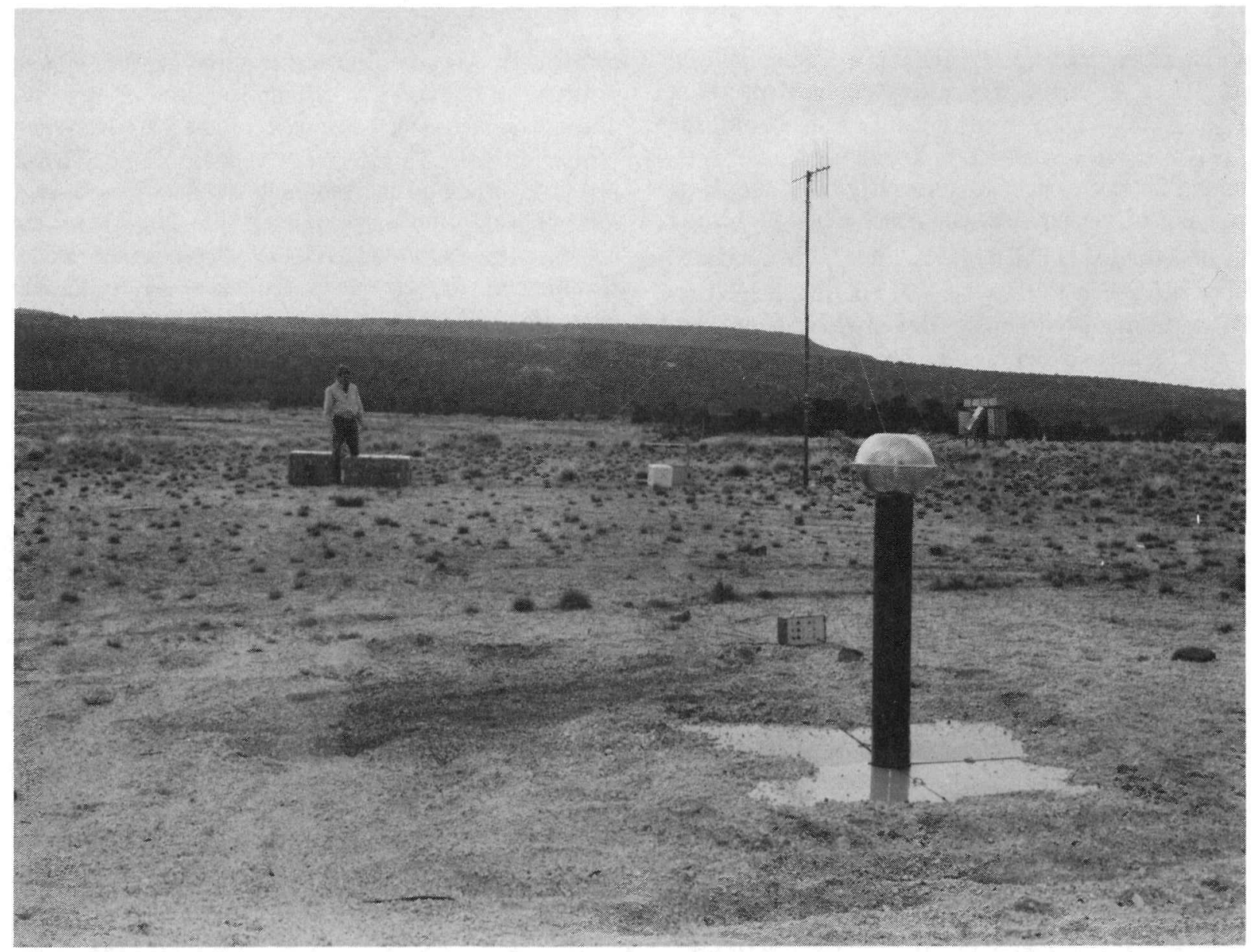

Figure 5. A view of a typical deployment of an ELF station, with PDR-2 data-acquisition gear, on Pahute Mesa, NTS. The technician is standing next to the PDR-2 data acquisition and amplifier boxes. The ELF vertical electric antenna is in the foreground with the small electric-channel preamplifier box immediately behind it. In the background, to the left of the high-frequency antenna, are boxes containing high-frequency transponders to remotely trigger the PDR-2.

connected to buried porous pots. These porous pots, routinely used in spontaneous potential geophysical measurements, were small cylinders, each with a porous ceramic at one end. The cylinders were filled with a supersaturated solution of cupric sulfate; each was sealed at the top with a plug containing a copper electrode that extended into the solution. The purpose of these pots was to provide a good electrical connection to the ground and to avoid polarization problems that can occur when metal electrodes are placed directly into the soil.

Soil and rocks were placed on the telluric wires to minimize vibration, which can create a signal when the wire moves through the geomagnetic field. We tried to lay the horizontal lines over flat terrain because the vertical electric field has $~ 1000$ times the strength of the horizontal field, so we wanted to avoid picking up any vertical components on the telluric lines.

\section{Pre-Amplificrs}

Because of the high impedance of the vertical elcctric antenna and the low noise requirements of the ELF measurement system, we used operational amplifier integrated-circuit pre-amplifiers with input impedances of $10^{9} \Omega$. The LLNL pre-amplifier design was quite similar to that of the UCLA team ${ }^{14}$ and was also used for horizontal electric signals, even though the telluric lines have relatively 
low input impedance. Pre-amplifier gains ranged from 10 to 200 , with a total system gain of about 100 to 1000 . The high input impedance of the system made it susceptible to oscillations and crosscoupling between channels through ground loops, so the layout of the physical components and grounding was important. ${ }^{14}$ The pre-amplifiers also contained bandpass filtering to pass 4 to $30 \mathrm{~Hz}$; this helped reduce the noise from wind and vibration at the lowfrequency end and the $60-\mathrm{Hz}$ power-line interference at the high-frequency end. In some cases in which local power line interference was a problem, we added $60-\mathrm{Hz}$ notch filters at the pre-amplifiers. With the IBM AT, we could also use a $120-\mathrm{Hz}$ sampling rate that further reduced the $60-\mathrm{Hz}$ interference with aliasing.

\section{Calibration}

We decided that precise calibration of the ELF measurement system was not necessary for this project because we were mainly concerned with signal detection. However, we were able to estimate field strengths by using sensor calibrations and our knowledge of system gains.

The vertical electric antenna was calibrated by putting it beneath a set of aluminum plates that were identical to the ground reference plates. In this configuration, the steel ball is placed between plates of a large capacitor. A known voltage is applied across the plates. The field strength at the ball is easily determined by measuring the output from the antenna and pre-amplifier versus the frequency of the applied signal. A typical equivalent output of the antenna was $-0.43 \mathrm{~V}$ per $\mathrm{V} / \mathrm{m}$ of the applied electric field strength. For a system gain of 400 , a $10 \mu \mathrm{V} / \mathrm{m}$ field strength thus produced a 1.7 . $\mathrm{V}$ signal at the data-recording system. For the horizontal electric field measurements, the 100 - $\mathrm{m}$-long telluric line produced $0.1 \mathrm{mV}$ for each $\mu \mathrm{V} / \mathrm{m}$ of applied field. With a gain of 200, a $50-\mu \mathrm{V} / \mathrm{m}$ field strength produced an output signal of $1.0 \mathrm{~V}$.

As we noted above, the magnetometers were factory-calibrated to produce $10^{-4} \mathrm{~V} / \mathrm{pT}$ of field strength change. Typically, we used total gains of 253; thus, a signal of 39.5 pT produced an output signal of $1.0 \mathrm{~V}$. Various gains were used with individual systems at varying times, making the calibration factors slightly different for each deployment.

These calibration estimates do not take into account the system frequency response nor the timing and phase delays inherent in the amplifiers and filters. A complete description of the system response of the UCLA system is given in Ref. 14. The overall frequency response of the UCLA system is roughly 4 to $30 \mathrm{~Hz}$. The response of the PDR-2 system depends on the sampling frequency used: in a three-channel mode, sampling at $200 \mathrm{~Hz}$, the response is -4 to $25 \mathrm{~Hz}$; in a 6-channel mode, sampling at $100 \mathrm{~Hz}$, the response is -4 to $15 \mathrm{~Hz}$. Note that the low-frequency cutoff is due to the high pass filters designed into the pre-amplifiers. A comparison of the frequency response spectra of the three system arrangements is shown in Fig. 6.

\section{Field Deployment and Triggering}

The portable PDR-2-based data-acquisition system was used in the initial stages of the project to acquire data at NTS and near LLNL. When the recording equipment was not deployed in forward areas, we simply listened to the countdown for a nuclear test and turned on the equipment at the proper time. Later, when the equipment was set up within several kilometers of ground zero at NTS and when we could not be present at detonation time, we employed a "tone barrel" trigger signal, supplied by EG\&G personnel, to remotely start the PDR-2 dataacquisition unit. The equipment was generally programmed to turn on $1.5 \mathrm{~min}$ before detonation and turn off $\sim 20 \mathrm{~min}$ later. Figure 5 shows a complete portable ELF field station (the buried magnetometers and telluric lines are not visible), including the vertical electric field sensor, PDR-2 data recorder and amplifier, tone barrel receiver, and high-frequency antenna. These stations were deployed at numerous NTS locations (Pahute Mesa, Buckboard Mesa, Yucca Flat, Gold Meadows, and Frenchman Flat ) as described in the next section.

The IBM AT data-acquisition system was first deployed by Sentman at a permanent station at Table Mountain (northeast of Los Angeles). This station recorded the vertical electric field and the two horizontal magnetic fields. Data were obtained by this station for many of the NTS events monitored for this project. In September 1986, a similar system was set up at NTS Area 20 Camp at Pahute Mesa to record all three orthogonal components of the electric and magnetic fields. After monitoring several events at this station, the equipment was moved to Area 8, near the general services area, for the remainder of the project. During the late summer of 1987 , Los Alamos National Laboratory funded a third IBM AT-based data-acquisition system, which Sentman established in the southern part of Frenchman Flat. Thus, for various underground tests, from one 


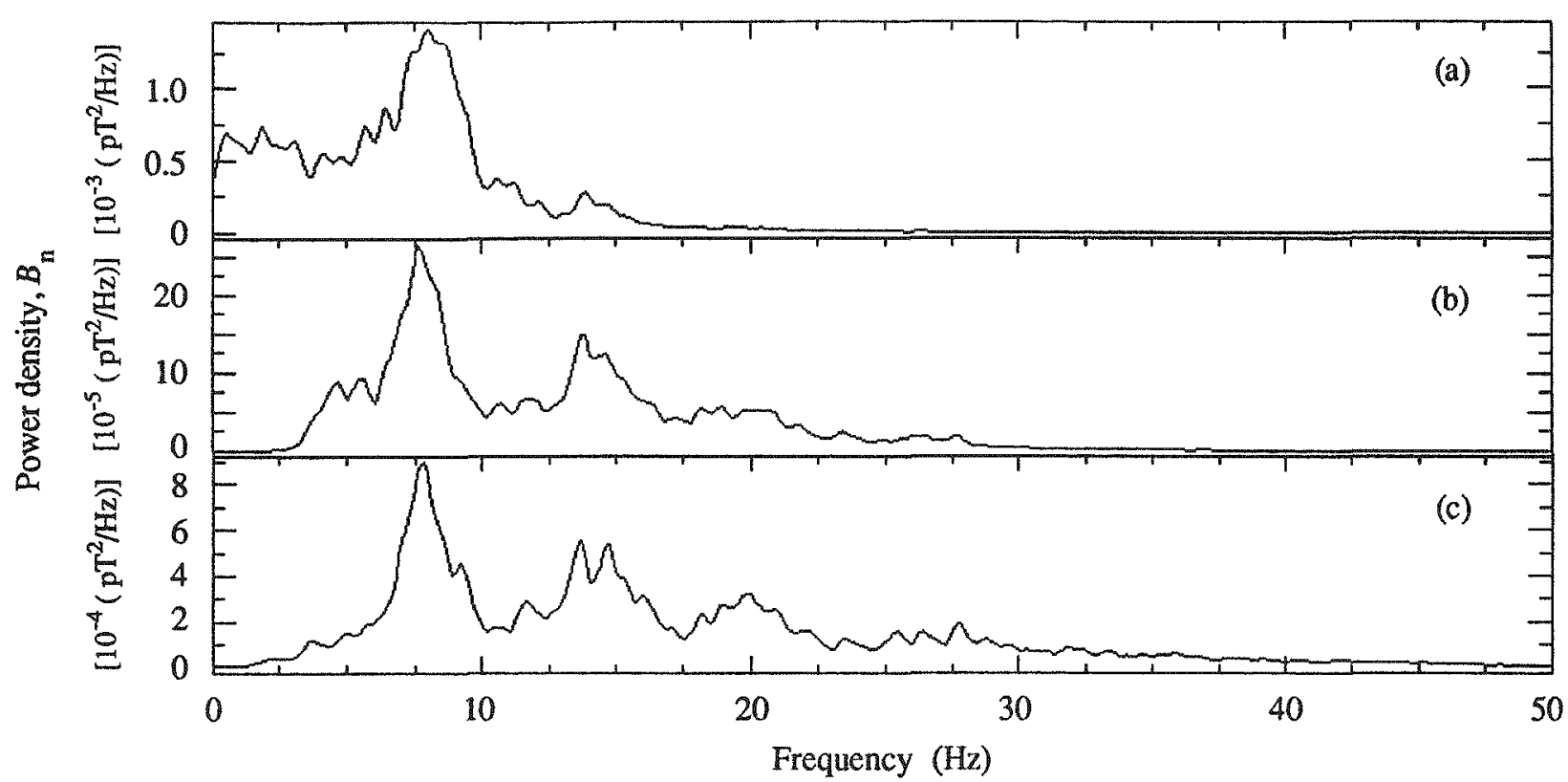

Figure 6. Power-density spectra, comparing the bandpass characteristics of various data-acquisition systems. All the spectra are for $150 \mathrm{~s}$ of north magnetic field component data. (a) Spectrum from the PDR-2 sampling at $100 \mathrm{~Hz}$. (b) Spectrum from the PDR-2 sampling at $200 \mathrm{~Hz}$. (c) Spectrum from the IBM AT system sampling at $120 \mathrm{~Hz}$.

to three ELF stations were deployed by the UCLA team.

An additional IBM AT station was established near LLNL during the spring of 1987 . Sensors to detect the vertical electric field and the two horizontal magnetic fields were installed near the CPP seismometer station (about 2 miles northeast of the LLNL site). The ELF analog signals were transmitted to LLNL via existing seismic station of telemetry; the IBM AT received the signals, performed the $A / D$ conversion, and stored the data. This system was in operation almost continuously during 1988, and many NTS underground tests as well as a few Soviet tests were monitored during that time.

\section{ELF Monitoring Results from Underground Nuclear Tests}

\section{Summary of Results}

We monitored 29 underground nuclear tests for ELF EM signals: 25 were tests at NTS and 4 were tests in the Soviet Union. Because it is impractical to discuss the results of these tests in detail, I summarize them in tables and discuss the details of several representative tests, pointing out the most important aspects of the data.

All of the underground nuclear tests that, to my knowledge, were monitored for ELF EM emissions by LLNL from FY 1986 through FY 1988 are listed in Table 2. The first three events (Roquefort, Kinibito, and Goldstone) were monitored by H. M. Buettner (LLNL), who reported the resulis in an
LLNL internal memorandum. For Roquefort and Goldstone, Buettner deployed a three-component $\left(E_{\mathrm{z}}, B_{\mathrm{n}}\right.$, and $\left.B_{\mathrm{e}}\right)$ ELF station at NTS that was located $30 \mathrm{~km}$ or more from ground zero (exact station locations were not given in Buettner's memorandum). For Kinibito, he deployed a three-component station a few kilometers south of LLNL. In all three tests, no ELF signals were detected at detonation time that could be attributed to an underground test. The $E_{\mathrm{z}}$ signals obtained for these tests are of poor quality because of wind noise. The magnetic field measurements are of fairly good quality, and the sensitivity is comparable to that obtained in later measurements. 
Table 2. Time and date of detonation of underground nuclear tests monitored for ELF EM signals.

\begin{tabular}{|c|c|c|}
\hline Event name & Date & $\begin{array}{l}\text { Time } \\
(\mathrm{UT})^{\mathrm{a}}\end{array}$ \\
\hline Roquefort & $10-16-85$ & $21: 35: 00$ \\
\hline Kinibito & $12-06-85$ & $15: 14: 00$ \\
\hline Goldstone & $12-28-85$ & 19:01:00 \\
\hline Glencoe & $3-22-86$ & $16: 15: 00$ \\
\hline Jefferson & $4-22-86$ & $14: 30: 00$ \\
\hline Panamint & $5-21-86$ & $13: 59: 00$ \\
\hline Tajo & $6-05-86$ & $15: 04: 00$ \\
\hline Darwin & $6-25-86$ & $20: 27: 45$ \\
\hline Cybar & $7-17-86$ & $21: 00: 00$ \\
\hline Labquark & $9-30-86$ & $22: 30: 00$ \\
\hline Belmont & $10-16-86$ & $19: 25: 00$ \\
\hline Delamar & $4-18-87$ & $06: 40: 00$ \\
\hline Presidio & $4-22-87$ & $22: 00: 00$ \\
\hline Hardin & $4-30-87$ & $13: 30: 00$ \\
\hline USSR & $5-06-88$ & b \\
\hline USSR & $6-06-87$ & $b$ \\
\hline USSR & $7-17-87$ & b \\
\hline Midland & $7-16-87$ & $19: 00: 00$ \\
\hline Tahoka & $8-13-87$ & $14: 00: 00$ \\
\hline Lockney & $9-24-87$ & $15: 00: 00$ \\
\hline Borate & $10-23-87$ & $16: 00: 00$ \\
\hline Waco & $12-01-87$ & $16: 30: 00$ \\
\hline Mission Cyber & $12-02-87$ & $16: 30: 00$ \\
\hline Kemville & $2-05-88$ & $18: 10: 00$ \\
\hline Shellbourne & $5-13-88$ & $15: 35: 00$ \\
\hline Comstock & $6-02-88$ & $13: 00: 00$ \\
\hline Alamo & $7-07-88$ & $15: 05: 30$ \\
\hline Kearsarge & $8-17-88$ & $17: 00: 00$ \\
\hline JVE, USSR & $9-14-88$ & $04: 00: 00$ \\
\hline
\end{tabular}

\footnotetext{
${ }^{a} \mathrm{UT}$ is universal time.

These presumed Soviet Union tests were inferred from NORESS data; the origin time is estimated from seismic arrival phases.
}

The next series of NTS tests monitored for ELF were in FY 1986. They were Glencoe, Jefferson, Panamint, Tajo, Darwin, and Cybar and were monitored under the direction of P. W. Rodgers (LLNL). For these tests, the ELF signals were measured at NTS several tens of kilometers away from ground zero and at the east operation point near the advanced test accelerator at Site 300 southeast of LLNL. Again, no ELF signals clearly related to the test detonation could be seen in the data.
Thus, by late FY 1986, obvious signals had not been detected, but we felt that it was possible that a signal was present in the data, but we just did not know what it looked like or if the signal-to-noise ratio could be enhanced by signal processing. Rodgers decided to move the ELF measurement stations closer to the source to increase our chances of detecting a signal from a test and, therefore, possibly characterizing the nature of the source signal. He also added the capability to measure all three components of both the magnetic and electric fields (vertical electric $E_{\mathrm{z}}$, north electric $E_{\mathrm{n}}$, east electric $E_{\mathrm{e}}$, vertical magnetic $B_{\mathrm{z}}$, north magnetic $B_{\mathrm{n}}$, and east magnetic $B_{\mathrm{e}}$ ).

The data, obtained at NTS in FY 1987 and FY 1988, consist of measurements from ELF stations located both distant from and near to the tests. Table 3 shows the results of these ELF monitoring stations. The number of observation sites varied for different tests, depending on whether the team from UCLA was available and whether the equipment at each site was operating properly.

In the spring of 1987, the LLNL team installed a permanent station at the CPP site near LLNL (sce footnotes to Table 3). This station collected three-component $\left(E_{\mathrm{z}}, B_{\mathrm{n}}\right.$, and $\left.B_{\mathrm{e}}\right)$ ELF signals, which were sent via existing telemetry to LLNL where they were processed and stored on the IBM AT data-acquisition system. The storage capability of the computer allowed us to store up to 24 hr of three-component ELF data so that, if the Norwegian seismic array (NORESS) detected an inferred underground test in the Soviet Union and if we were notified in time, we could look back into our data to see if it showed an ELF signal related to the test. This was a difficult process; we did not know the exact origin time of the event, and we were not sure what to look for in the ELF data. While the CPP station was operating, LLNL personnel notified us about three inferred Soviet Union tests in time for us to review our stored data (these tests are listed in Table 2); the data did not show an ELF signal obviously related to any of these tests. There are also two NTS tests listed in Table 2 (not listed in Table 3) that were monitored only by the CPP station. These tests are Presidio and Midland; in both cases, no ELF signals were detected at detonation time.

Table 3 lists the name of the tests monitored during FY 1987 and FY 1988, the names of the ELF monitoring stations (the locations of the sites are noted in the bottom half of the table), and the distances from surface ground zero. (The Nevada coordinates for the hole locations of each test are on file with the LLNL Containment Program.) This table 
Table 3. Underground nuclear tests monitored in FY 1987 and FY 1988. Results of ELF EM monitoring are divided into locations at which a distinct signal, clearly related to the detonation of the device, were observed on at least one component of the ELF EM signals monitored and into locations at which no signals were defected.

$\begin{array}{lll}\text { Event } & \text { Signal detected }(\mathrm{km}) \quad \text { Signal not detected }(\mathrm{km})\end{array}$

Labquark

Belmont

Delamar

Hardin

Tahoka

Lockney

Borate

Waco

Mission Cyber

Kernville

Shellbourne

Comstock

Alamo

Kearsarge

JVE USSR
$\mathrm{A} 20(7.4), \mathrm{EG}(6.2), 18 \mathrm{R}(9.5)$

A20 (4.9), 19X (5.0)

A7 (5.5)

HR (7.9)

A8 (3.1)

HR (8.8)

A8 (5.09)

EG (5.0)
GM (11.6), A19 (8.4)

18R (13.7), 19X (12.7), CPP (485)

18R (11), CPP (485)

CPP (485)

EG (11.7), FF (73.9), TM (420), CPP (485)

FF (48), TM (420), CPP (485)

GM (31.7), FF (32.2)

GM (5.5), FF (60.1)

$18 \mathrm{R}(20), \mathrm{A} 8(37.2), \mathrm{FF}(86.6), \mathrm{TM}(420)$, CPP (485)

Al (19.2), FF (46.2), CPP (485)

$18 \mathrm{R}(13.8), \mathrm{A} 8(32.5)$, FF (80.8)

$\mathrm{CPP}(485)$

A8 (23.7), FF (74.4), CPP (485), FB (3800)

$\mathrm{A} 8(10,300), \mathrm{FF}(10,300), \mathrm{FB}(6600)$

Station notations and locations (Nevada coordinates in meters):

GM at NTS, Gold Meadows (N275021, E192710);

EG at NTS, Egmont (N279441, E174529);

A19 at NTS, Area 19 (N285384, E176205);

A20 at NTS, Area 20 (N280355, E174529);

18R at NTS, Area 18 (N264673, E172090);

A1 at NTS, Area 1 (N252222, E189738);

$19 X$ at NTS, Area 19 (N275372, E178994);

A7 at NTS, Area 7 (N261305, E210406);

HR at NTS, Hardin (N275814, E170871);

A8 at NTS, Area 8 (N268255, E203195);

FF at NTS, Frenchman Flat (N230124, E237073);

CPP near LLNL, co-located with the CPP seismic station, $\sim 2 \mathrm{~km}$ northeast of LLNL;

TM at the UCLA Table Mountain site in the San Gabriel Mountains, northeast of Los Angeles;

$\mathrm{FB}$ at the NOAA site $\sim 24 \mathrm{~km}$ northeast of Fairbanks.

shows that the ELF signals were generally detected at stations located $<10 \mathrm{~km}$ from surface ground zero. Exceptions to this are for the A19 station during the Labquark test and for the GM station during the Mission Cyber test. Mission Cyber was a low-yield tunnel test. In all cases, where monitoring stations were located farther than $10 \mathrm{~km}$ from ground zero, no ELF signals were measured that were associated with the test. In the next three sections, I will discuss the details of the NTS Hardin and Kernville tests and the Joint Verification Experiment (JVE) tests at NTS (the Kearsarge test) and at the Shagan River Site in the Semipalatinsk region in the Soviet Union (the Shagan test). 


\section{The Hardin Test}

The Hardin test was the second underground nuclear test for which we detected ELF EM signals. Three ELF measurement stations were located on Pahute Mesa, NTS; two $\sim 5 \mathrm{~km}$ away from ground zero and one $\sim 11 \mathrm{~km}$ away from ground zero (see Table 3). The CPP three-component station near LLNL was also in operation for this test. Stations $18 \mathrm{R}$ and 19X were six-component stations using Kinemetrics' PDR-2 data recorders located south and east, respectively, of ground zero. Station A20 (located east northeast of ground zero near the trailers at Area $20 \mathrm{Camp)}$ was a six-component station fielded by the UCLA team using an IBM AT for data recording. The PDR-2 data recorders were started $90 \mathrm{~s}$ before detonation time $\left(T_{0}\right)$ with a highfrequency radio remote control. The UCLA station collected data continuously and stored $8 \mathrm{hr}$ of data before overwriting; Sentman had to be sure to get to the station in the forward area within $8 \mathrm{hr}$ of detonation time in order to save the data covering the few minutes before and after $T_{0}$.

The data from each component of the four stations are shown in Figs. 7-12; these plots show $5 \mathrm{~s}$ of data before $T_{0}$ and $4 \mathrm{~s}$ of data after $T_{0}$. Strong signals at $T_{0}$ are seen in all six components of the A20 station and in all but the $E_{\mathrm{z}}$ component at the 19X station. No signals are seen at the 18R and CPP stations at $T_{0}$, except for a possible signal in $B_{\mathrm{n}}$ at $18 \mathrm{R}$ (see Fig. 7). The strong signals seen $\sim 1.5 \mathrm{~s}$ after $T_{0}$ at $\mathrm{A} 20$ and $19 \mathrm{X}$ and $\sim 3 \mathrm{~s}$ after $T_{0}$ at $18 \mathrm{R}$ are caused by mechanical perturbation of the sensors by the arriving surface ground motion. The data for the $19 \mathrm{X}$ and $18 \mathrm{R}$ stations in Figs. $7-12$ were calibrated using the procedure described earlier in the "Calibration" section of this report. Data from the A20 station and the CPP station were calibrated for Figs. 7-12 by adjusting the magnitude of the background noise to be the same as that for stations $19 \mathrm{X}$ and 18R; thus, the amplitudes of data for $\mathrm{A} 20$ and CPP are almost correct but could be off by a factor of 2 or more. The A20 station also has more accurate frequency resolution because it has a bandpass about twice as wide $(-4$ to $30 \mathrm{~Hz})$ as the other three stations $(-4$ to $15 \mathrm{~Hz})$. The vertical electric data at stations $19 \mathrm{X}$ and CPP are severely degraded by local noise caused by wind.

The $T_{0}$ signals for the $B_{\mathrm{n}}$ and $B_{\mathrm{e}}$ components at $19 X$ and $A 20$ are roughly comparable in magnitude, although there are differences in their time signatures. The $B_{\mathrm{Z}}$ signal at $\mathrm{A} 20$ secms to be much larger than that at $19 \mathrm{X}$, although the data at the A20 station contains components of high frequency noise at $\sim 30 \mathrm{~Hz}$; this noise may have caused an error in the calibration of the signal magnitudes. The electric field data (Figs. 10-12) show even greater differences in magnitude between stations A20 and 19X; these differences may result from inaccuracies in magnitude calibration and from differences in bandpass characteristics among the data-recording systems.

Figure 13 compares the ELF-background frequency response of the $B_{\mathrm{n}}$ component $(90 \mathrm{~s}$ of data prior to $T_{0}$ ) for the three Pahute Mesa stations. The wider bandpass of the A20 station is evident because the second Schumann peak at $\sim 14 \mathrm{~Hz}$ is easily resolved, whereas the second peak is not prominent for the $18 \mathrm{R}$ and 19X stations. The difference in the bandpass for the two data collection systems is due to a limitation in the PDR-2 data recorders. The PDR-2 can sample at a maximum of $100 \mathrm{~Hz}$ when six channels are recorded. This limits the theoretical bandpass to 0 to $25 \mathrm{~Hz}$; however, in practice the limit is 0 to $15 \mathrm{~Hz}$. This limitation can be overcome (as was done for tests after Borate) by using two PDR2 recorders at each site and by recording three channels on each sampling at $200 \mathrm{~Hz}$; this technique effectively doubles the bandpass.

A high correlation exists between the horizontal magnetic ELF background signals for different stations widely separated from each other. This is especially true for the $B_{\mathrm{n}}$ component. As mentioned earlier in the "Natural and Man-Made Sources of ELF Signals" section of this report, the correlation of $B_{\mathrm{n}}$ can be used to check the relative time references between ELF stations. Even though the ELF stations use the GOES time reference, differences in relative timing between stations on the order of a few milliseconds can remain because of electronic delays in filters and amplifiers. In Fig. 14(a), the timing of the $B_{\mathrm{n}}$ component for stations A20, 19X, 18R, and CPP has been adjusted so that the strong $Q$ burst occurs at the same time at all four stations. When this is adjustment is made, other $Q$-bursts can be located in the data at other times that also match exactly in time; therefore, verifying this technique. The high correlation between the stations is further confirmed in Fig. 14(b), which shows the spectral coherence of $90 \mathrm{~s}$ of pre- $T_{0} \quad B_{\mathrm{n}}$ data between A20 and $19 \mathrm{X}$ and between $18 \mathrm{R}$ and $19 \mathrm{X}$. The plot shows that $80 \%$ of the energy between $\sim 2$ and $12 \mathrm{~Hz}$ is coherent.

When I originally looked at the data from the Hardin event, I noticed several bursts of energy in the magnetic components of the signals from the 


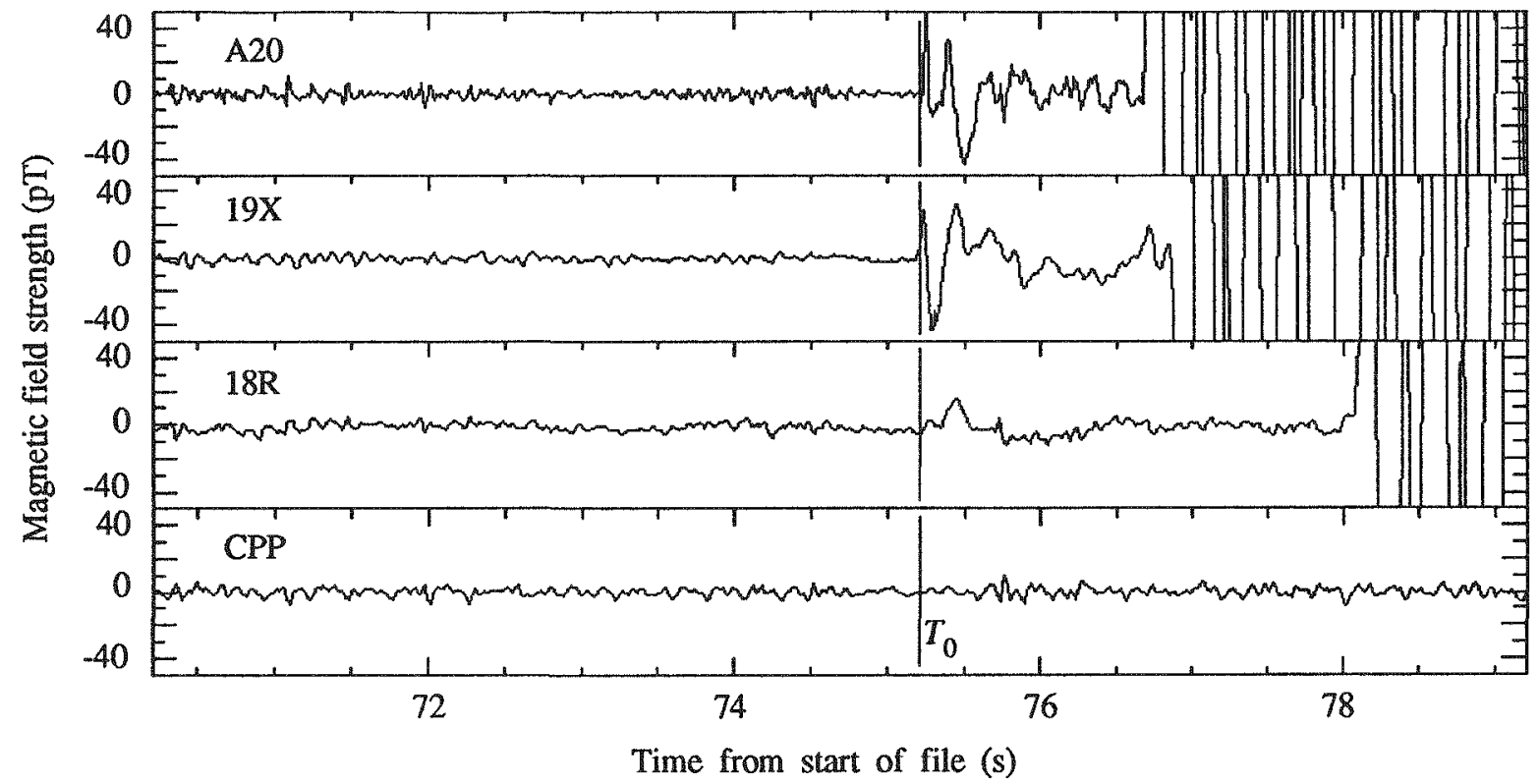

Figure 7. North magnetic $B_{\mathrm{n}}$ component of the ELF data from four sites, taken during the Hardin test. The detonation time is denoted by the vertical line marked $T_{0}$. Note the arrival of the surface ground motion at the magnetometer after $T_{0}$ at the $\mathrm{A} 20,19 \mathrm{X}$, and $18 \mathrm{R}$ sites.

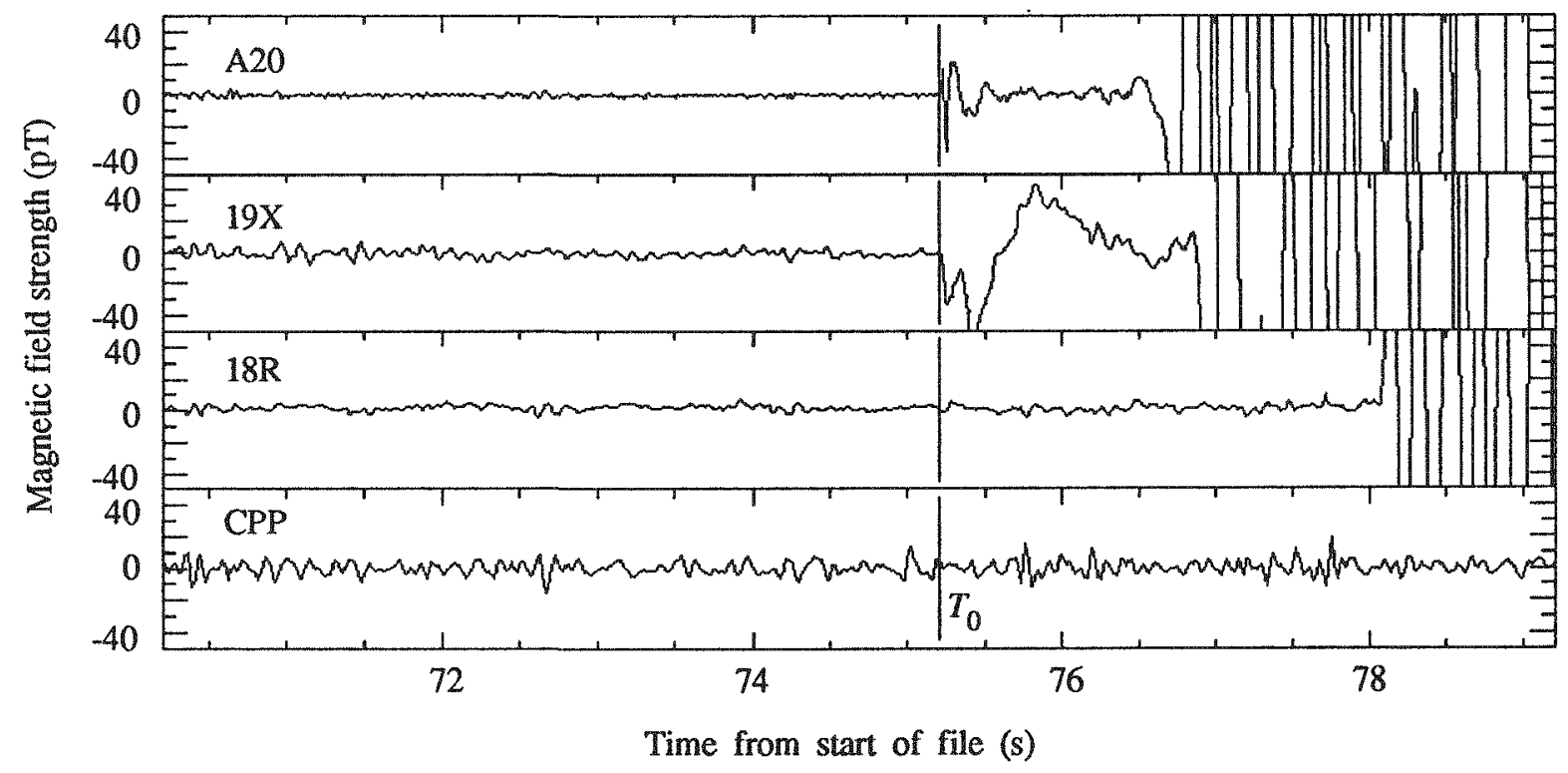

Figure 8. East magnetic $B_{\mathrm{e}}$ component of the ELF data from four sites, taken during the Hardin test. The detonation time is denoted by the vertical line marked $T_{0}$. Note the arrival of the surface ground motion at the magnetometer after $T_{0}$ at the $\mathrm{A} 20,19 \mathrm{X}$, and $18 \mathrm{R}$ sites. 


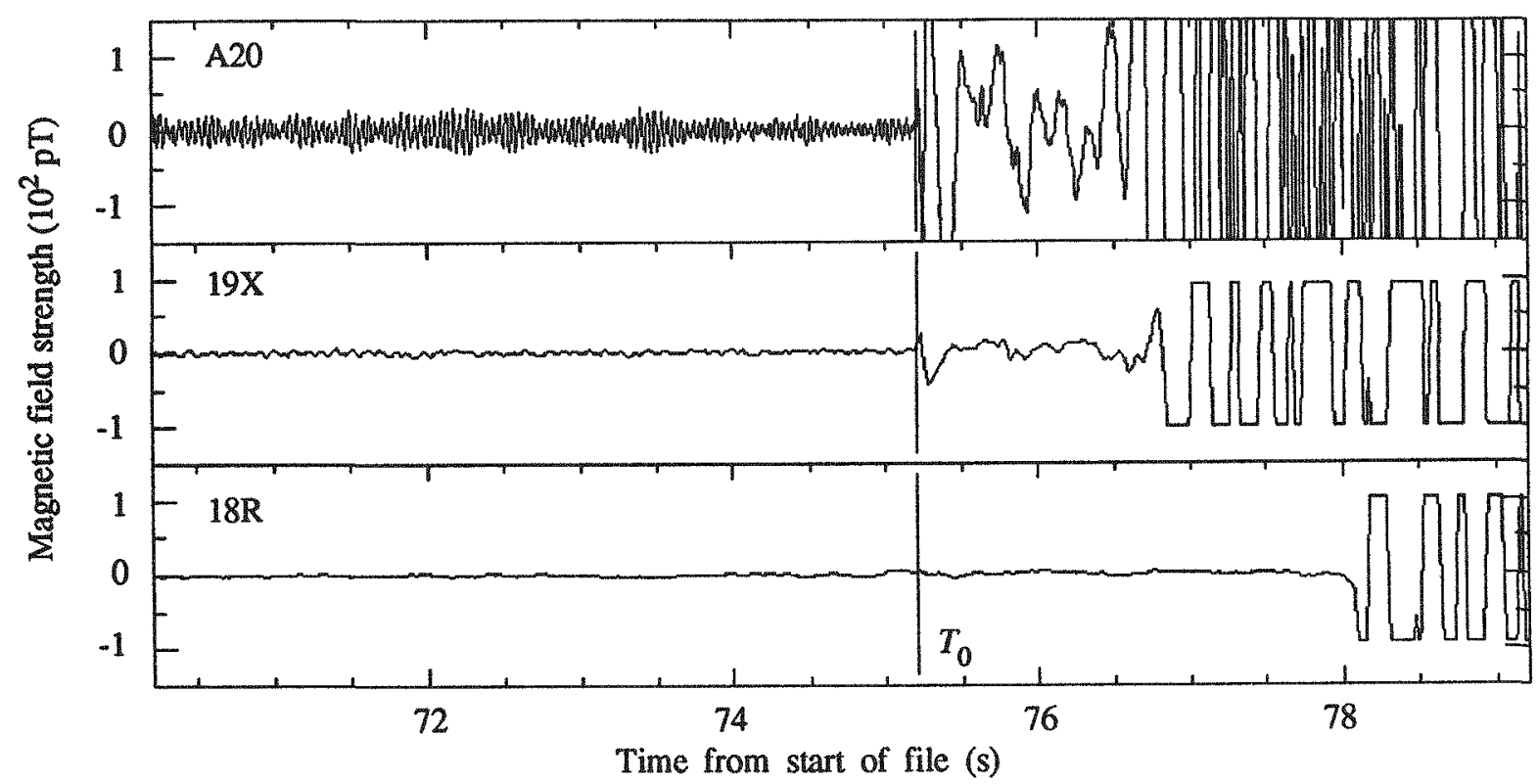

Figure 9. Vertical magnetic $B_{\mathrm{z}}$ component of the ELF data from three sites, taken during the Hardin test. The detonation time is denoted by the vertical line marked $T_{0}$. Note the arrival of the surface ground motion at the magnetometer.

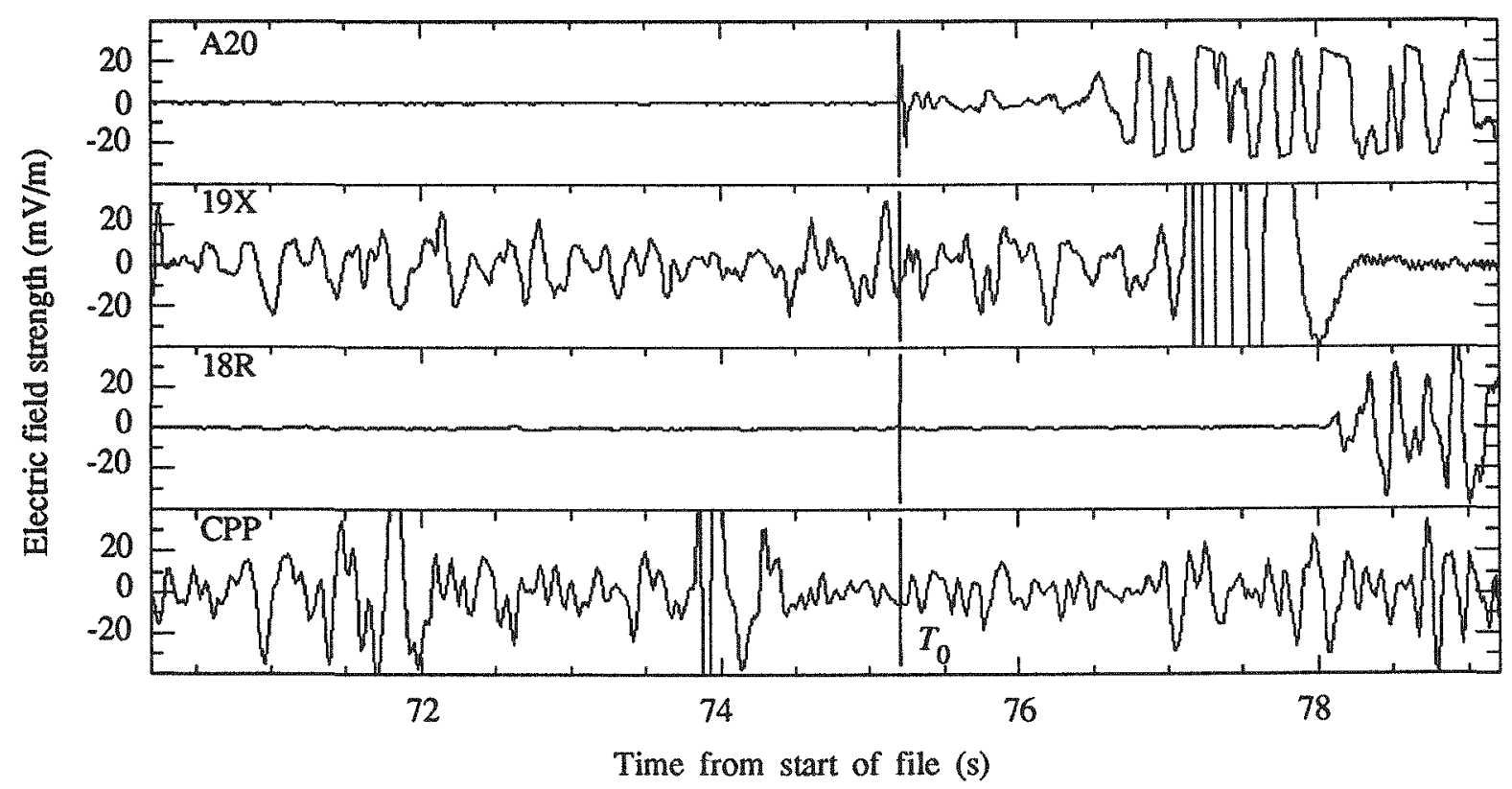

Figure 10. Vertical electric $E_{\mathrm{Z}}$ component of the ELF data from four sites, taken during the Hardin test. The detonation time is denoted by the vertical line marked $T_{0}$. Note the arrival of the surface ground motion at the antenna after $T_{0}$ at the $\mathrm{A20}, 19 \mathrm{X}$, and $18 \mathrm{R}$ sites. The signal quality at the CPP site is degraded by local wind noise. 


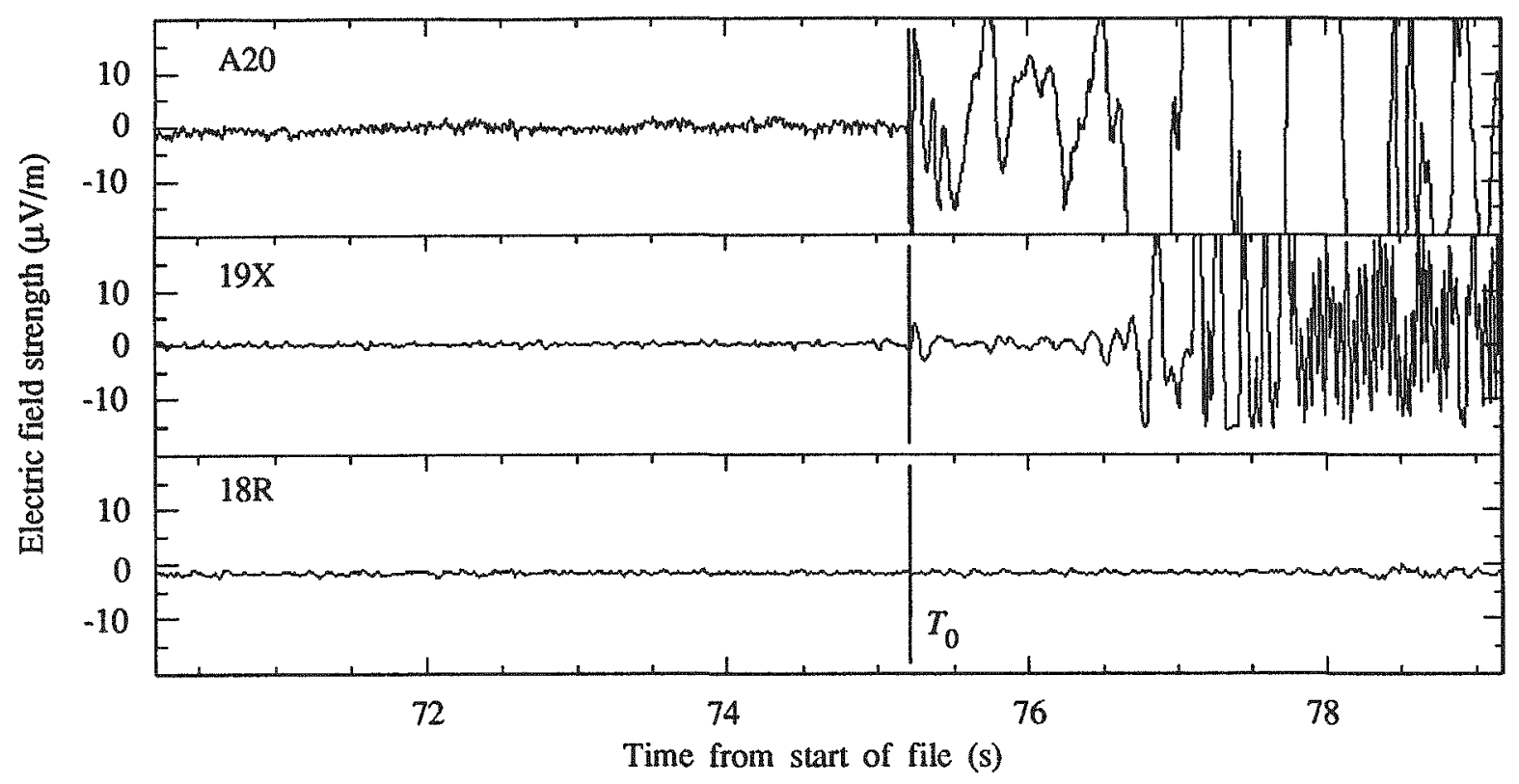

Figure 11. North electric $E_{\mathrm{n}}$ component of the ELF data from three sites, taken during the Hardin test. The detonation time is denoted by the vertical line marked $T_{0}$. Note the arrival of the surface ground motion at the $19 \mathrm{X}$ site after $T_{0}$.

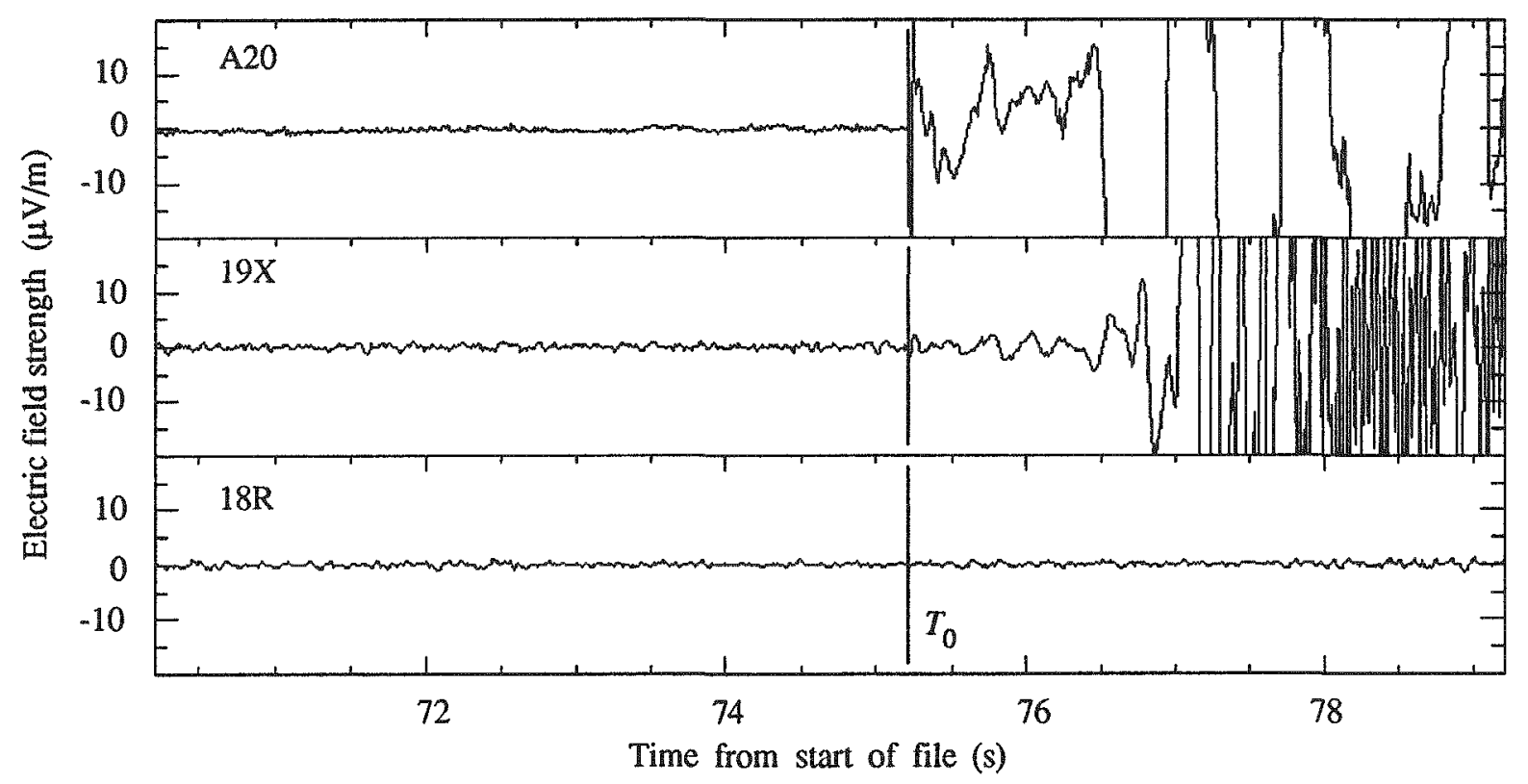

Figure 12. East electric $E_{\mathrm{c}}$ component of the ELF data from three sites, taken during the Har. din test. The detonation time is denoted by the vertical line marked $T_{0}$. Note the arrival of the surface ground motion at the $19 \mathrm{X}$ and $\mathrm{A} 20$ sites after $T_{0}$. 


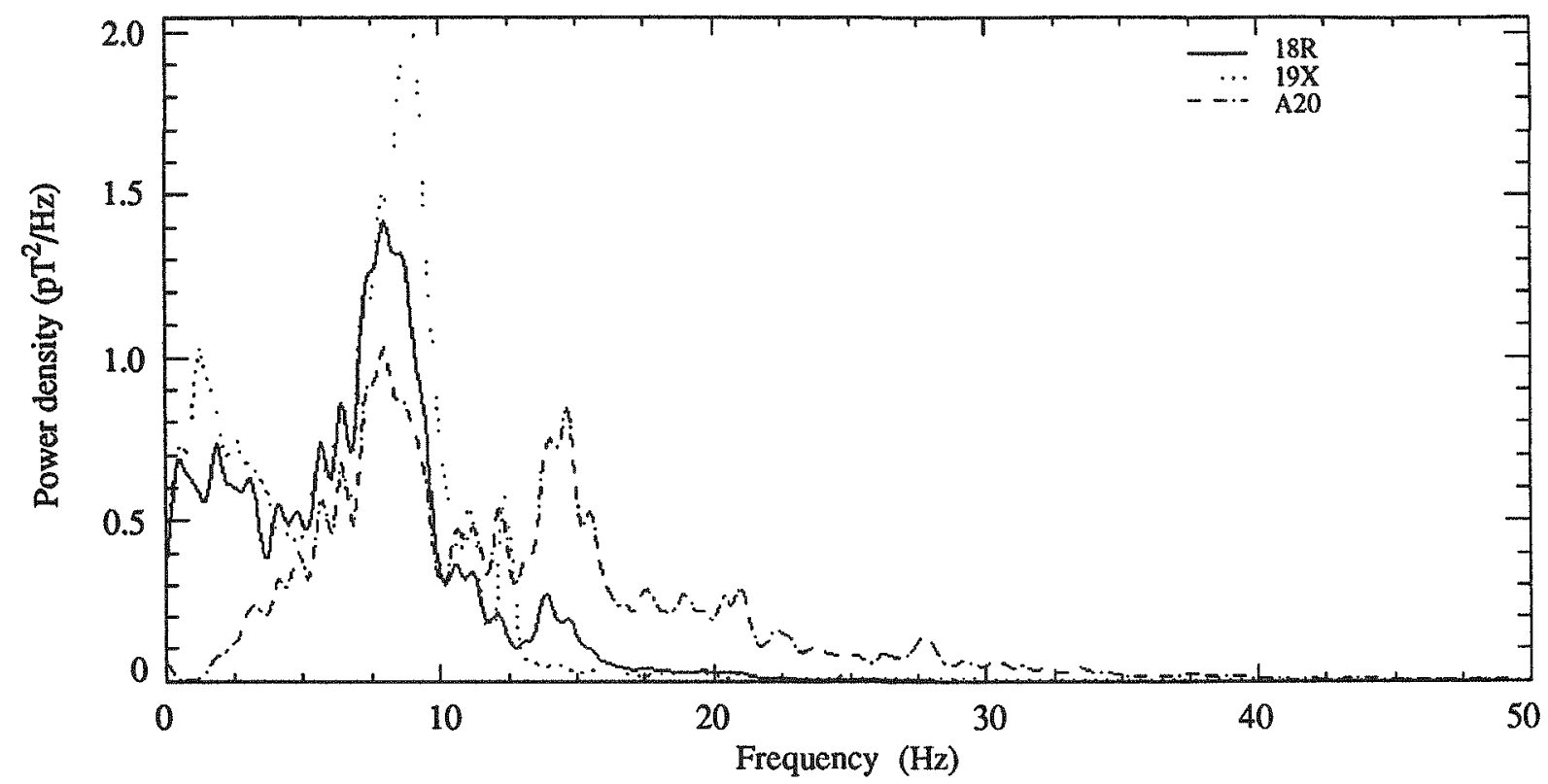

Figure 13. Comparison of power spectral densities for the north magnetic $B_{\mathrm{n}}$ component of the ELF data from the $18 R, 19 X$, and A20 sites before the Hardin test. PDR-2 data-acquisition equipment sampling at $100 \mathrm{~Hz}$ was used at $18 \mathrm{R}$ and $19 \mathrm{X}$. The IBM AT data-acquisition system was used at $\mathbf{A} 20$.

three Pahute Mesa stations at times of 600 to $800 \mathrm{~s}$ after $T_{0}$. These signals were especially prominent in the $B_{\mathrm{Z}}$ component, with amplitudes of $\sim 30$ to $40 \mathrm{pT}$ or more. My initial thought was that these signals could be related to the atmospheric acoustic pulse that travels vertically from surface ground zero and is generated by ground motion. The acoustic pulse strengthens and shortens as it travels upward through the decreasing gas density of the troposphere. The pulse arrives at and perturbs the ionosphere at times on the order of 600 to $800 \mathrm{~s}$ after $T_{0}$. This perturbation of the ionosphere, which has been extensively studied with ionosonds and radar, could conceivably create an ELF signal. I discussed these ideas with S. I. Warshaw of LLNL, who has created a computer code to model this ionospheric disturbance, and showed him the data. It was his opinion that the nature and timing of the signal were not consistent with a signal resulting from an ionospheric disturbance. We later confirmed this during the Kernville event; for this test, we co-located a seismometer with an ELF station $10 \mathrm{~km}$ from ground zero. We found that similar late-time magnetic-field ELF signals correlated exactly in time with late-time seismic events; the ELF signal was caused by local ground motion resulting from underground cavity collapse or local stress-relief microseisms.

\section{The Kernville Test}

The Kernville test was monitored by six ELF stations, the most stations to successfully operate in the series (see Table 3). The ground zero for the test was in the northwest part of NTS on Pahute Mesa. One station, HR, was $8.8 \mathrm{~km}$ south of ground zero. The other stations and their distance from ground zero were $18 \mathrm{R}$ ( $20 \mathrm{~km}$ to the south), A8 $(37.2 \mathrm{~km}$ to the east), FF $(86.6 \mathrm{~km}$ to the southeast), and the TM and CPP stations ( 420 and $485 \mathrm{~km}$, respectively). Stations $18 \mathrm{R}, \mathrm{TM}$, and CPP were three component stations $\left(E_{\mathrm{z}}, B_{\mathrm{n}}\right.$, and $\left.B_{\mathrm{e}}\right)$; the others measured six components, although the horizontal electric components at $A 8$ and the $B_{\mathrm{e}}$ component at $18 \mathrm{R}$ were inoperative because a coyote chewed on the signal cables during the night after the stations were set up. Two PDR-2 data recorders, each recording three channels and sampling at 200 $\mathrm{Hz}$, were used at the HR station. One PDR-2, recording three channels and sampling at $200 \mathrm{~Hz}$, was used at the $18 \mathrm{R}$ station. The $\mathrm{A} 8, \mathrm{FF}$, and TM stations were all operated by the UCLA team, using the IBM AT data-recording system.

The north magnetic component of the data from all six stations, starting $5 \mathrm{~s}$ before and ending $10 \mathrm{~s}$ after $T_{0}$, are shown in Fig. 15. There is a very clear signal at the closest station, HR, with about a 

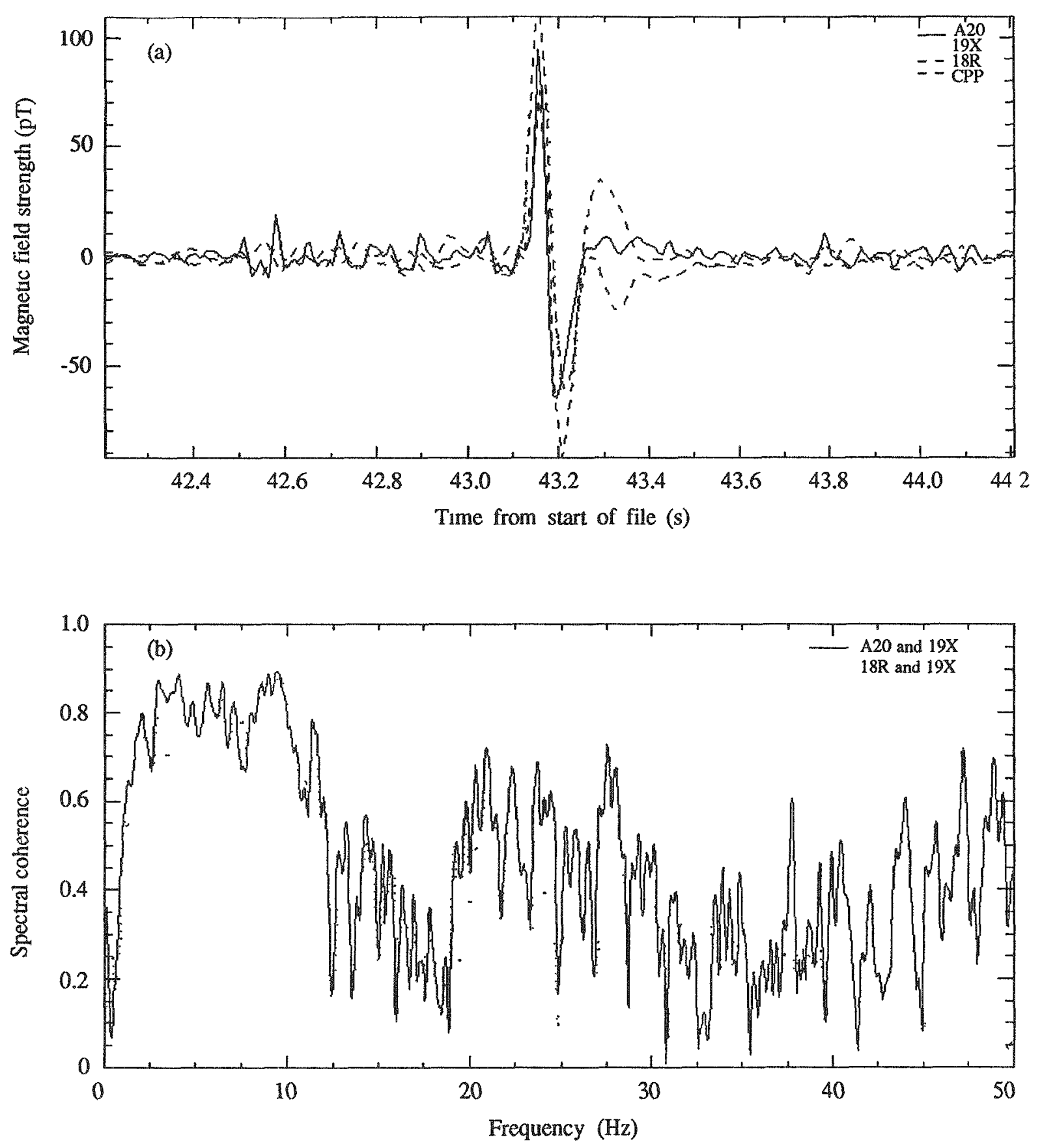

Figure 14. (a) The north magnetic ELF component of a large $Q$-burst recorded before the Hardin test at four different stations. This type of event can be used to test the accuracy of the relative time reference between stations. (b) Spectral coherence (a value of 1.0 represents identical signals) of the north magnetic ELF component between the A20 and 19X sites and the 18R and 19X sites on Pahute Mesa, recorded before the Hardin test. 


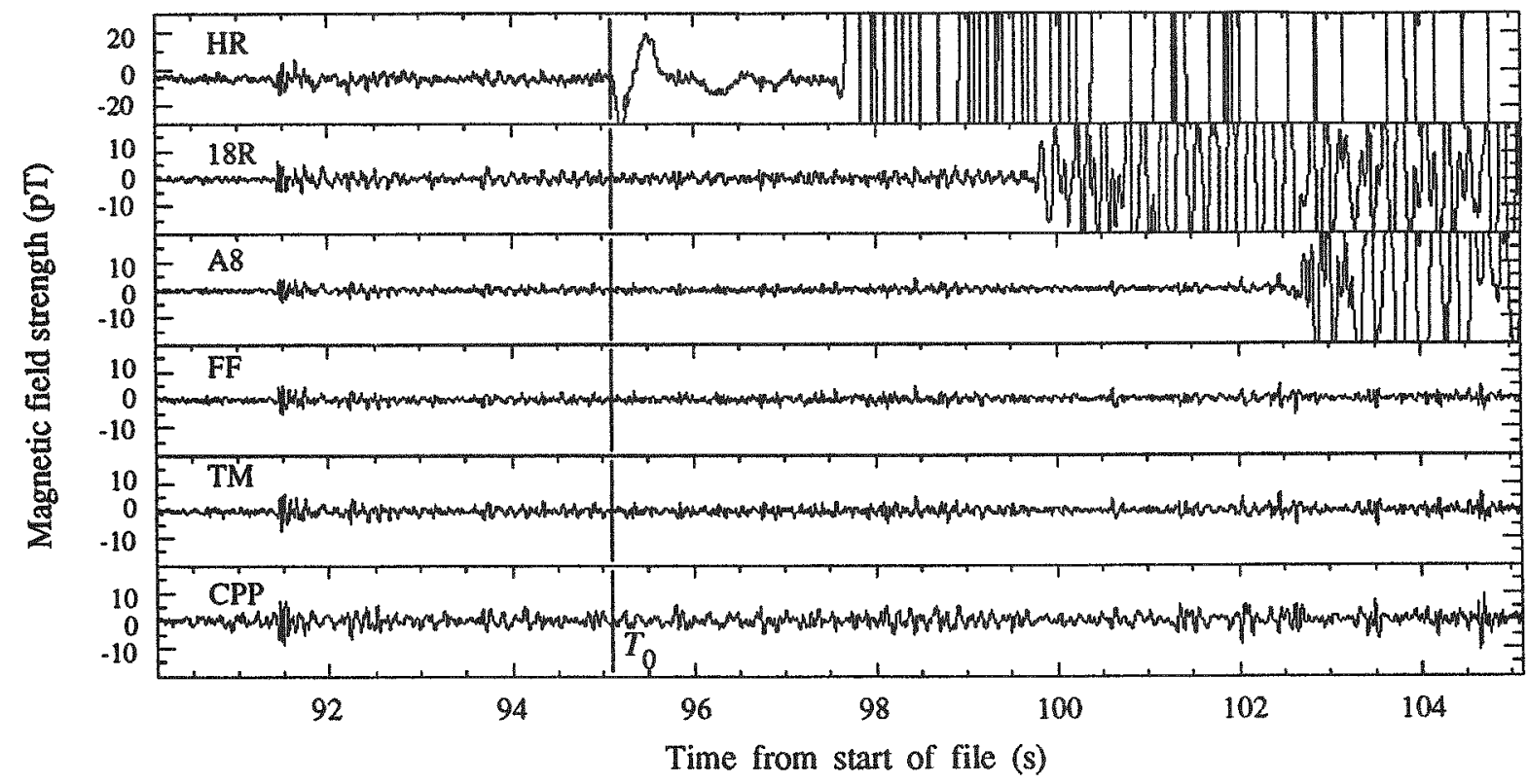

Figure 15. The north magnetic component $\mathrm{ELF}$ signals recorded from stations $\mathrm{HR}, 18 \mathrm{R}, \mathrm{A8}, \mathrm{FF}$, TM, and CPP during the Kernville test. Detonation time of the test is shown as $T_{0}$. The only site to show a signal at $T_{0}$ was the one closest to surface ground zero, $H R$ (at $8.8 \mathrm{~km}$ ). Note the wellcorrelated $Q$-burst occurring about $3.5 \mathrm{~s}$ before $T_{0}$ that was seen at all six sites.

10:1 signal-to-noise ratio. No similar signals are seen at $T_{0}$ on any of the other channels. The quality of the data is very good; a small $Q$-burst occurring about $3.5 \mathrm{~s}$ before $T_{0}$ is well-resolved in all of the channels. The surface ground motion affects the magnetometer at the following detonation times.

$$
\begin{aligned}
& T_{0}+3 \mathrm{~s} \text { at } \mathrm{HR} \\
& T_{0}+5 \mathrm{~s} \text { at } 18 \mathrm{R} \\
& T_{0}+7.5 \mathrm{~s} \text { at } \mathrm{A} 8
\end{aligned}
$$

The ground motion was measured at $T_{0}+13 \mathrm{~s}$ at the FF station but is not shown in Fig. 15.

Data from the six channels recorded at the HR station are shown in Fig. 16. The three magnetic channels (vertical $B_{\mathrm{z}}$, north $B_{\mathrm{n}}$, and east $B_{\mathrm{e}}$ ) all show a large signal at $T_{0}$, followed by the ground motion signal $3 \mathrm{~s}$ later. This ground motion signal is not very strong at the three electric field channels, which leads me to suspect the validity of these data. Problems with grounding have sometimes occurred with the electrical signals. These signals are very sensitive to current ground loops because of the high amplifier input impedances and high gains used. These current loops may have affected these data. Another possibility is that the PDR-2 data recorder may not have been operating properly. In any case, the electric data from this station are suspect.

Excellent data were obtained from the FF station (see Fig. 17). Several $Q$-bursts, from distant lightning discharges that correlate in the signals on all six channels, are shown. The most prominent bursts are those at

$$
T_{0}-3.5 \mathrm{~s}
$$

and

$$
T_{0}+11 \mathrm{~s}
$$

No signal was measured at $T_{0}$ on any of the channels. The ground motion affects the sensors $13 \mathrm{~s}$ after $T_{0}$; it is most prominent on the $B_{\mathrm{Z}}$ component and is only barely seen on the electric sensors at this distance $(86.6 \mathrm{~km})$. The data from the A8 station are quite similar to those from the FF station; no signal is present on any of the field components at $T_{0}$ at the $A 8, T M$, and CPP stations. The high correlation of the $B_{\mathrm{n}}$ background signals between the stations is shown in Fig. 18. The power density spectral coherence between the A8 and TM stations, the FF and TM stations, and the HR and 


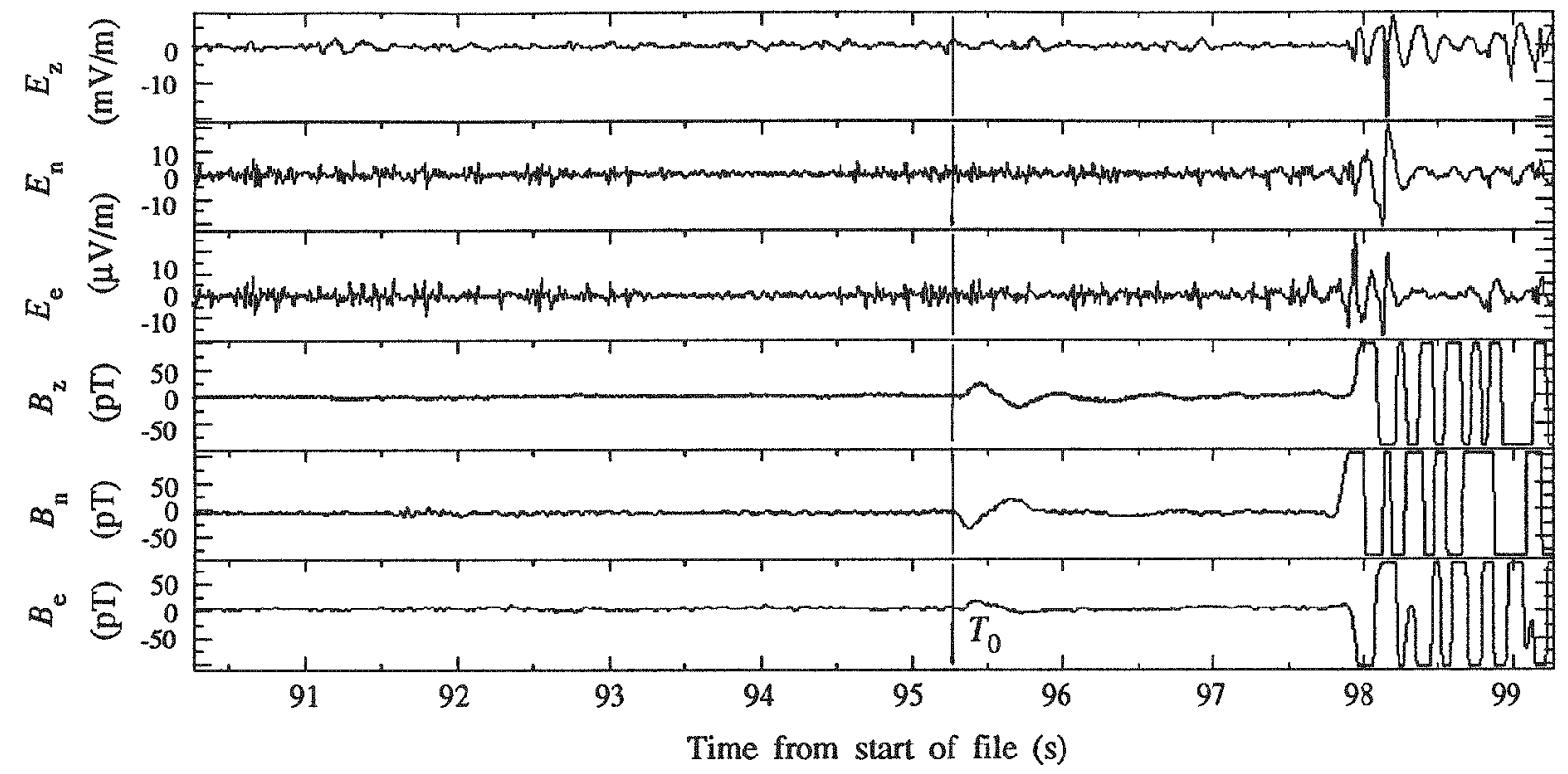

Figure 16. Six components $\left(E_{\mathrm{z}}, E_{\mathrm{n}}, E_{\mathrm{e}}, B_{\mathrm{z}}, B_{\mathrm{n}}\right.$, and $\left.B_{\mathrm{e}}\right)$ of $\mathrm{ELF}$ data recorded at the $\mathrm{HR}$ site during the Kermville test. Detonation time is marked by $T_{0}$. The surface ground motion signal arrives about $2.5 \mathrm{~s}$ after $T_{0}$ and markedly perturbs the field sensors.

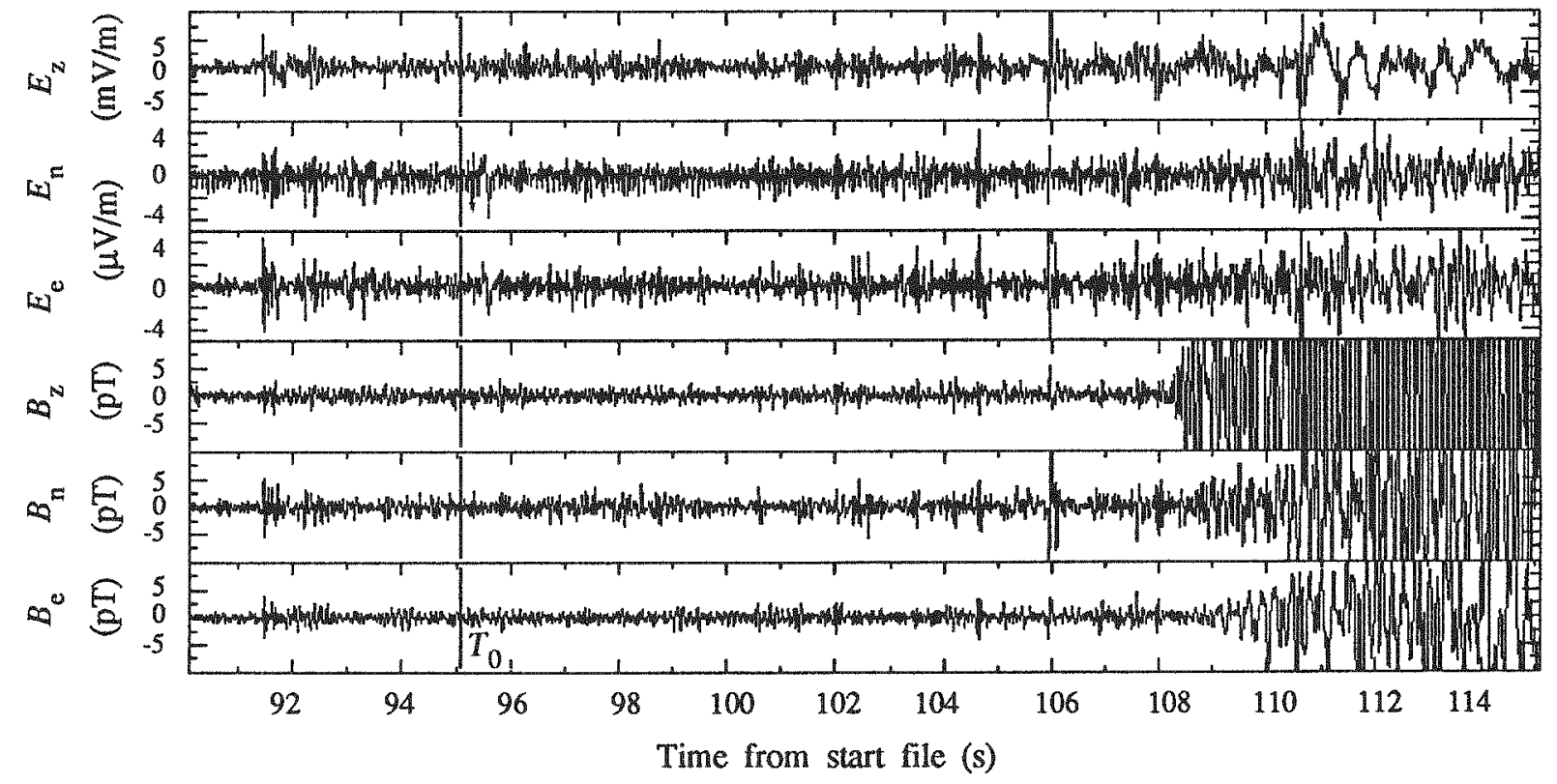

Figure 17. Six components $\left(E_{\mathrm{z}}, E_{\mathrm{n}}, E_{\mathrm{e}}, \boldsymbol{B}_{\mathrm{z}}, \boldsymbol{B}_{\mathrm{n}}\right.$, and $\left.\boldsymbol{B}_{\mathrm{e}}\right)$ of ELF data recorded at the FF site during the Kernville test. Detonation time is marked by $T_{0}$. The surface ground motion signal arrives about $13.2 \mathrm{~s}$ after $T_{0}$ and markedly perturbs the magnetic field sensors. At this distance $(86.6 \mathrm{~km})$, the ground motion does not perturb the electric field sensors as strongly. Note the $Q$-bursts recorded $3.5 \mathrm{~s}$ before and $11 \mathrm{~s}$ after $T_{0}$. 


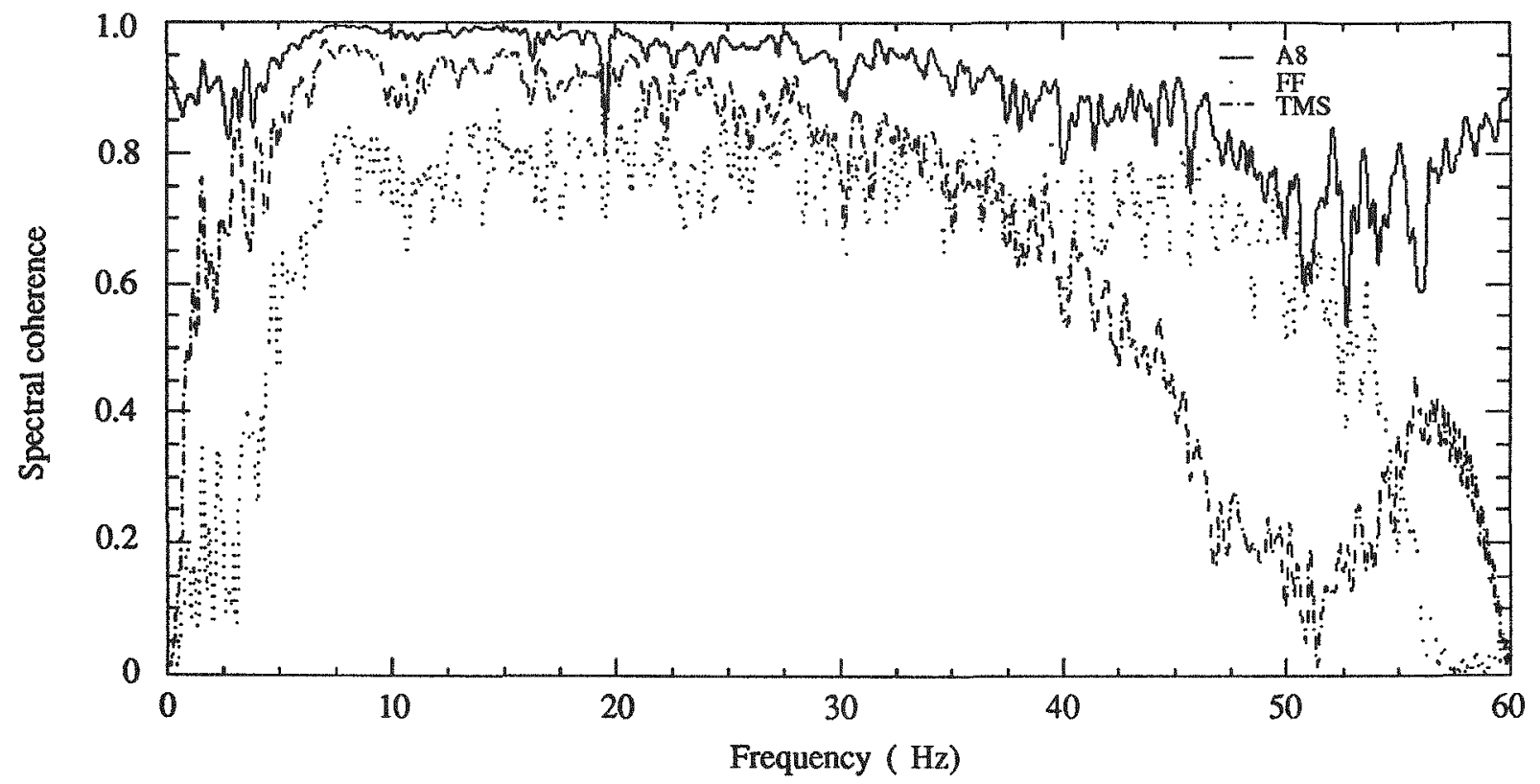

Figure 18. Comparison of the spectral coherence of the north magnetic $B_{\mathrm{n}}$ ELF component between different stations before the Kermville test. The solid curve compares the A8 and TMS stations, separated by $\sim 460 \mathrm{~km}$. The dotted curve compares the FF and TMS stations, separated by $\sim \mathbf{4 2 0} \mathrm{km}$. The dashed curve compares the HR and TMS stations, separated by $\sim 480 \mathrm{~km}$.

TM stations is 0.7 or greater (a value of 1.0 indicates identical signals at a particular frequency) between 7 and $40 \mathrm{~Hz}$. Bear in mind that the HR and TM stations have completely different datarecording, amplifying, and filtering equipment and are separated from each other by $>420 \mathrm{~km}$. The improvement in the bandpass capabilities of the PDR-2 equipment by sampling at $200 \mathrm{~Hz}$ is shown in Fig. 19. The $B_{\mathrm{n}}$ channel of the $18 \mathrm{R}$ and $\mathrm{HR}$ stations can now resolve the second $(13.5$ to $14 \mathrm{~Hz})$ Schumann resonance, and partially resolve the third $(20 \mathrm{~Hz})$ resonance. The spectral responses of the PDR-2 stations, HR and 18R, are now much more like those for IBM AT stations, such as A8 [see Fig. 19(c)].

The Kernville test was the first to confirm that ELF signals were not detected at distances $>10 \mathrm{~km}$ from ground zero; before this test, the data were somewhat confusing and ambiguous. Tests after Kernville (Shellbourne and Comstock) verified this conclusion. There remained one final question to be checked out. If the source of ELF energy from an underground nuclear device acts as either a magnetic or an electric dipole, then, for the fundamental mode, some of the field components will be null at the source and have a maximum strength $90^{\circ}$ of arc away from the source. Therefore, it is possible that no
ELF signals would be seen at distances $>10 \mathrm{~km}$ away from the near field, but still within a few hundred kilometers of the source, although they may be detected at greater distances. The JVE gave us the opportunity to monitor tests at greater distances. Scientists from the USSR monitored the Kearsarge test at NTS and scientists from the USA monitored the Shagan test at the Shagan River site in the Semipalatinsk region of the Soviet Union.

\section{The JVE Tests}

We decided to install a temporary ELF station near Fairbanks, Alaska to monitor ELF Schumann components $\left(E_{\mathrm{z}}, B_{\mathrm{n}}\right.$, and $\left.B_{\mathrm{e}}\right)$ for both of the JVE underground nuclear tests. NTS is $\sim 90^{\circ}$ of arc $(10,000$ $\mathrm{km}$ ) from Semipalatinsk. Fairbanks is $6600 \mathrm{~km}$ from Semipalatinsk and $3800 \mathrm{~km}$ from NTS. Thus, Fairbanks is at an intermediate distance for tests at both NTS and Semipalatinsk. Because of terrain limitations and because we were primarily interested in monitoring the fundamental modes (Schumann components) of the earth-ionosphere cavity, we designed the FB station to only monitor three components. As on previous tests, two NTS stations (FF and A8) were staffed by the UCLA team. The CPP ELF site at LLNL was also in operation for the JVE 


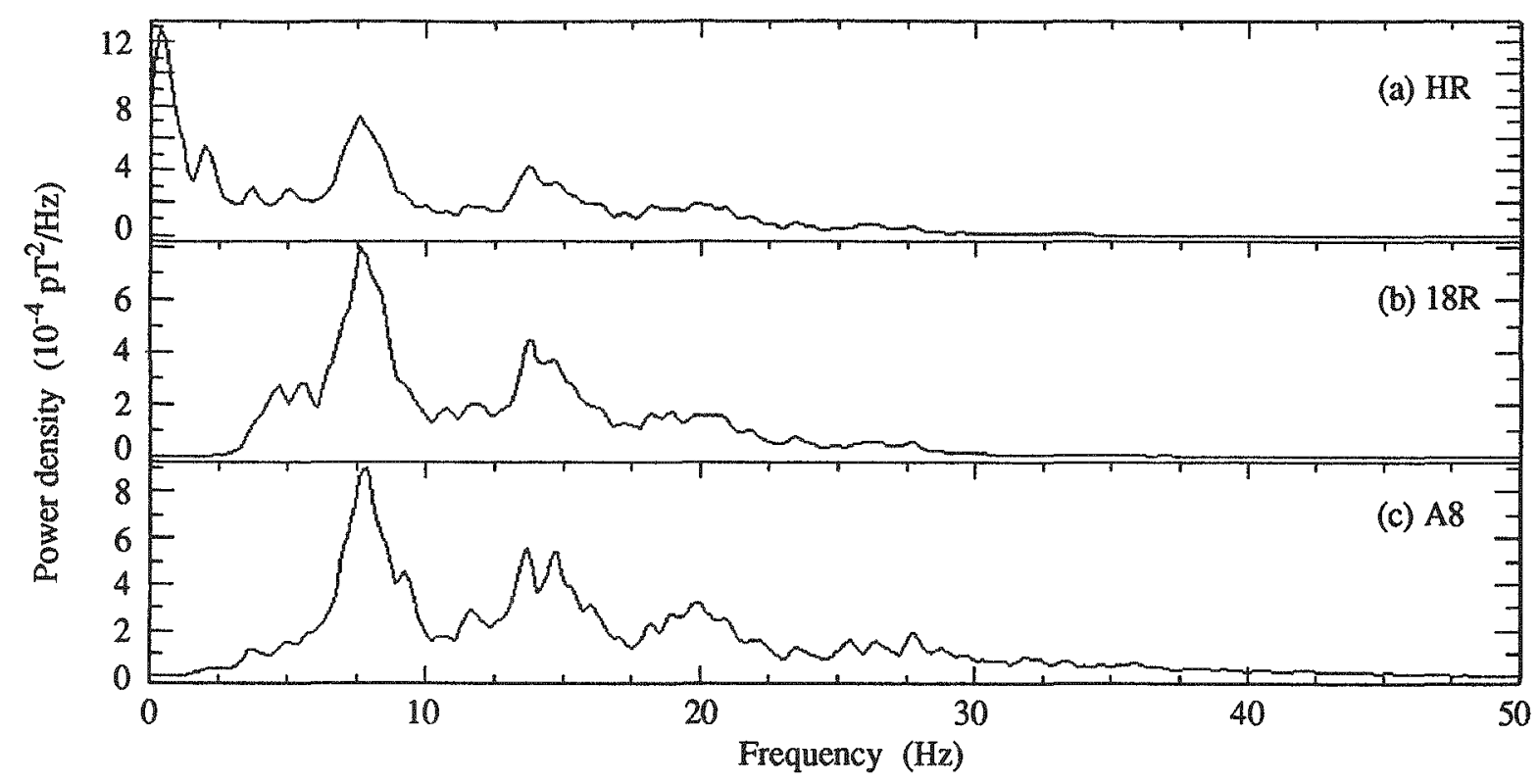

Figure 19. Power spectral densities for the north magnetic $B_{\mathfrak{n}}$ ELF component recorded at three sites before the Kernville test. PDR-2 data recorders sampling at $200 \mathrm{~Hz}$ were used at the $18 \mathrm{R}$ and HR sites. The IBM AT data-recording system was used at the A8 site.

tests, although local interference made these data unusable for the Shagan test. The site at A8 was inoperative for the Kearsarge test. The sites at NTS and FB had the IBM AT data-acquisition system and the site at CPP had a PDR-2.

The north magnetic $B_{\mathrm{n}}$ component data from the three stations operating during the Kearsarge test are shown in Fig. 20. Note the high correlation between stations separated by as much as $3800 \mathrm{~km}$. There are several $Q$-bursts visible in Fig. 20, most notably at 147,148 , and $149 \mathrm{~s}$ after the start of the file. The largest $Q$-burst, which looks like a typical double-pulse resulting from a large lightning primary and return stroke, occurs just before $T_{0}$. A signal related to the Kearsarge test is probably not present in Fig. 20. The three Schumann components $\left(E_{\mathrm{z}}, B_{\mathrm{n}}\right.$, $B_{\mathrm{e}}$ ) are shown for both the FB and FF stations in Fig. 21. The correlation of $Q$-bursts in all three field components are clearly visible. There is no signal in Fig. 21 that can be clearly identified as being related to the Kearsarge test. It is also evident from Fig. 21 that both the FB and FF stations collected data of excellent quality.

The three-component station at Fairbanks, FB, and the 2 six-component stations at NTS were in operation for the Shagan test. The $B_{\mathrm{n}}$ component data for the three stations, for $5 \mathrm{~s}$ before and after $T_{0}$, are shown in Fig. 22. The three Schumann components $\left(E_{\mathrm{z}}, B_{\mathrm{n}}\right.$, and $\left.B_{\mathrm{e}}\right)$ are shown in Fig. 23 for the FF and FB stations, $5 \mathrm{~s}$ before and after $T_{0}$. Note again the high correlation of the north magnetic component of the signals. A comparison of the spectral coherence between FB and FF for the $B_{\mathrm{n}}$ and $B_{\mathrm{e}}$ components is shown in Fig. 24. In Figs. 22 and 23 , a large $Q$-burst occurs $\sim 1 \mathrm{~s}$ before $T_{0}$. The A8 and FF data show the $Q$-burst to have a sharp onset with ringing afterwards at 7 to $8 \mathrm{~Hz}$. The $Q$-burst is also seen in the FB data, but it does not have as sharp an onset; this may be because the different systems have different bandpasses. The strong $Q$-burst dies out by $T_{0}$. There is a pulse in the FB data about $100 \mathrm{~ms}$ after $T_{0}$ that is not seen in the data from FF or A8. It is possible that this pulse is related to the test, but it is more likely that it has a source local to the FB station, which would explain why it is not seen in any of the six components at the FF or $\mathrm{A} 8$ stations. The pulse detected $100 \mathrm{~ms}$ after $T_{0}$ at FB is not detected at FF. It is also notable that the same pulse has a much larger $B_{\mathrm{n}}$ component than $B_{\mathrm{e}}$ component. This would be consistent with the source being in the direction of Semipalatinsk; but, if the signal really represents an excitation of the earth-ionosphere cavity, some component of it should be seen at the NTS stations. The official time given for this Soviet event is 0400:00.00 UT on Sept. 14, 1988. 


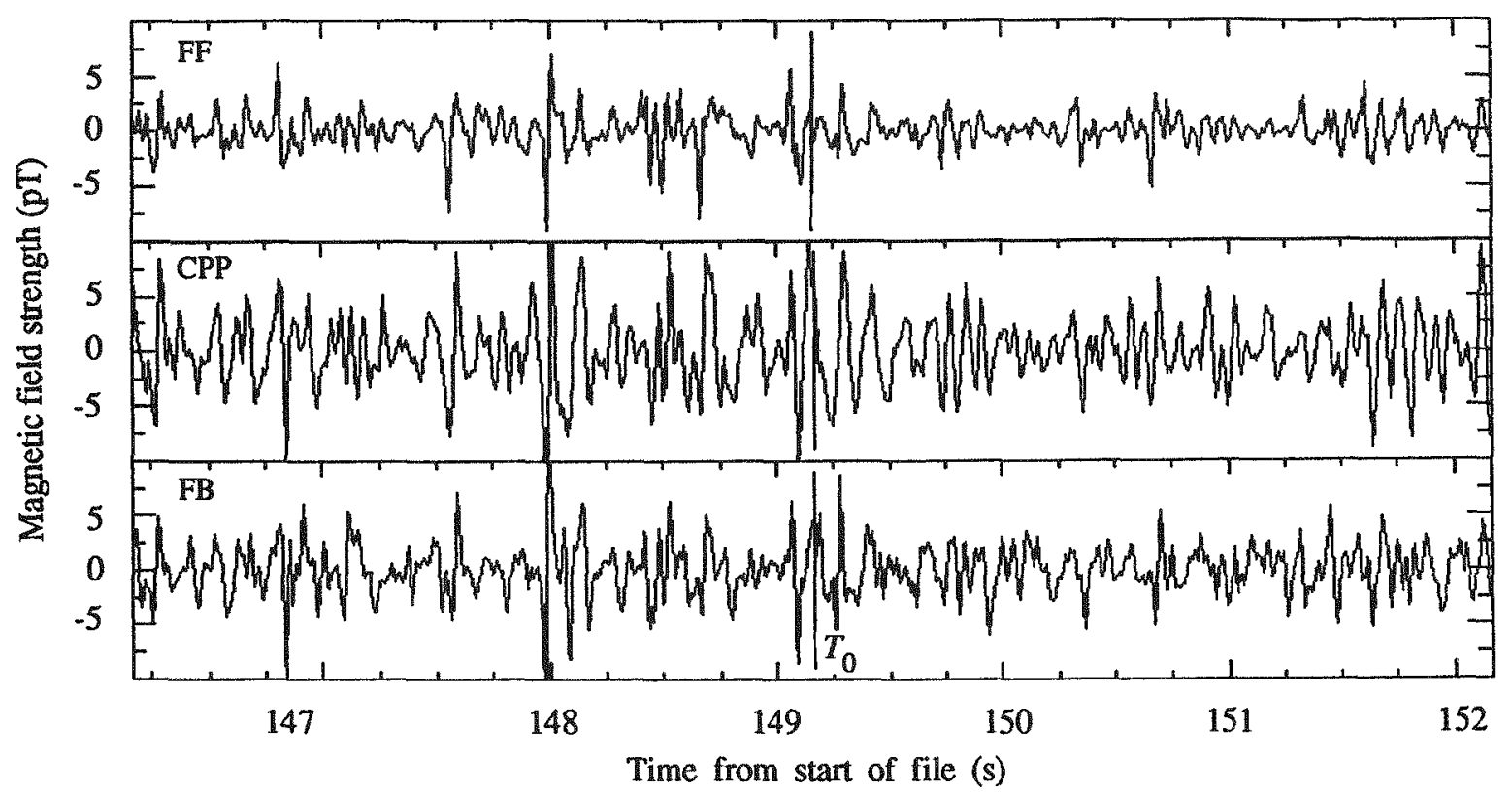

Figure 20. North magnetic $B_{\mathrm{n}}$ ELF component signals recorded at the FF, CPP, and FB sites during the Keasarge JVE test. Note the $Q$-bursts occurring at $146.75,148$, and $149 \mathrm{~s}$ into the record.

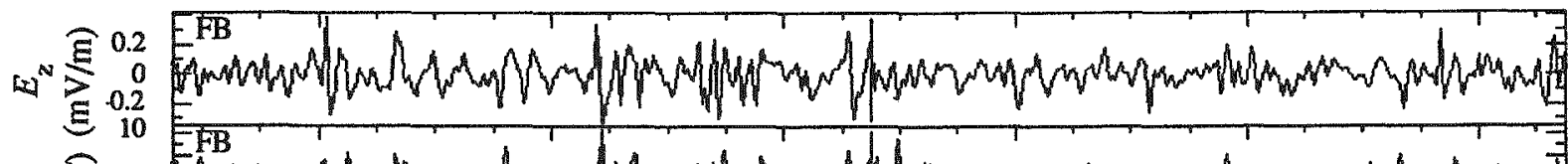
a. G.

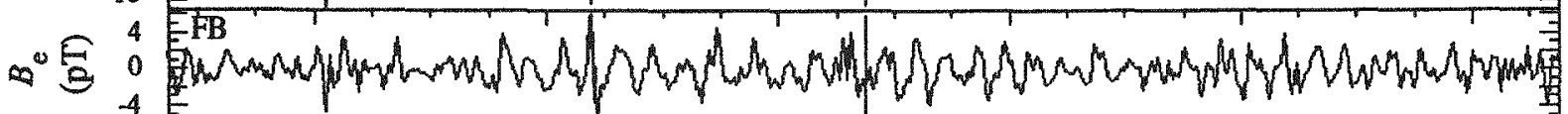
w" E⿱ Q.G.

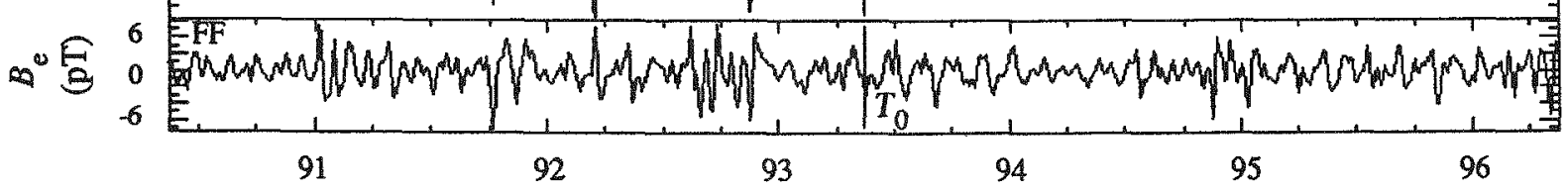
Time from start of file (s)

Figure 21. The three Schumann components $\left(E_{\mathrm{z},} B_{\mathrm{n}}\right.$, and $\left.B_{\mathrm{e}}\right)$ of the ELF data recorded at the FB and FF sites during the Kearsarge test. Note the $Q$-bursts that are also shown in $\mathbf{F i g} .20$. 


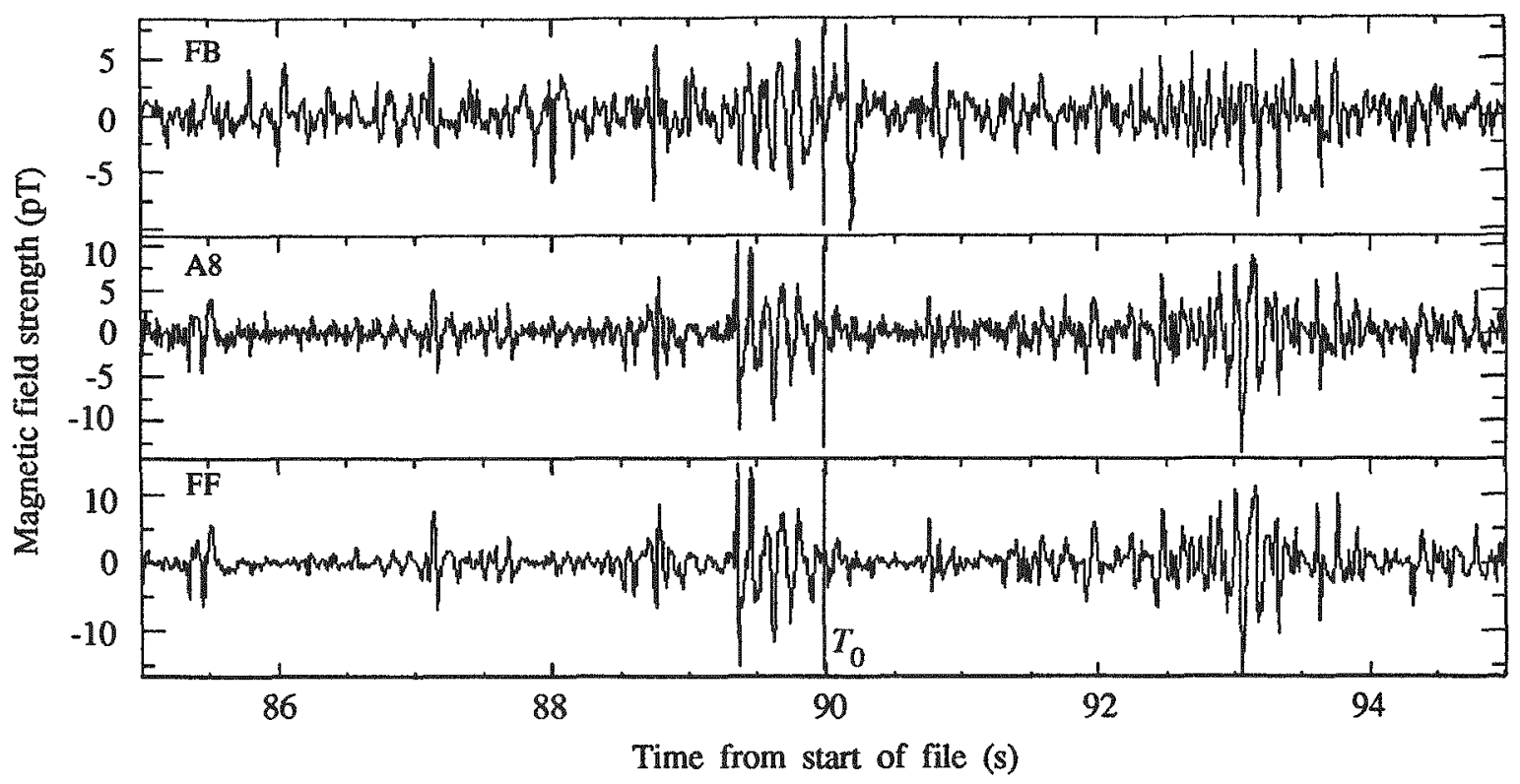

Figure 22. The north magnetic $B_{\mathrm{n}} \mathrm{ELF}$ data recorded at the $\mathrm{FB}, \mathbf{A 8}$, and $\mathrm{FF}$ sites during the Shagan test near Semipalatinsk. Note the $Q$-bursts occurring at $87,88.4,88.8,90.7$, and $93 \mathrm{~s}$ into the file. The $Q$-burst occurring just before $T_{0}$ is especially strong.

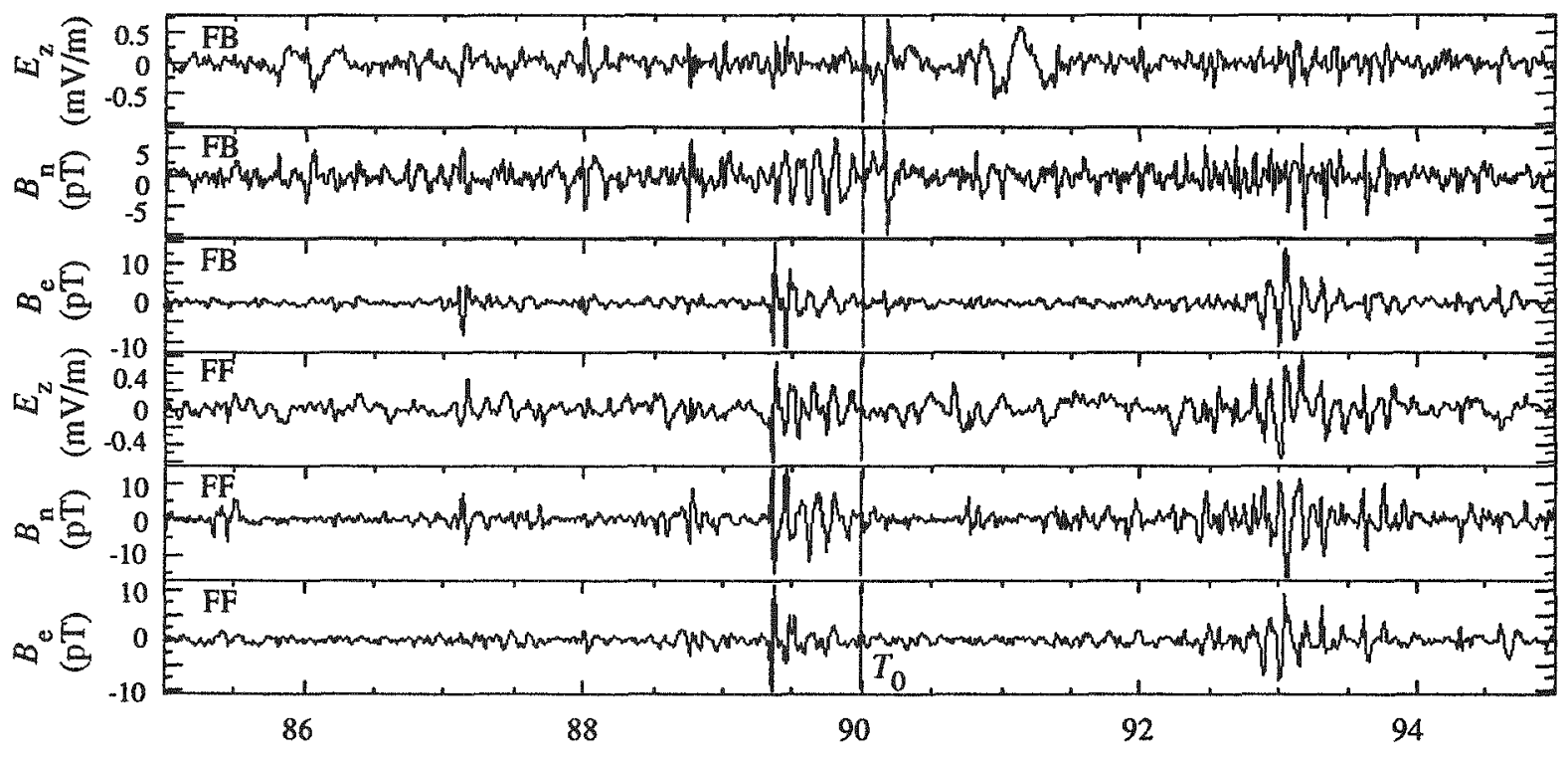

Time from start of file (s)

Figure 23. The three Schumann components $\left(\boldsymbol{E}_{z}, \boldsymbol{B}_{\mathrm{n}}\right.$, and $\left.\boldsymbol{B}_{\mathrm{e}}\right)$ of the ELF data recorded at the FB and FF sites during the Shagan test near Semipalatinsk. Note the $Q$-bursts that are also shown in Fig. 20. Detonation time is denoted by $T_{0}$. 


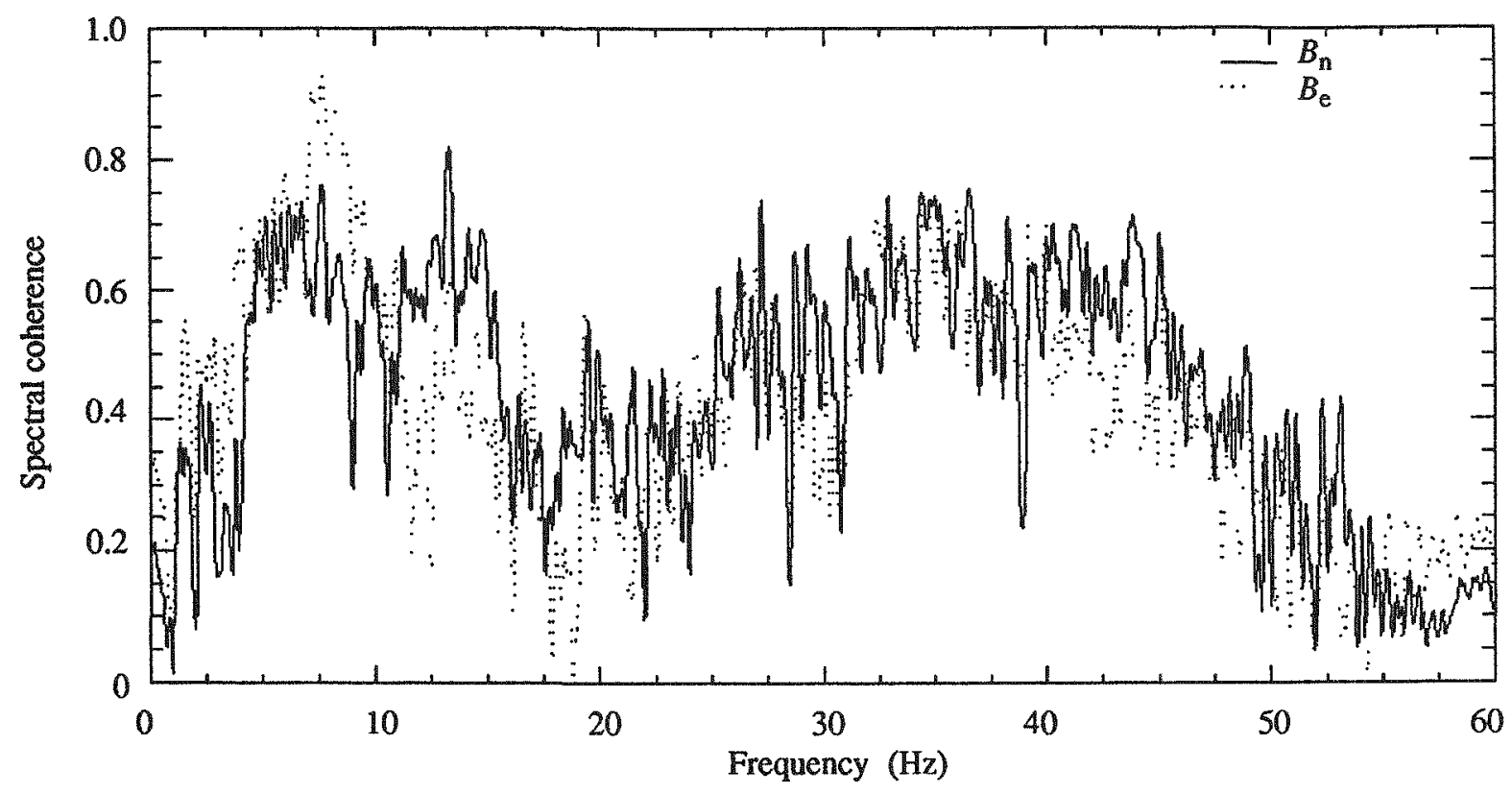

Figure 24. Comparison of spectral coherence of the $B_{\mathrm{n}}$ and $B_{\mathrm{e}} \mathrm{ELF}$ components recorded before the JVE test between the FB and FF sites, which are separated by $3800 \mathrm{~km}$.

However, we do not know how the Soviets determine the timing for their tests. There may be systematic differences between their reference time and ours, so the fact that the pulse in question occurs $100 \mathrm{~ms}$ after the official $T_{0}$ may not be significant. Thus, we cannot know if the pulse 100 $\mathrm{ms}$ after $T_{0}$ is from the Shagan test. If the pulse is from the test, the signal amplitude is still well within the level of the background noise.

\section{Additional Comments}

Table 3 shows that there were only two tests, Belmont and Hardin, for which near-field signals were detected at more than one site. During Belmont, three sites detected signals, but the A18 site only detected signals in the $B_{\mathrm{n}}$ and $B_{\mathrm{z}}$ components. The electric signals for Belmont at the EG and $18 \mathrm{R}$ sites are probably not reliable. Therefore, the only really good six-component data for this test comes from the $A 20$ site.

Another factor to consider in analyzing these data is that the A20 and EG sites used different detection systems (e.g., the bandpass characteristics are different); thus, it is difficult to compare the results from each site. During the Hardin test, nearfield data were collected at A20 and 19X; each site had different detection systems. I used the field calculations made by Burke and shown in Appendix A in an attempt to draw some conclusions from our data about the nature of the source for these tests. However, we do not have sufficient data to make any conclusion other than to say that these results may be most consistent with the source being a horizontal magnetic dipole since all six components of the field were detected for each test at A20. With only one or two reliable measurement sites in the near field for each test, it is impossible to determine if there is any azimuthal dependence of the fields. Theory (see Ref. 11 and Appendix A) suggests that field strength decreases as the negative two or three power of distance from the source. Our data seem to be consistent with theory, but there were not enough stations of a similar type deployed at the same azimuth and at different distances to truly test this theory.

I also attempted to analyze the data in a way that would enhance the signal-to-noise ratio of the detectors. By averaging data from one component, for instance $B_{\mathrm{n}}$, at two or more stations where the same type of detection system was used, it is theoretically possible to decrease the incoherent background noise in the detectors. In practice, I found that differences in site-specific conditions, such as local wind or the proximity to local thunderstorms, were the controlling factors governing the site's noise level. These effects could only be minimized by co-locating several stations and then averaging the results. Such procedures are beyond the scope of this investigation. 
Sentman took the data from this study and carried out a theoretical analysis to determine the nature of the strength of the background ELF signal caused by worldwide lightning activity. His report is Appendix B. Sentman notes that the ELF signals that we have detected from underground nuclear explosions are of longer duration, by a factor of $\sim 500$, than ELF signals from individual lightning discharges. Thus, ELF signals from nuclear explosions have a much lower frequency content than do lightning discharges and are much less efficient in exciting the earth-ionosphere resonant cavity. Based on our experimental results, he compared the field strength for a hypothetical vertical electric current dipole from an underground nuclear test $\left(5 \times 10^{8} \mathrm{~A} \cdot \mathrm{m}\right)$ with a typical lightning current moment $\left(6 \times 10^{8}\right.$ Amm). Because of the lower efficiency of excitation, the distant ELF field strength resulting from the underground test is $>100$ times weaker than the background field emanating from lightning activity. This probably explains why we have been unable to detect ELF signals from underground tests when the detection equipment is located far from the near field.

\section{Conclusions}

Over the three years of this study, we have greatly improved our techniques for measuring and interpreting EM signals in the lower end of the ELF frequency range. With our present capabilities, we are confident that we can detect ELF signals in the near field as well as those that excite the earthionosphere resonant cavity. From the results of this investigation, we conclude that underground nuclear tests of the type we have monitored for this project do not create an EM signal sufficient to excite the
Schumann resonances. Thus, ELF monitoring at frequencies below $60 \mathrm{~Hz}$ has not been demonstrated to be useful for regional or global-scale monitoring of underground nuclear tests. There is a possibility that careful measurements in the near field $(<10 \mathrm{~km}$ from ground zero) could lead to using ELF measurements to estimate yields of underground tests, but much more research will have to be carried out to better understand the cause of the signals and their near-field behavior.

\section{Acknowledgments}

Motivation for this project originated at LLNL with the thoughts and enthusiasm of David S. Dearborn, who was also involved in some of the original field testing of the ELF sensing equipment. Most of the difficult early work in this project was done by P. W. Rodgers also from LLNL; I primarily followed in his footsteps in completing this project over the last two years.

Most of the LLNL technical work, including design and fielding of the pre-amplifiers and filters used in the LLNL data-collection system, was carried out most capably by Larry Wethern. Don Rock and Dan Ewert helped fill in during deployments at NTS when Larry was unable to be there. Don Jackson, of EG\&G at NTS, was exceedingly helpful in providing support for the remote triggering of our equipment at NTS and in generally facilitating our work at NTS. Additional support at NTS was provided by the LLNL operations staff, most notably Leo McGurk.

The cooperation, enthusiasm, and support of D. D. Sentman and F. R. George from UCLA, went far beyond mere contractual obligations. They provided a significant portion of the data obtained by this study as well as invaluable technical expertise. Guidance and encouragement throughout this project was provided by Robert Geil and Milo Nordyke of LLNL. Funding was provided by the Department of Energy through the Office of Arms Control. 


\section{References}

1. D. D. Sentman, "Schumann Resonance Effects of Electrical Conductivity Perturbations in an Exponential Atmospheric/lonospheric Profile," J. Atmos. Terr. Phys. 45 (1), 55-65 (1983).

2. D. D. Sentman, "Magnetic Elliptical Polarization of Schumann Resonances," Radio Sci. 2 (4), 595-606 (1987).

3. D. D. Sentman, "New Technique for Detecting Line Splitting Using Discrete Schumann Resonance Excitations," submitted to Geophys. Res. Lett. (1988).

4. D. D. Sentman, "Polarity of Ultralarge Lightning Strokes Inferred from Discrete Schumann Resonance Transients," (Abstract) EOS Trans. of the Amer. Geophys. Union 69 (44), 1050 (1988).

5. W. O. Schumann, "Uber die strahlungslosen Eigenschwingungen einer leitenden Kugel, die von einer Luftschicht un einer Ionospharenhulle umgeben ist," Z. Naturforsch. 7a, 149 (1952).

6. M. Balser and C. A. Wagner, "Observations of Earth-Ionosphere Cavity Resonances," Nature (London) $188,368(1960)$.

7. C. Polk, "Schumann Resonances," CRC Handbook of Atmospherics, H. Volland, Ed. (CRC Press Inc., Boca Raton, Florida, 1982), pp. 111-178.

8. D. D. Sentman, Institute of Geophysics and Planetary Physics, University of California Los Angeles, personal communication (1988).

9. T. Ogawa, "The Lightning Current," CRC Handbook of Atmospherics, H. Volland, Ed. ( CRC Press Inc., Boca Raton, Florida, 1982), pp. $23-64$.

10. M. Balser and C. A. Wagner, "Effect of a High-Altitude Nuclear Detonation on the Earth-Ionosphere Cavity," J. Geophys. Res. 68 (13), 4115-4118 (1963).

11. C. J. Zablocki, "Electrical Transients Observed during Underground Nuclear Explosions," J. Geophys. Res. 71 (12), 3523-3542 (1966).

12. J. Malik, R. Fitzhugh, and F. Hormuth, Electromagnetic Signals from Underground Nuclear Explosions, Los Alamos National Laboratory, Los Alamos, New Mexico, LA-10545-MS (1985).

13. S. T. Martner and N. R. Sparks, "The Electroseismic Effect," Geophysics 24 (2), 297-308 (1959).

14. D. D. Sentman and F. R. George, "A Microcomputer-Controlled, Real-Time Data Acquisition and Analysis System for Schumann Resonance Measurements," submitted to IEEE Transactions on Geoscience and Remote Sensing (1988).

15. D. D. Sentman, "PC Monitors Lightning Worldwide," Computers in Science 1 (1), 25 (1987).

16. H. F. Morrison, U. Conti, V. F. Labson, E. Nichols, and N. E. Goldstein, Field Tests of Noise in Squid and Induction Coil Magnetometers, Lawrence Berkeley Laboratory, Berkeley, Calif., LBID-901 (1984). 


\title{
Appendix A
}

\section{Source Modeling of Event Induced ELF Radiation}

\author{
Jerry Burke
}

Engineering Research Division Electrical Engineering Department, Lawrence Livermore National Laboratory Livermore, California 94551 


\section{SOURCE MODELING OF EVENT INDUCED ELF RADIATION}

\section{Introduction}

Extra low frequency (ELF) radiation has been measured from a number of nuclear events and is under investigation as a means of detecting such events. The source of this radiation has not yet been determined, but candidate mechanisms include the magnetic dipole due to exclusion of the Earth's magnetic field, asymmetrical electron flux, and currents in the up-hole emplacement pipe. Each of these mechanisms involves magnetic or electric sources radiating near the Earth's surface. Hence, as an initial attempt to understand this radiation we have evaluated the fields from point sources radiating near the air-ground interface. At the ELF frequencies of interest these fields can be evaluated accurately from relatively simple quasistatic approximations.

A detailed analysis of the phenomenon including all of the physics involved could be very difficult. However, in many cases the magnitude of possible sources can be estimated. For example, if the current is known at a point on an emplacement pipe the current distribution can be approximated as a piecewise sinusoidal function with the known propagation constant for the ground or air. Furthermore, the distribution of source currents in the earth, within a few hundred meters of the surface, is electrically small at the ELF frequencies of interest. Hence the sources can reasonably be lumped as point dipoles, and the primary questions to be answered are the dipole moment, time response, orientation and type, electric or magnetic, of the sources.

As a first step in determining the possible source mechanism for ELF radiation we have generated plots of the components of electric and magnetic field due to electric and magnetic dipoles in the ground. These fields are plotted as a function of distance and frequency and are used to determine the transient fields due to a step function source. A similar study of the elementary dipole fields was done by Zablocki [1] in 1966 in an attempt to understand event induced ELF measured at that time. Several field components in that study appear to be inaccurate or reversed in sign. However, these errors do not appear to change the conclusions of that study, which were inconclusive.

\section{The Field of a Source Near an Interface}

The solution for the field of a point source near an interface was developed by Arnold Sommerfeld in 1909. Sommerfeld expanded the fields in cylindrical-wave functions and matched boundary conditions at the interface to obtain solutions in the form of inverse Hankel transforms, now often called Sommerfeld integrals. While these integrals cannot be evaluated in closed form, approximations have been developed for various conditions such as high contrast between the media and large or small separation of the source and evaluation points.

Numerical evaluation of the integrals can provide accurate results over a wide range of parameters, although with more complexity and computation time than for typical approximations. Codes for numerical evaluation of the Sommerfeld integrals have been 
developed at LLNL for use in method-ofmoments codes for antenna modeling. The most general such code, described in [2], can evaluate the field of electric or magnetic dipoles in a horizontally stratified medium for arbitrary position of source and evaluation points. These codes integrate in the complex plane along contours that approximate steepest descent paths from the saddle points. Since they do not follow the exact steepest descent path the separation of source and evaluation points is limited to about twenty wavelengths before computation time becomes excessive. The codes are accurate for the conditions occurring in this ELF source study, including frequencies down to one Hertz.

Relatively simple approximations valid at ELF are provided by the quasistatic formulas developed by J.R. Wait and others as cited by Zablocki [1]. A summary of the quasistatic formulas given by Kraichman as Table 3.8 in [3] is more accurate and complete than the equations used by Zablocki. These are repeated here since they were used for most of the plots that follow. Kraichman has the horizontal magnetic dipole oriented along the $y$ axis and the horizontal electric dipole along the $x$ axis. The equations have been modified here to put the horizontal magnetic dipole along the $x$ axis as shown in Fig. 1. The fields are in cylindrical components $\rho, \phi$ and $z$ indicated by subscripts, and the source is indicated by superscripts $V$ or $H$ for vertical or horizontal orientation and $E$ or $M$ for electric or magnetic dipole. The propagation constant in air is $\gamma_{0}=j \omega\left(\mu_{0} \epsilon_{0}\right)^{1 / 2}$ and that in the ground is $\gamma_{1}=\left(-\omega^{2} \mu_{1} \epsilon_{1}+j \omega \mu_{1} \sigma_{1}\right)^{1 / 2}$ where $\epsilon_{1}$ and $\sigma_{1}$ are the relative permittivity and conductivity of the ground respectively. The quasistatic approximation is valid for $\left|\gamma_{1}\right| \gg\left|\gamma_{0}\right|$ and $\left|\gamma_{0} \rho\right| \ll 1$. The abbreviations $I_{n}$ and $K_{n}$ are used for the modified Bessel functions of first and second kind of order $n$ with argument $\gamma_{1} \rho / 2$. The nonzero field components just above the interface in air due to an electric dipole above the interface with current moment $p$ are

$$
\begin{aligned}
E_{\rho}^{V E} & =\frac{-\gamma_{1}^{2} p}{2 \pi \sigma_{1} \rho} I_{1} K_{1} \\
E_{z}^{V E} & =\frac{-p}{2 \pi \sigma_{1} \rho^{3}} \frac{\gamma_{1}^{2}}{\gamma_{0}^{2}} \\
H_{\phi}^{V E} & =\frac{p}{2 \pi \rho^{2}} \\
E_{\rho}^{H E} & =\frac{p}{2 \pi \sigma_{1} \rho^{3}}\left[1+\left(1+\gamma_{1} \rho\right) e^{-\gamma_{1} \rho}\right] \cos \phi
\end{aligned}
$$




$$
\begin{aligned}
& E_{\phi}^{H E}=\frac{p}{2 \pi \sigma_{1} \rho^{3}}\left[2-\left(1+\gamma_{1} \rho\right) e^{-\gamma_{1} \rho}\right] \sin \phi \\
& E_{z}^{H E}=\frac{\gamma_{1}^{2} p}{2 \pi \sigma_{1} \rho} I_{1} K_{1} \cos \phi \\
& H_{\rho}^{H E}=\frac{p \sin \phi}{2 \pi \rho^{2}}\left[3 I_{1} K_{1}-\frac{\gamma_{1} \rho}{2}\left(I_{0} K_{1}-I_{1} K_{0}\right)\right] \\
& H_{\phi}^{H E}=\frac{-p \cos \phi}{2 \pi \rho^{2}} I_{1} K_{1} \\
& H_{z}^{H E}=\frac{p}{2 \pi \gamma_{1}^{2} \rho^{4}}\left[3-\left(3+3 \gamma_{1} \rho+\gamma_{1}^{2} \rho^{2}\right) e^{-\gamma_{1} \rho}\right] \sin \phi .
\end{aligned}
$$

For a magnetic dipole source with magnetic moment $m$ the fields are

$$
\begin{aligned}
& H_{\rho}^{V M}=\frac{-m}{4 \pi \rho^{3}}\left[16 I_{1} K_{1}+\gamma_{1}^{2} \rho^{2}\left(I_{1} K_{1}-I_{0} K_{0}\right)+4 \gamma_{1} \rho\left(I_{1} K_{0}-I_{0} K_{1}\right)\right] \\
& H_{z}^{V M}=\frac{-m}{2 \pi \gamma_{1}^{2} \rho^{5}}\left[9-\left(9+9 \gamma_{1} \rho+4 \gamma_{1}^{2} \rho^{2}+\gamma_{1}^{3} \rho^{3}\right) e^{-\gamma_{1} \rho}\right] \\
& E_{\phi}^{V M}=\frac{-m}{2 \pi \sigma_{1} \rho^{4}}\left[3-\left(3+3 \gamma_{1} \rho+\gamma_{1}^{2} \rho^{2}\right) e^{-\gamma_{1} \rho}\right] \\
& H_{\rho}^{H M}=\frac{m}{2 \pi \gamma_{1}^{2} \rho^{5}}\left[\left(\gamma_{1}^{3} \rho^{3}+5 \gamma_{1}^{2} \rho^{2}+12 \gamma_{1} \rho+12\right) e^{-\gamma_{1} \rho}-12+2 \gamma_{1}^{2} \rho^{2}\right] \cos \phi \\
& H_{\phi}^{H M}=\frac{m}{2 \pi \gamma_{1}^{2} \rho^{5}}\left[\left(\gamma_{1}^{2} \rho^{2}+3 \gamma_{1} \rho+3\right) e^{-\gamma_{1} \rho}+\gamma_{1}^{2} \rho^{2}-3\right] \sin \phi \\
& H_{z}^{H M}=\frac{m}{4 \pi \rho^{3}}\left[\gamma_{1}^{2} \rho^{2}\left(I_{1} K_{1}-I_{0} K_{0}\right)+4 \gamma_{1} \rho\left(I_{1} K_{0}-I_{0} K_{1}\right)+16 I_{1} K_{1}\right] \cos \phi \\
& E_{\rho}^{H M}=\frac{-\gamma_{1}^{2} m}{2 \pi \sigma_{1} \rho^{2}} I_{1} K_{1} \sin \phi \\
& E_{\phi}^{H M}=\frac{\gamma_{1}^{2} m}{2 \pi \sigma_{1} \rho^{2}}\left[3 I_{1} K_{1}-\frac{\gamma_{1} \rho}{2}\left(I_{0} K_{1}-I_{1} K_{0}\right)\right] \cos \phi \\
& E_{z}^{H M}=\frac{-\gamma_{1}^{2} m}{2 \pi \sigma_{1} \rho^{2}} \sin \phi .
\end{aligned}
$$

If the source is located just below the interface the above equations can be used with all components due to the vertical electric dipole (VE superscript) multiplied $\gamma_{0}^{2} / \gamma_{1}^{2}$. If the field evaluation point is just below the interface then all $E_{z}$ components should be multiplied by $\gamma_{0}^{2} / \gamma_{1}^{2}$.

Comparison of these equations with those used by Zablocki in [1] is complicated by differences in reference directions. Zablocki measures $\phi$ in a left hand sense about the $z$ axis. Also, his $z$ component of magnetic field is positive in the positive $z$ direction while his $z$ component of electric field is positive in the negative $z$ direction. Taking these differences into account, it appears that there are sign errors in Zablocki's equations (6), (7), (14), (15), (20), (21), (23) and (25). While Zablocki's equations for $H_{\rho}^{V M}$ and $H_{z}^{H M}$ are correct, his plotted results appear to be missing a factor of two. 


\section{Results for Unit Dipole Sources}

Plots of the normalized field magnitudes from the quasistatic equations are shown in Figs. 2 and 3 for a buried source with the field evaluated in air. The components $E_{\rho}^{V E}$, $E_{z}^{H E}$ and $E_{z}^{H M}$ decrease as $\rho^{2}$ and so that their normalized forms increase with $\rho$. The quasistatic results were found to agree with the numerical evaluation of the Sommerfeld integrals to within a relative error of $10^{-3}$ to $10^{-4}$. The noisiness of this error indicated that the numerical evaluation was the less accurate of the two.

Of the candidate mechanisms for generation of ELF radiation by a nuclear event the magnetic dipole due to the Earth's magnetic field and emplacement pipe currents have known orientations. The emplacement pipe currents represent a vertical electric current source for which the fields can be found in Figs. 2 and 3. The exclusion of the Earth's magnetic field would produce a magnetic dipole source opposing the Earth's field which has an inclination of about 60 degrees at the Nevada Test Site. Hence the magnetic dipole is oriented toward the South with an elevation angle of 60 degrees. The field components to the North, South and East or West of such a dipole with unit strength are plotted in Figs. 4 and 5. Similar plots presented by Zablocki differ from these, apparently due to sign errors in [1].

All of the preceeding field values have been computed for sources located just below the surface of the ground with the evaluation point just above the ground in air. The effect of attenuation from events occurring at typical depths of several hundred meters is illustrated in Fig. 6 where the field components of a magnetic dipole at height $z_{s}$ are shown normalized to the values for $z_{s}=0$. The ground conductivity was taken as $\sigma_{1}=0.01 \mathrm{~S} / \mathrm{m}$ with $\rho=7\left(10^{3}\right) \mathrm{m}$ and a frequency of $10 \mathrm{~Hz}$. These results were obtained by numerical evaluation of the Sommerfeld integrals, since depth does not appear in the quasistatic formulas. The attenuation of the field components is seen to be similar to or less than the far field attenuation factor $\exp \left(-\gamma_{1} z_{s}\right)$, depending on the near field behavior of the particular component. Effects of similar order would be seen in the phase. At typical depths of about 200 meters it appears reasonable to neglect the effect of depth in a first order analysis of ELF radiation.

The field components at a fixed distance of $\rho=7\left(10^{3}\right) \mathrm{m}$ are shown as a function of frequency in Fig. 7 for a magnetic dipole and in Fig. 8 for an electric dipole source with ground conductivity $\sigma_{1}=0.01 \mathrm{~S} / \mathrm{m}$. Each field component is shown for the value of $\phi$ for which it is a maximum. The field values were computed in increments of $1 \mathrm{~Hz}$ to a frequency of $256 \mathrm{~Hz}$. These results were then used to obtain the transient response for a step function excitation by computing the inverse FFT to get the impulse response and then integrating to obtain the step function response. These calculations were performed with the signal processing code SIG developed at LLNL. The field components that tend toward zero with increasing frequency show a finite rise time in the step response, while the components that approach a constant value at high frequency show a rise time of essentially zero, less than the resolution of the plot. Of course the measured transient field would depend on the frequency response of the measuring equipment which is generally less than $256 \mathrm{~Hz}$. This is particularly true for the components, such as $E_{z}^{H M}$, for which the 
field strength continues to increase with increasing frequency. The step response is then dominated by ringing at the highest frequency in the band. Filtering could be applied in the $S I G$ processing if the appropriate characteristic were known. For a source at a depth of several hundred meters the increasing attenuation with frequency would also have a significant effect on the higher frequencies and resulting transient responses.

\section{Conclusion}

The field components due to point dipole sources in the ground have been evaluated for distances typical of ELF measurement stations near to ground zero. We did not have sufficient time for a through comparison of these results with measured signals. However, there does not appear to be any obvious correlation between the measured signals and computed fields of these simple sources. Hence it may be necessary to consider more complicated models involving a combination of sources and to take into account effects on the signal propagation to the measurement site.

Several computer codes have been developed at LLNL for analyzing complex electromagnetic models. These include the Numerical Electromagnetics Code (NEC) which can model wires in or near the surface of the earth through a method-of-moments solution of an integral equation, and a finite difference time domain code (FDTD) which integrates Maxwell's equations in an arbitrary medium. Both of these codes are generally used to compute induced currents or scattered fields due to an exciting field or voltage source. Such an approach in this case would require an evaluation of the physical processes involved in exciting the currents. This difficult step might be skipped if currents could be measured at one or more points in the ground or on emplacement pipes. The current distributions could then be approximated over the electrically small regions of interest and the problem reduced to determining the fields due to a combination of the point-source fields discussed above. A code such as NEC could be used to determine the current distribution on an emplacement pipe, given the current at one point, if the distribution could not be estimated with sufficient accuracy. However, NEC can become inaccurate at very low frequencies, particularly for models involving an interface or wire loops. Hence it might be necessary start at a higher frequency and determine the limiting form of the current for decreasing frequency.

Electromagnetic modeling codes could also be used to analyze the propagation of the signal to the measurement site and possible interactions of the measuring antennas and nearby equipment. An alternate approach might be to calibrate the measuring equipment with known sources at ground zero.

The codes that produced the above field plots can easily be modified to plot the fields due to a combination of dipole sources or the transient signals measured for given source and receiver characteristics. A coordinated effort of measurement and modeling could be helpful in understanding this problem. 


\section{REFERENCES}

[1] C. J. Zablocki, "Electrical Transients Observed during Underground Nuclear Explosions," Journal of Geophysical Research, Vol. 71, No. 14, pp. 3523-3542, July 15, 1966.

[2] G. J. Burke, C. G. Dease, E. M. Didwall and R. J. Lytle, Numerical Modeling of Subsurface Communication, Lawrence Livermore National Laboratory, Rept. UCID20439 Rev. 1, August 1985.

[3] M. B. Kraichman, Handbook of Electromagnetic Propagation in Conducting Media, U. S. Government Printing Office, Washington D. C., 1970. 

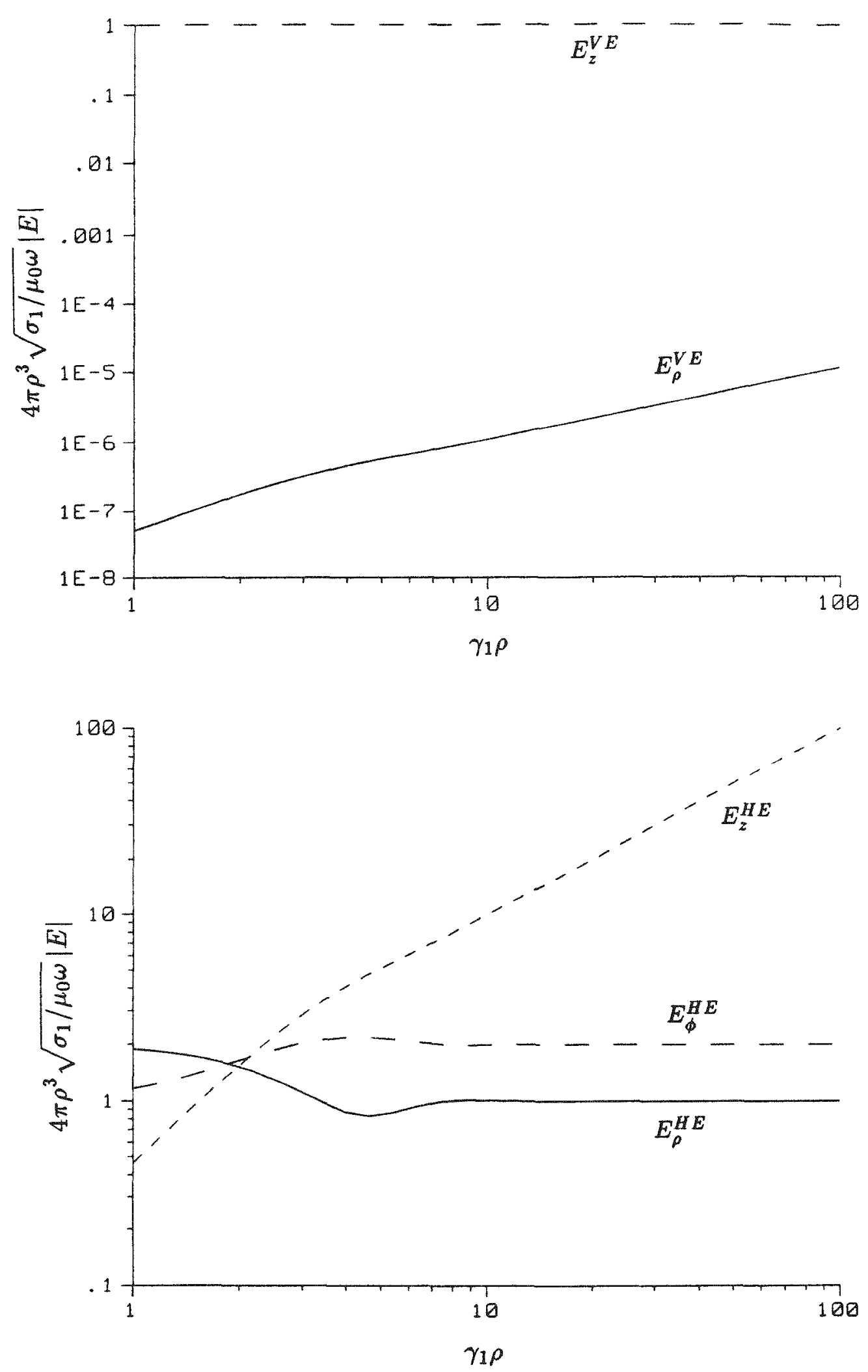

Fig. 2. Field components due to an electric dipole source with unit current moment. The source is just below the interface and the field is evaluated just above the interface in air. 


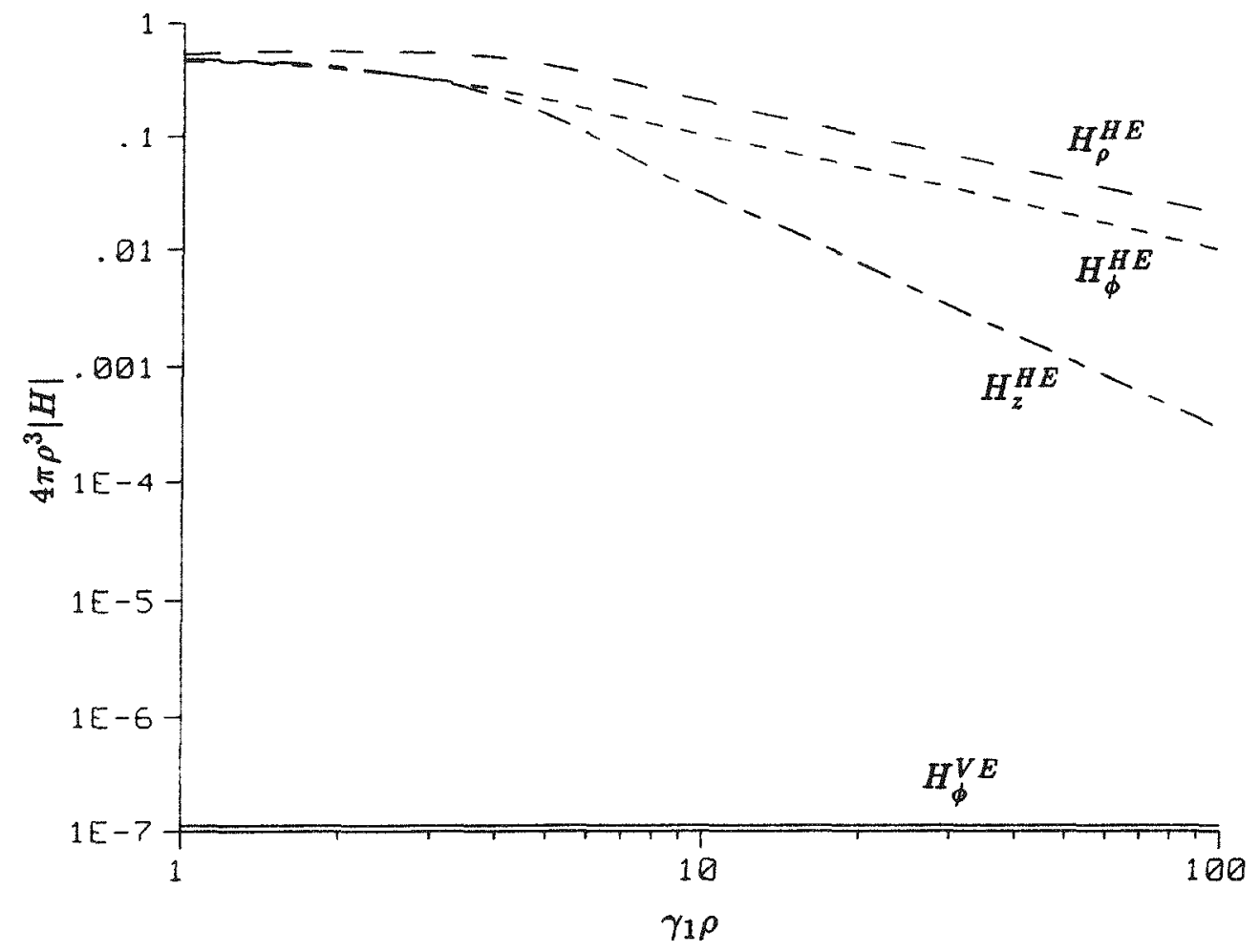

Fig. 2. Cont.

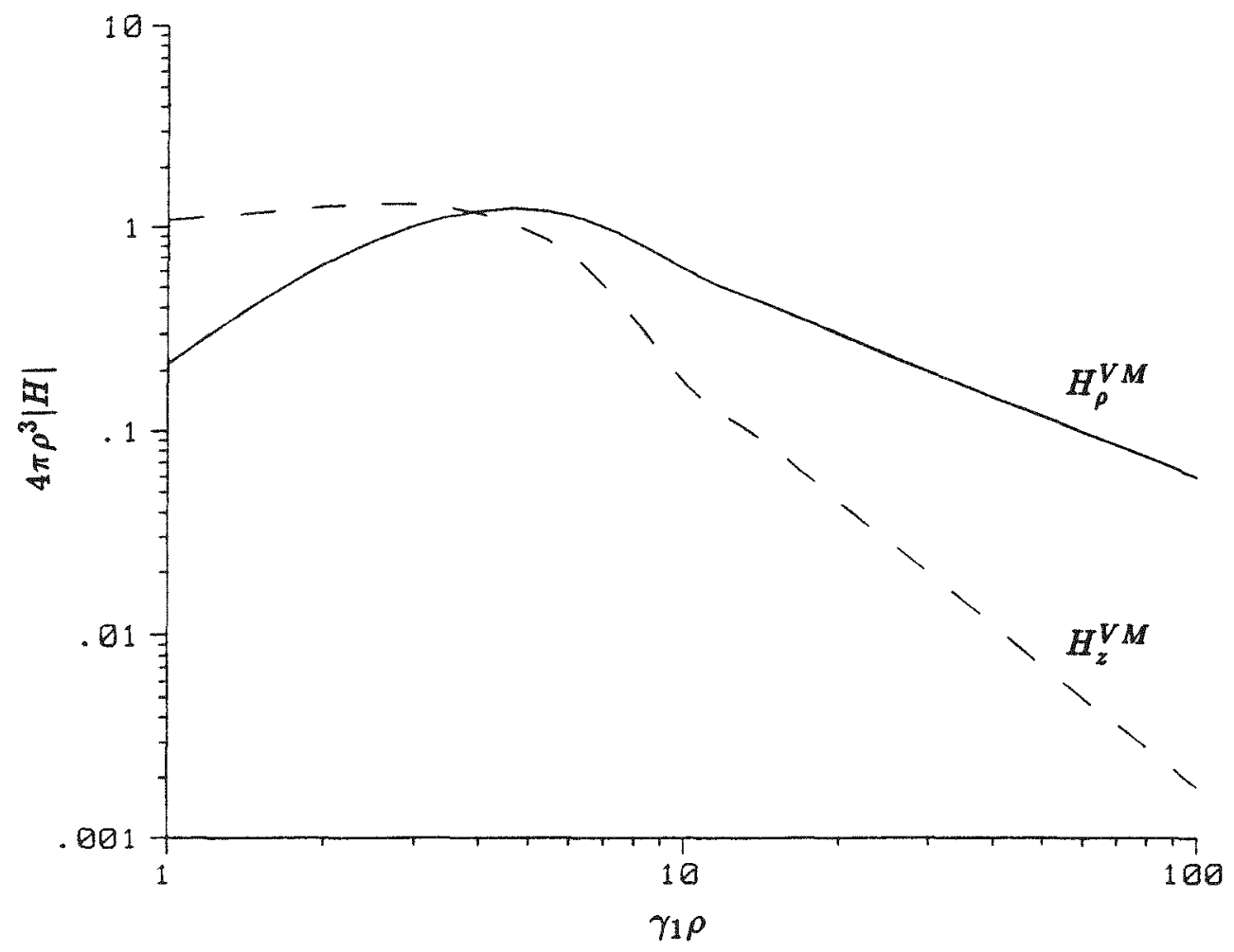

Fig. 3. Field components due to a magnetic dipole source with unit magnetic moment. The field is evaluated just above the interface in air. 

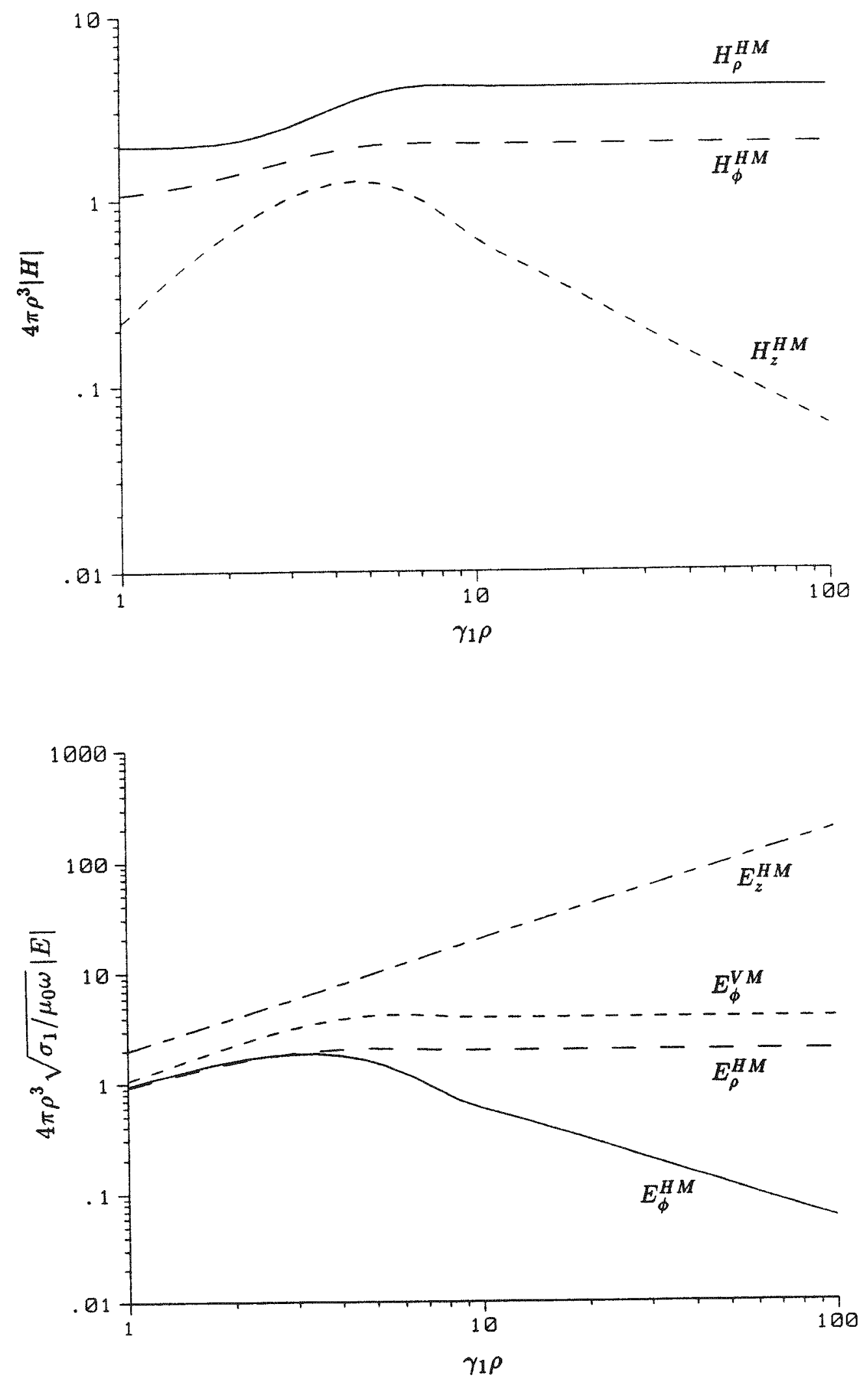

Fig. 3. Cont. 

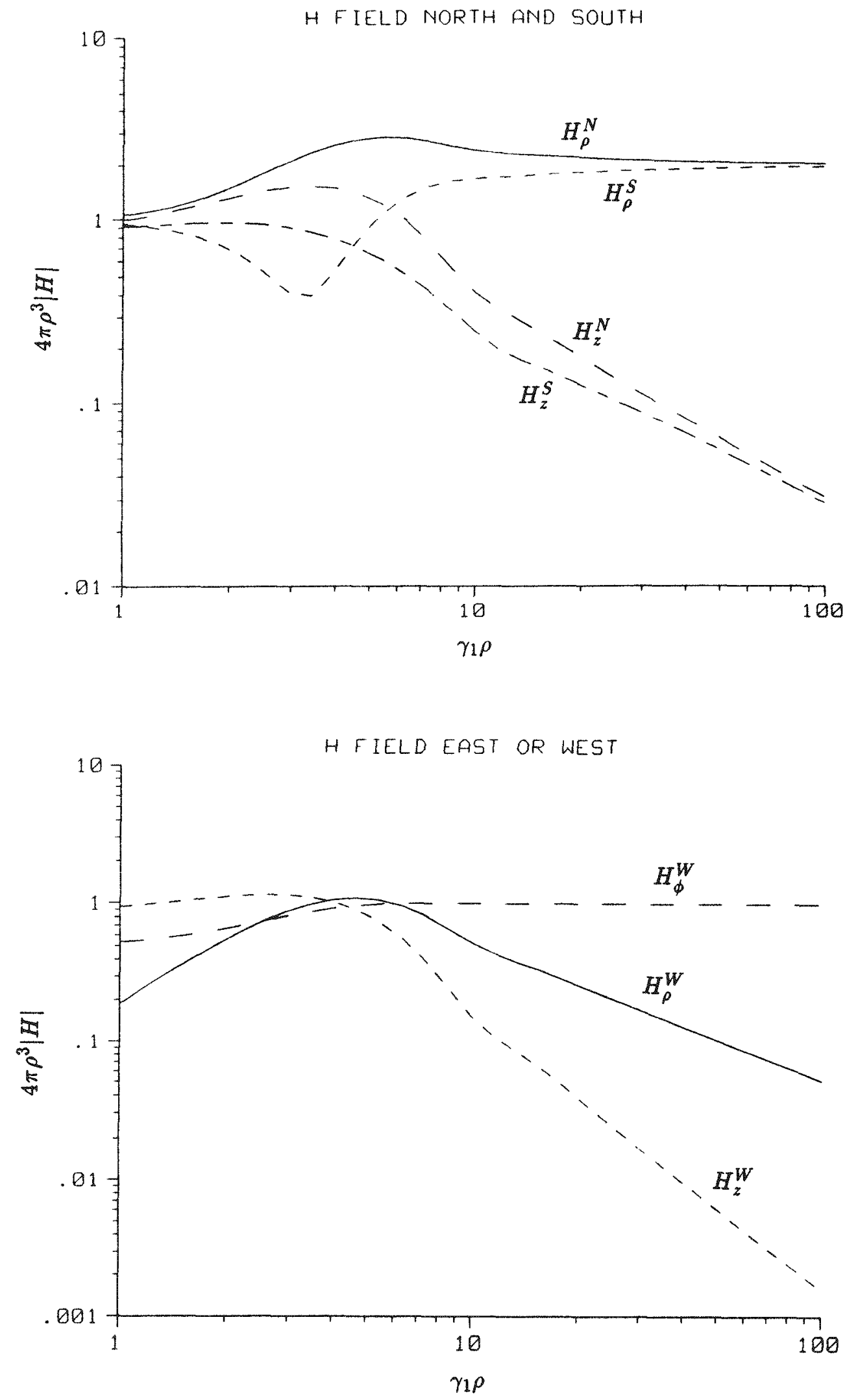

Fig. 4. Magnetic field components North, South and West of a unit magnetic dipole parallel to the Earth's magnetic field. 

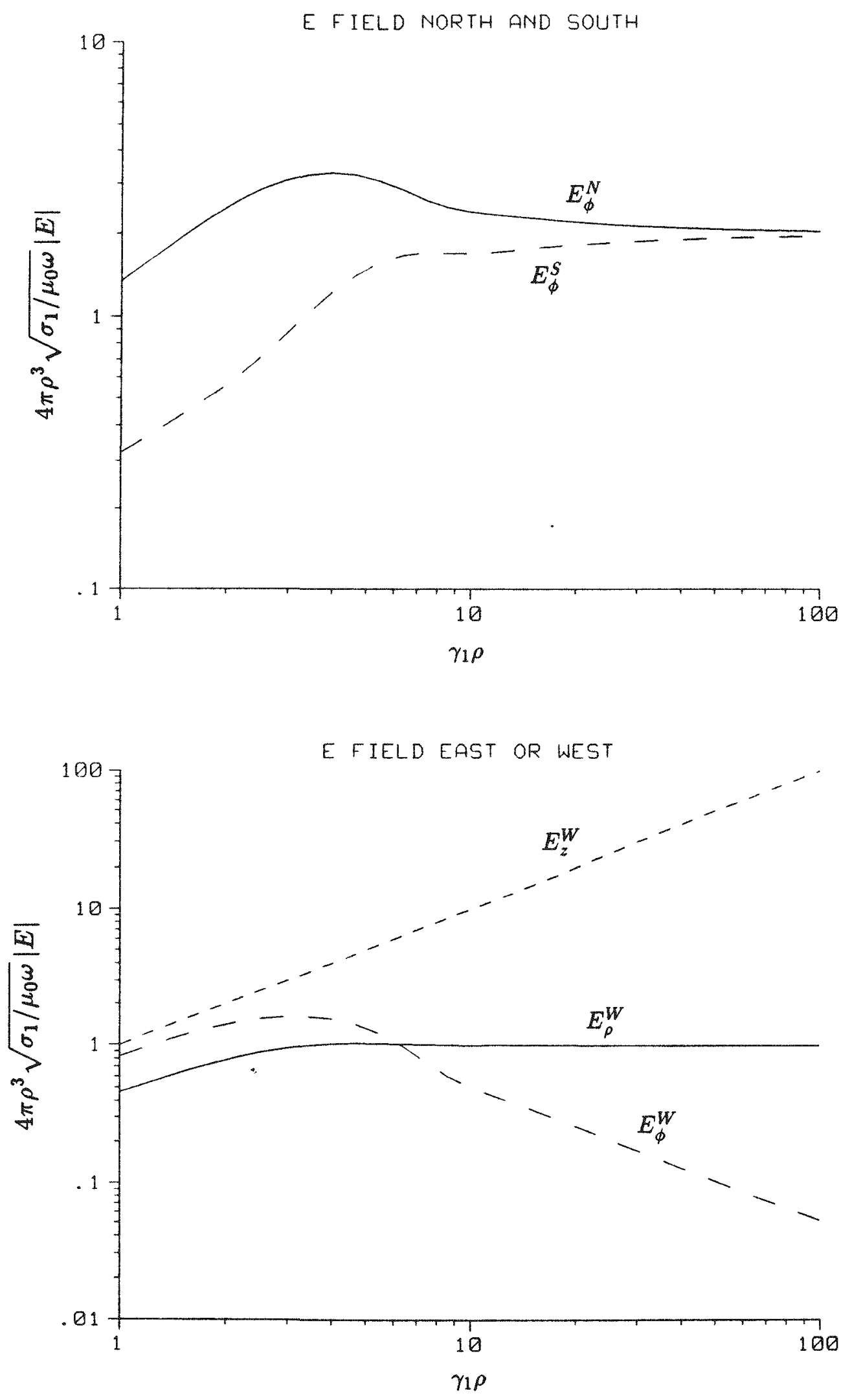

Fig. 5. Electric field components North, South and West of a unit magnetic dipole parallel to the Earth's magnetic field. 

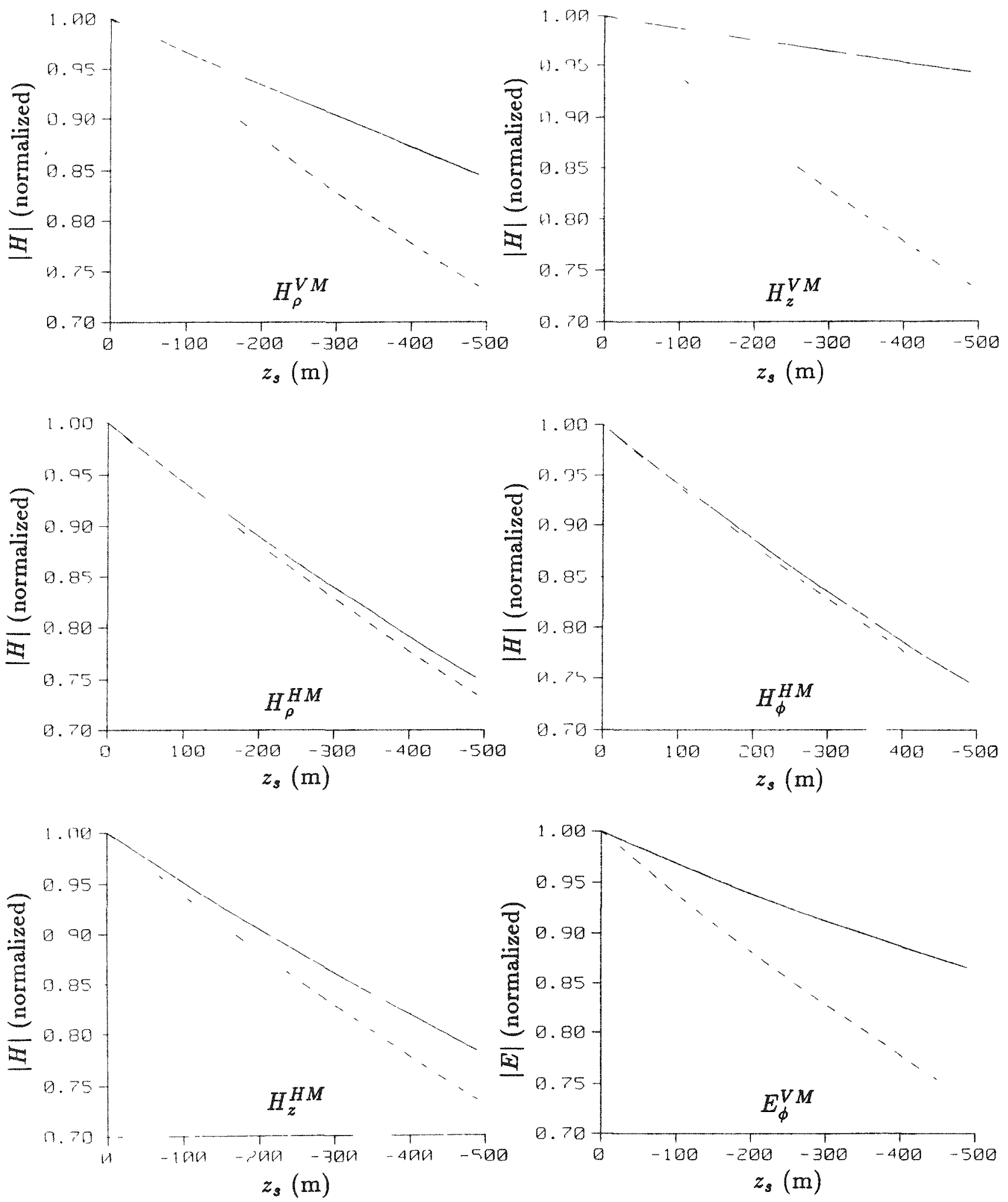

Fig. 6. Normalized field components due to a magnetic dipole at height $z_{3}$ in the Earth with $\rho=7\left(10^{3}\right) \mathrm{m}$, $\sigma_{1}=0.01 \mathrm{~S} / \mathrm{m}$, and frequency $=10 \mathrm{~Hz}$. The dashed line represents $\exp \left(-\gamma_{1} z_{3}\right)$. 

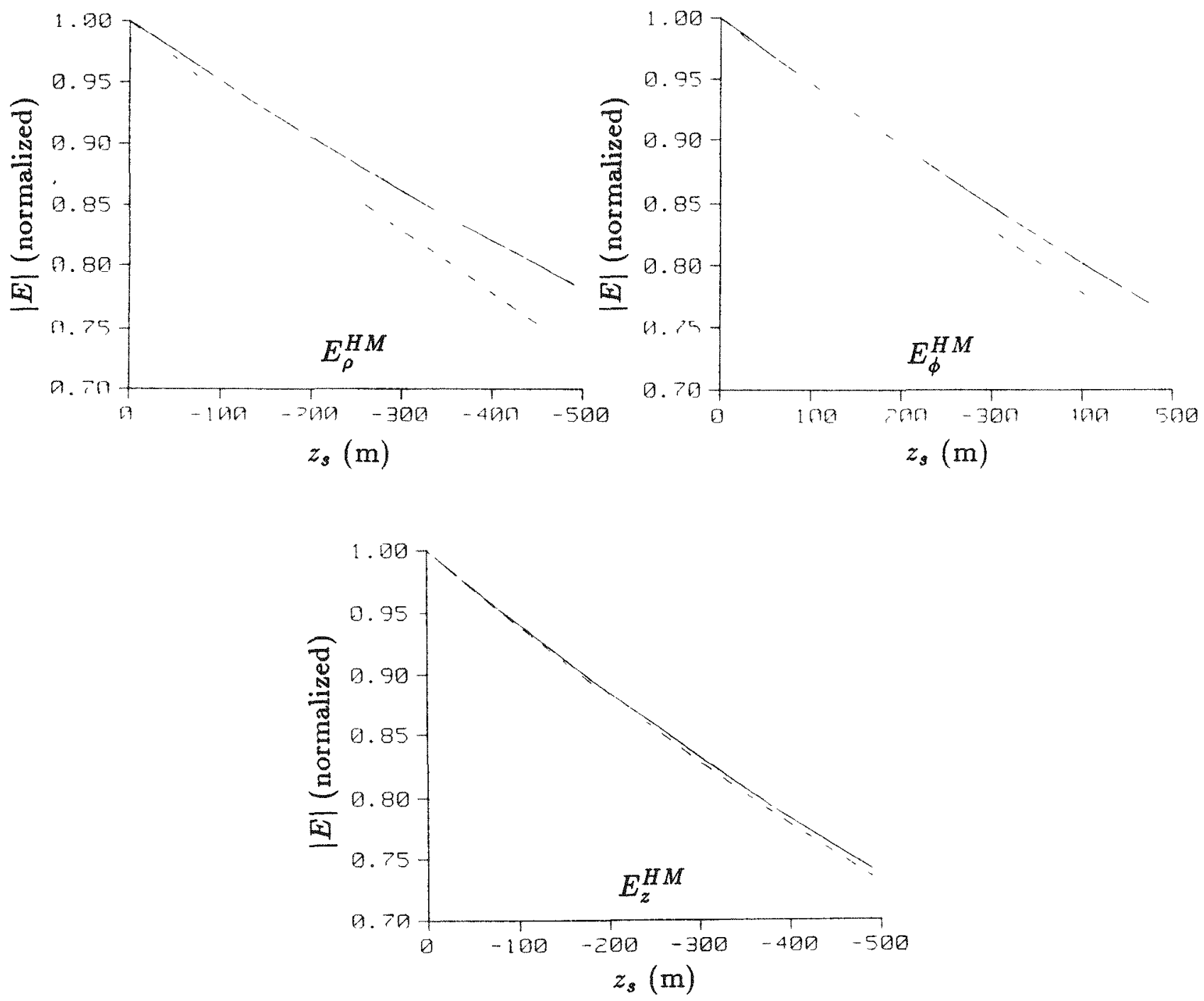

Fig. 6. Cont. 

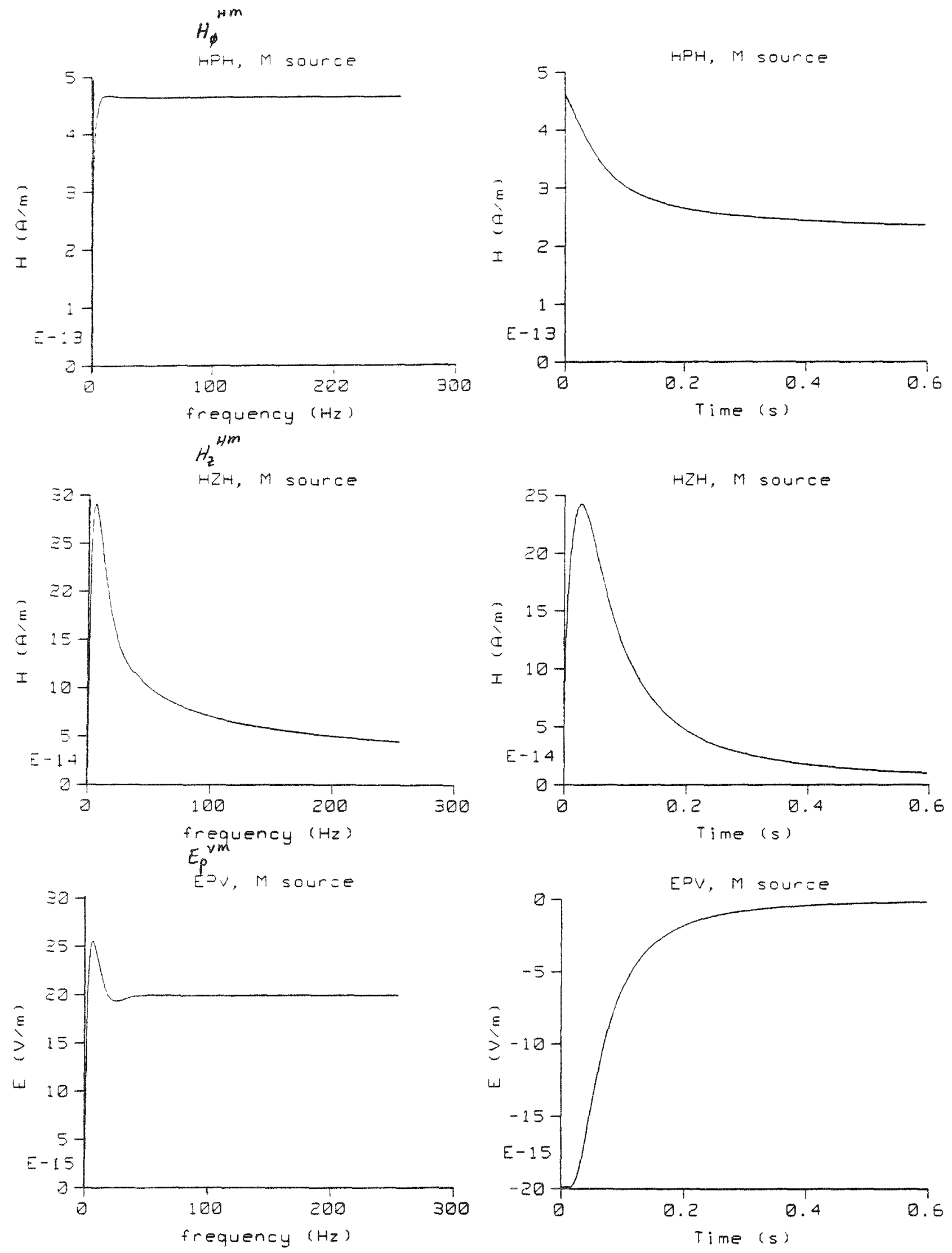

Fig. 7. Field components in air due to a unit magnetic dipole source in the Earth for $\rho=7\left(10^{3}\right) \mathrm{m}$ and $\sigma_{1}=0.01 \mathrm{~S} / \mathrm{m}$. The magnitude of the field as a function of frequency and the transient response to a step function source are shown. 

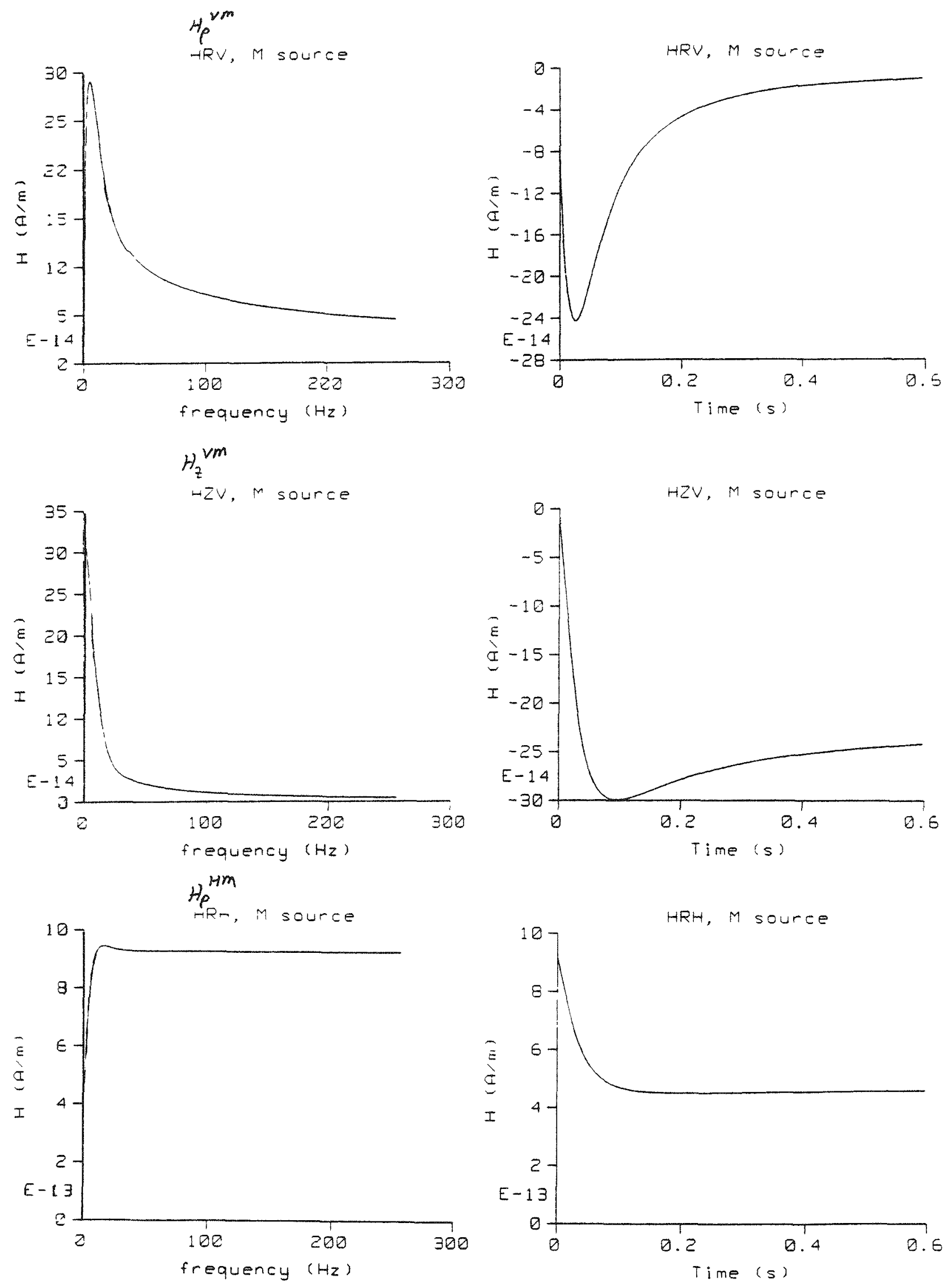

Fig. 7. Cont. 

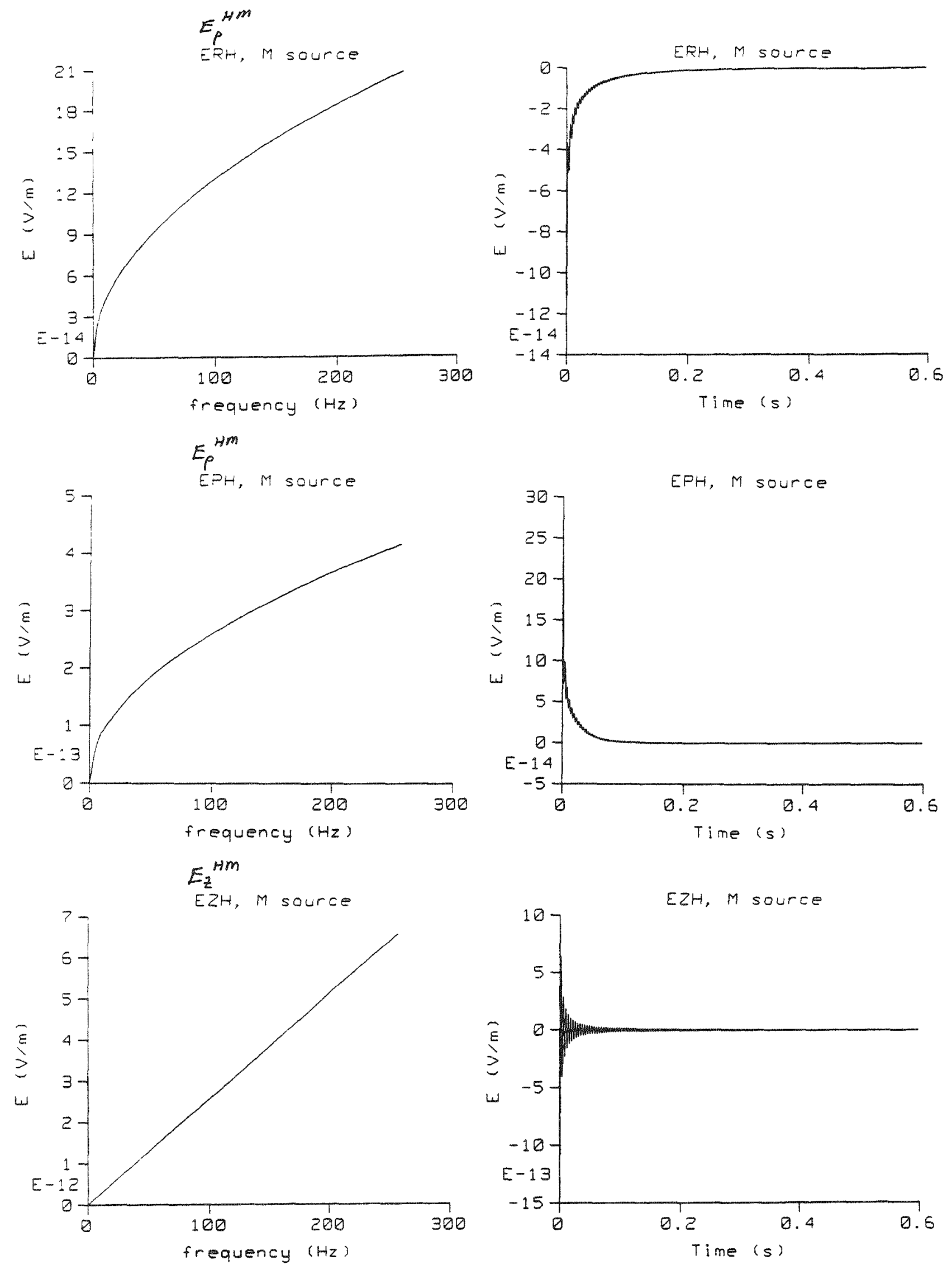

Fig. 7. Cont. 

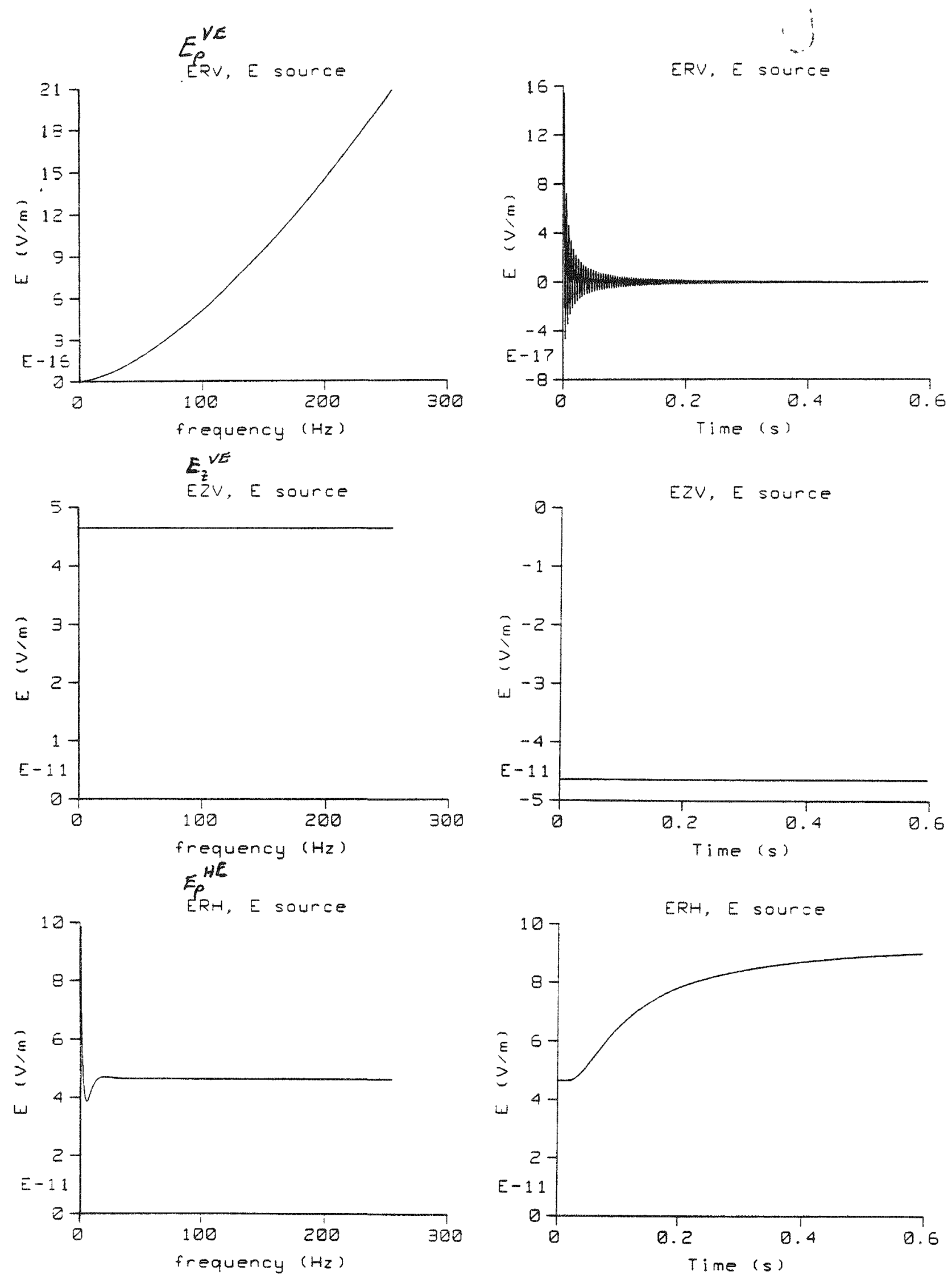

Fig. 8. Field components in air due to a unit electric dipole source in the Earth for $\rho=7\left(10^{3}\right) \mathrm{m}$ and $\sigma_{\mathrm{l}}=0.01 \mathrm{~S} / \mathrm{m}$. The magnitude of the field as a function of frequency and the transient response to a step function source are shown. 

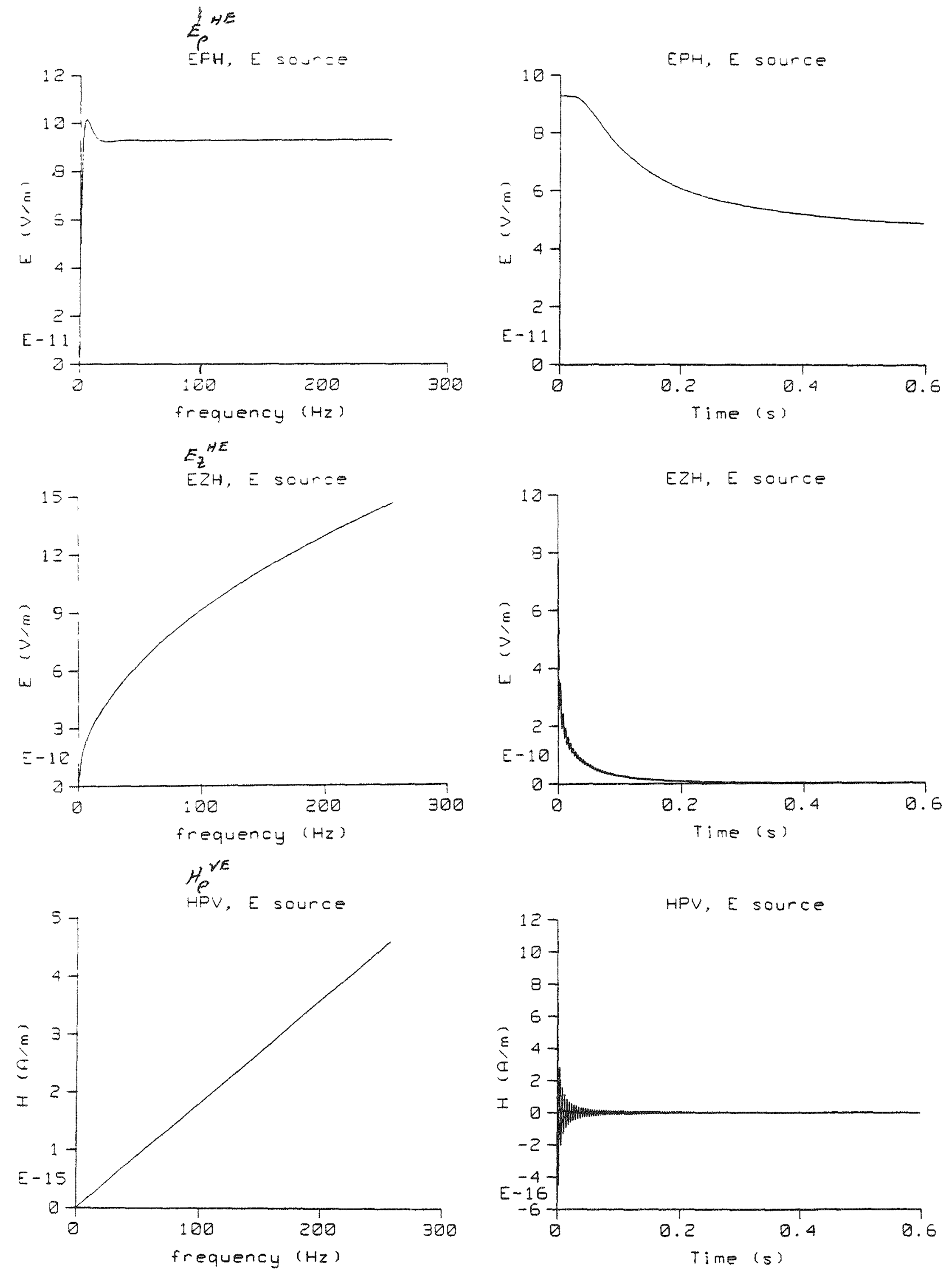

Fig. 8. Cont. 

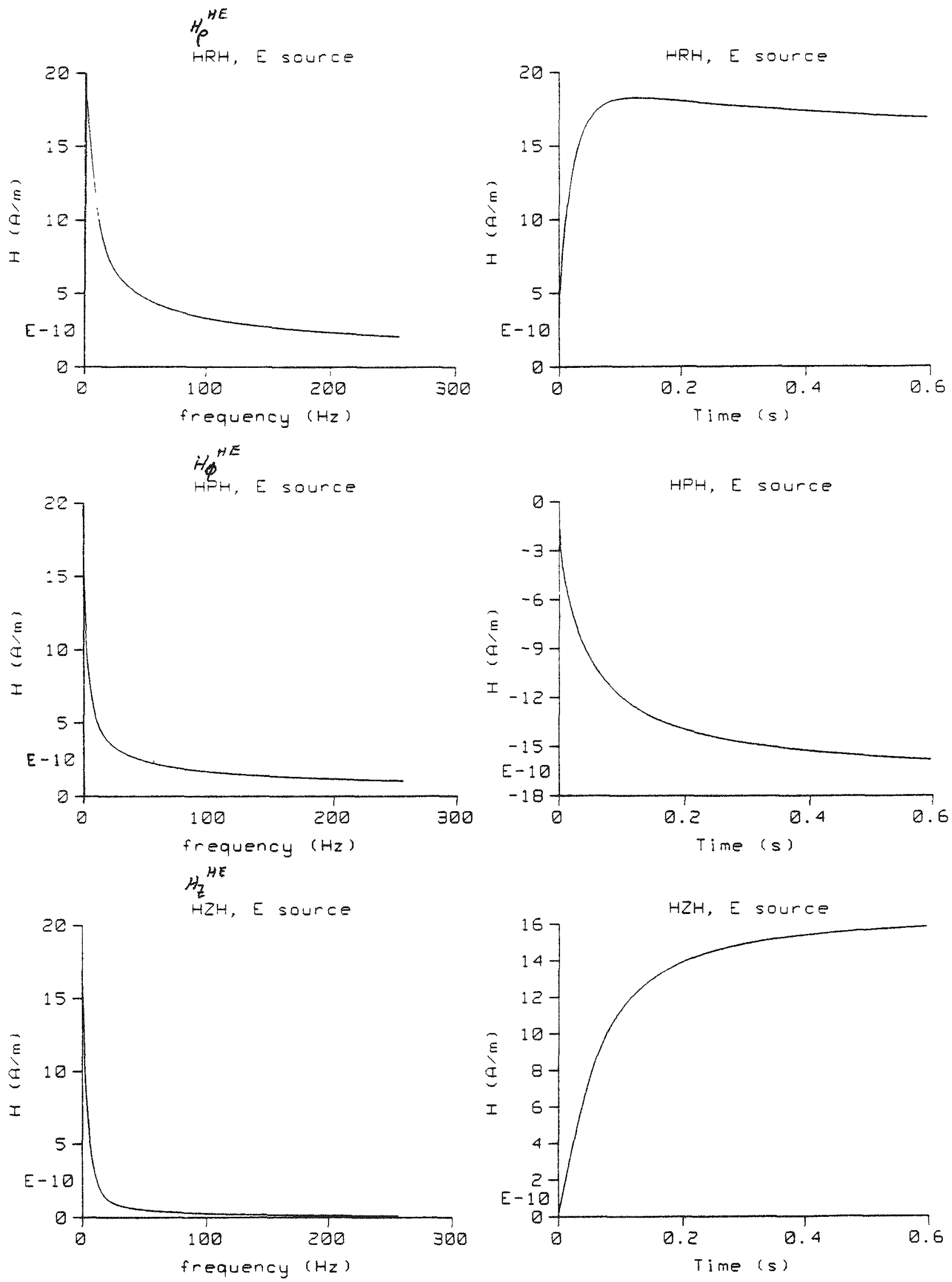

Fig. 8. Cont. 


\title{
Appendix B
}

\section{Excitation of the Earth-Ionosphere Cavity by an Underground Nuclear Test}

A Theoretical Analysis of Recent Results at the Nevada Test Site

\author{
D. D. Sentman \\ Institute for Geophysics and Planetary Physics \\ University of California \\ Los Angeles, California 90024-1567
}




\section{Introduction}

Recent six-axis measurements of the prompt ELF electric and magnetic near fields in the vicinity of underground tests at the Nevada Test Site have shown that the detonation generates an impulse of several hundred $\mathrm{ms}$ duration. The experimental apparatus and the results of these measurements are reported separately. One of the goals of these measurements was to determine if the impulsive currents generated by the underground test could couple sufficient ELF wave energy into the earth-ionosphere cavity to be detected at great distances from the test. Such a capability would permit an independent remote detection capability to augment the seismic detection methods that form the primary technical means for monitoring compliance with the test ban treaty.

In this report we provide a retrospective interpretation of the absence at distant locations of detectable signals that were observed near the underground test. We review the theoretical techniques for determining the excitation efficiency of a hypothetical excitation source for the earth-ionosphere cavity. In addition to the case of impulsive excitation by lightning treated in the literature, we discuss the case of long-duration current transients implied by the near-field observations.

\section{Theory}

Several authors have dealt with the technique of calculating the strength of ELF electromagnetic signals generated by current sources within the earthionosphere cavity (e.g., Wait, 1962; Galejs, 1972; Bliokh et al., 1980; Polk, 1982). In these works the emphasis is on the power spectrum of the normal modes within the cavity. Good agreement has been achieved between theoretical estimates of the power spectrum of ELF waves in the earth-ionosphere cavity and those observed experimentally. Since the spectrum is highly nonstationary, however, averaging over many minutes has been required to obtain a power spectrum that displays the normal mode eigenfrequencies of the cavity.

For the present case we are interested in the time domain response of the earth-ionosphere cavity to a single impulsive excitation of limited duration and how this response compares to that of lightning constituting the principal source of background noise at ELF. We shall derive the temporal signatures of current sources for the case of an ideal resonator with no losses. The case of the real, lossy cavity will then be treated as a correction to the ideal case using known 
values of the eigenfrequencies in the earth-ionosphere cavity. We shall closely follow the approach and notation of Bliokh et al. (1980).

Assuming that all quantities vary as $e^{i \omega t}$, in SI units Faraday's and Ampere's laws are, respectively,

$$
\begin{gathered}
\nabla \times \mathrm{E}=-i \omega \mu_{o} \mathrm{H}, \\
\nabla \times \mathrm{H}=i \omega \epsilon_{o} \mathrm{E}+\mathrm{J},
\end{gathered}
$$

where $\mathrm{E}=\mathrm{E}(\mathrm{r}, \omega)$ is the electric field and $\mathrm{H}=\mathrm{H}(\mathrm{r}, \omega)$ is the magnetic intensity. $\mathrm{J}=\mathrm{J}(\mathrm{r}, \omega)$ is the external source current is normally lightning but in general can be any driving current.

Faraday's and Ampere's laws for the $n^{\prime} t h$ normal mode fields denoted by $\mathrm{E}_{\mathrm{n}}$ and $H_{n}$ may be written

$$
\begin{gathered}
\nabla \times \mathrm{E}_{\mathrm{n}}=-i \omega_{n} \mu_{o} \mathrm{H}_{\mathrm{n}}, \\
\nabla \times \mathrm{H}_{\mathrm{n}}=i \omega_{n} \epsilon_{o} \mathrm{E}_{\mathrm{n}},
\end{gathered}
$$

where we have assumed that the eigenfrequencies $\omega_{n}$ are real. Using the identity of the divergence of a vector cross product, we may write

$$
\begin{gathered}
\nabla \cdot\left(\mathrm{E} \times \mathrm{H}_{\mathrm{n}}^{*}\right)=-i \omega \mu_{o} \mathrm{H} \cdot \mathrm{H}_{\mathrm{n}}^{*}+i \omega_{n} \epsilon_{o} \mathrm{E} \cdot \mathrm{E}_{\mathrm{n}}^{*}, \\
\nabla \cdot\left(\mathrm{E}_{\mathrm{n}}^{*} \cdot \mathrm{H}\right)=i \omega_{n} \mu_{o} \mathrm{H} \cdot \mathrm{H}_{\mathrm{n}}^{*}-i \omega \epsilon_{o} \mathrm{E} \cdot \mathrm{E}_{\mathrm{n}}^{*}-\mathrm{E}_{\mathrm{n}}^{*} \cdot J .
\end{gathered}
$$

Integrating (5) and (6) over the volume of the earth-ionosphere cavity, making use of Gauss' law and taking into account that the electric field is normal to the surface of a perfectly conducting earth and falls to zero faster than $1 / r^{2}$ at very large heights, we get

$$
\begin{gathered}
-i \omega \int_{V} \mu_{0} \mathrm{H} \cdot \mathrm{H}_{\mathrm{n}}^{*} d V+i \omega_{n} \int_{V} \epsilon_{0} \mathrm{E} \cdot \mathrm{E}_{\mathrm{n}}^{*} d V=0, \\
i \omega_{n} \int_{V} \mu_{o} \mathrm{H} \cdot \mathrm{H}_{\mathrm{n}}^{*} d V-i \omega \int_{V} \epsilon_{o} \mathrm{E} \cdot \mathrm{E}_{\mathrm{n}}^{*} d V-\int_{V} \mathrm{E}_{\mathrm{n}}^{*} \cdot \mathrm{J} d V=0 .
\end{gathered}
$$

Assuming that the fields $\mathrm{E}$ and $\mathrm{H}$ may be represented as an expansion over the normal modes of the cavity, viz.

$$
\mathrm{H}=\sum_{\mathrm{m}} \alpha_{\mathrm{m}} \mathrm{H}_{\mathrm{m}}, \quad \mathrm{E}=\sum_{\mathrm{m}} \beta_{\mathrm{m}} \mathrm{E}_{\mathrm{m}},
$$


where the sum is taken over all normal modes, expressions (7) and (8) become

$$
\begin{gathered}
-\omega \sum_{\mathbf{n}} \alpha_{\mathrm{n}} \int_{V} \mu_{o} \mathrm{H}_{\mathrm{m}} \cdot \mathrm{H}_{\mathrm{n}}^{*} d V+\omega_{n} \sum_{\mathbf{n}} \beta_{\mathrm{n}} \int_{V} \epsilon_{o} \mathrm{E}_{\mathrm{m}} \cdot \mathrm{E}_{\mathrm{n}}^{*} d V=0 \\
\omega_{n} \sum_{\mathbf{n}} \alpha_{\mathrm{n}} \int_{V} \mu_{o} \mathrm{H}_{\mathrm{m}} \cdot \mathrm{H}_{\mathrm{n}}^{*} d V-\omega \sum_{\mathbf{n}} \beta_{\mathrm{n}} \int_{V} \epsilon_{o} \mathrm{E}_{\mathrm{m}} \cdot \mathrm{E}_{\mathrm{n}}^{*} d V+i \int_{V} \mathrm{E}_{\mathrm{n}}^{*} \cdot \mathrm{J} d V=(10.1)
\end{gathered}
$$

The eigenmodes are orthogonal for a spherically symmetric cavity, so we take as normalization (Bliokh et al., 1980)

$$
\int_{V} \mu_{o} \mathrm{H}_{\mathrm{m}} \cdot \mathrm{H}_{\mathrm{n}}^{*} d V=\int_{V} \epsilon_{o} \mathrm{E}_{\mathrm{m}} \cdot \mathrm{E}_{\mathrm{n}}^{*} d V=K \delta_{n, m} .
$$

Defining

$$
\mathcal{J}_{\mathrm{n}}=\frac{1}{K} \int_{V} \mathrm{E}_{\mathrm{n}}^{*} \cdot J d V
$$

and using the normalization (11) yields a set of linear equations for the expansion coefficients $\alpha_{n}$ and $\beta_{n}$

$$
\begin{gathered}
\omega \alpha_{n}-\omega_{n} \beta_{n}=0, \\
\omega_{n} \alpha_{n}-\omega \beta_{n}=-i \mathcal{J}_{\mathbf{n}},
\end{gathered}
$$

which yield for the expansion coefficients

$$
\alpha_{\mathrm{n}}=\frac{i \omega \mathcal{J}_{\mathrm{n}}}{\omega^{2}-\omega_{n}^{2}}, \quad \beta_{\mathrm{n}}=\frac{i \omega_{n} \mathcal{J}_{\mathrm{n}}}{\omega^{2}-\omega_{n}^{2}}
$$

A common source of excitation of the earth-ionosphere cavity is cloud-toground lightning. Vertical lightning is a much more efficient exciter of the cavity than is horizontal lightning, as evidenced by the fact that $\mathcal{J}_{\mathbf{n}}$ in expression (13) is the projection of the source current $J$ onto the normal mode electric field $E_{n}$. At the surface of the earth this electric field component is vertical, thereby favoring excitation by vertical lightning over horizontal lightning. More generally, vertical current dipoles of any kind are more efficient sources of cavity excitation than are horizontal current dipoles. 
To compute the excitation amplitudes of the cavity from a vertical source we model the current located at the pole in a spherical coordinate system as an impulse of initial amplitude $I_{o}$, channel length $d s$ and exponential decay time $T$

$$
J(\mathrm{r}, \mathrm{t})= \begin{cases}\frac{I_{0} d s e^{-t / T}}{4 \pi r^{2} \sin \theta} \delta(\theta), & a<r<a+d s, t>0, \\ 0 & r<a, r>a+d s, t<0 .\end{cases}
$$

where $a$ is the radius of the earth. The Fourier transform of the current is

$$
J(\mathrm{r}, \omega)=\frac{I_{o} d s T}{4 \pi r^{2} \sin \theta(1+i \omega T)} \delta(\theta) .
$$

Putting this into the expression for $\mathcal{J}_{\mathrm{n}}$ yields

$$
\mathcal{J}_{\mathrm{n}}=\frac{I_{o} d s T}{K(1+i \omega T)} \mathrm{E}_{\mathrm{n}}(\theta=0) .
$$

The angular dependence of the normal modes in a loss cavity is described by the spherical harmonics $Y_{p q}(\theta, \phi)$. In the case of a spherically symmetric cavity and a vertical source there is no excitation of modes for which $p \neq q$, and thus no longitudinal dependence. The angular dependence in this case can be described solely in terms of Legendre polynomials. Combining (16) and (17) and taking into account the normalization of the spherical harmonics, we arrive at an expression for the electric field intensity similar to that given by Bliokh et al. (1980), but with a sign correction consistent with that required to have the Poynting flux directed away from the source region

$$
E(\omega, \theta)=\frac{i I_{o} d s}{4 \pi a^{2} h \epsilon_{o}} \sum_{n=0}^{\infty}(2 n+1) P_{n}(\cos \theta) \frac{\omega T}{\left(\omega^{2}-\omega_{n}^{2}\right)(1+i \omega T)}
$$

To determine the temporal response corresponding to the Fourier expression (20) for the electric field, we perform an inverse transform

$$
E(t)=\frac{1}{2 \pi} \int_{-\infty}^{+\infty} E(\omega) e^{i \omega t} d \omega
$$




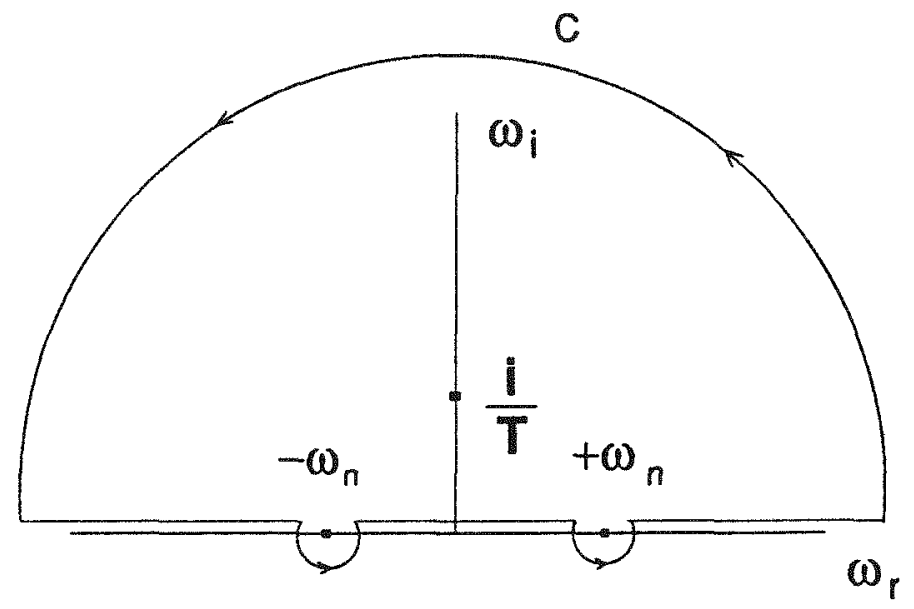

Figure 1 Contour used to evaluate expression (20). The three simple poles are located at $\omega=+\omega_{n}$, $\omega=-\omega_{n}$ and $\omega=i / T$. The contour is closed at infinity around $\mathbb{C}$ in the upper half plane.

The integrand of (20) has three simple poles, at $\omega= \pm \omega_{n}$ and $\omega=+i / T$. The integration may be performed around the counterclockwise contour $\mathrm{C}$ shown in Figure 1.

The residues of $\omega /\left(\omega^{2}-\omega_{n}^{2}\right)(1+i \omega T)$ are

$$
\begin{gathered}
\mathcal{R}\left(\omega=\omega_{n}\right)=\frac{e^{-\gamma_{n} t} e^{i \omega_{n} t}}{2\left(1+i \omega_{n} T\right)}, \\
\mathcal{R}\left(\omega=-\omega_{n}\right)=\frac{e^{-\gamma_{n} t} e^{-i \omega_{n} t}}{2\left(1-i \omega_{n} T\right)}, \\
\mathcal{R}(\omega=i / T)=\frac{-e^{-t / T}}{1+\omega_{n}^{2} T^{2}} .
\end{gathered}
$$

where now we have separated out the small imaginary parts $\gamma_{n}=\operatorname{Im}\left(\omega_{n}\right)$ of the eigenfrequencies and made use of the fact that for real fields the complex Fourier coefficients are Hermitian. For a dissipative ionosphere $\gamma_{n}$ is positive. The expression for the electric field becomes, finally,

$$
\begin{aligned}
E(\theta, t)= & \frac{I_{o} d s T}{4 \pi a^{2} h \epsilon_{o}} \sum_{n=0}^{\infty}(2 n+1) P_{n}(\cos \theta) \frac{1}{\left(1+\omega_{n}^{2} T^{2}\right)} \\
& \times\left\{e^{-t / T}-e^{-\gamma_{n} t}\left[\cos \left(\omega_{n} t\right)+\omega_{n} T \sin \left(\omega_{n} t\right)\right]\right\} .
\end{aligned}
$$


The first term in the braces expresses the direct, non-resonant contribution to the field from the lightning current with a polarity and characteristic decay time equal to that of the source. The terms inside the square brackets express the resonant behavior of the cavity. The first of these terms describes the impulse response, and the second expresses the driving effect from a source that persists over a non-negligible interval of the wave period. After excitation the resonant terms exponentially damp out at their own individual rates. Note that the polarity of the resonant part of the electric field at $t=0$ is opposite that of the source. This may be interpreted as follows. A vertically upward directed current corresponds to an increase in the electric dipole moment. This can be accomplished either by raising positive charge, or what actually happens in practice, the lowering of negative charge. During the interval of the discharge, the effective electric charge dipole moment formed by the stored charge and its image below the conducting surface of the earth increases at the rate at which charge is deposited by the current. At the surface of the earth at distances large compared to the the length of the effective electric dipole the direction of the electric field is downward, i.e., negative. It is the projection of this effective dipole field onto the normal mode electric fields that determines the relative excitation efficiency of each of the normal modes. We note that the overall magnitude of the electric field is linearly related to the duration of the driving current, and thus to the total charge moment of the event.

In the case of lightning excitation, the characteristic discharge time $T$ is on the order of $500 \mu \mathrm{s}$. For the Schumann resonances the lowest frequency is at roughly $7-8 \mathrm{~Hz}$ corresponding to $\omega_{n} \sim 50$ and $\gamma_{n} \sim 5$. The quantity $\omega_{n} T<<1$ may therefore be ignored in expression (25). The transient term $e^{-t / T}$ in this case decays over a period of roughly $500 \mu \mathrm{s}$, a much smaller time interval than can be resolved with the ELF monitoring systems that were deployed, and may be ignored. We may therefore write for a vertical lightning discharge with a few hundred microseconds duration

$$
E(\theta, t) \approx \frac{-I_{o} d s T}{4 \pi a^{2} h \epsilon_{o}} \sum_{n=0}^{\infty}(2 n+1) e^{-\gamma_{n} t} \cos \left(\omega_{n} t\right) P_{n}(\cos \theta)
$$

At the opposite limit of characteristic discharge times greater than the normal mode periods of the cavity, i.e., $\omega_{n} T>1$, and again ignoring the transient 
response, the expression for the electric field becomes

$$
E(\theta, t) \approx \frac{-I_{o} d s T}{4 \pi a^{2} h \epsilon_{o}} \sum_{n=0}^{\infty}(2 n+1) \frac{e^{-\gamma_{n} t}}{\omega_{n} T} \sin \left(\omega_{n} t\right) P_{n}(\cos \theta) .
$$

The inphase and quadrature terms are equal when $\omega_{n} T=1$.

Comparing expressions (26) and (27), we see that a current with a finite duration greater than the wave period will excite the cavity less efficiently per unit charge moment than an impulsive current of the same initial amplitude. This behavior may be traced to the fact that in an extended discharge the excitation occurs continuously throughout the life of the current. Thus, waves excited during the latter portions of the discharge may be out of phase with those excited during earlier portions of the discharge, and the longer the discharge the more phase cancellation will occur. Indeed, for long enough excitation times the excitation strength becomes independent of the length of the discharge and only a function of current moment.

The relevance of this analysis comparing the effects of short and long duration sources lies on the fact that, in addition to being weak and unobservable at distances beyond roughly $5-10 \mathrm{~km}$, the electric fields generated by the underground tests at the Nevada Test Site were observed to persist for characteristic periods of several hundred ms. These relatively long periods are comparable to or greater than the wave period of the lowest Schumann mode. Thus, the current moment $I_{o} d s$ equivalent to that computed from the observed maximum rate of change in the vertical electric field near the test would excite the cavity less than an impulsive lightning source of the same magnitude.

\section{Background Noise: Application to Lightning}

We may estimate roughly the expected magnitudes of the various terms in the above expressions. Let us take as an example an average lightning stroke of initial magnitude $20,000 \mathrm{~A}$, length $1 \mathrm{~km}$, and duration $T=500 \mu \mathrm{s}$. Inserting values for the radius of the earth and the free space permittivity, and assuming that the height of the ionosphere at ELF is $50 \mathrm{~km}$, expression (26) becomes

$$
E(\theta, t) \approx-4.4 \times 10^{-5} \sum_{n=0}^{\infty}(2 n+1) e^{-\gamma_{n} t} \cos \left(\omega_{n} t\right) P_{n}(\cos \theta) .
$$


If we assume that a good approximation for the average value of $P_{n}$ is its $r m s$ value over the interval $[0, \pi]$, the average value of $\cos \left(\omega_{n}\right)$ is 0.5 , the average value of the exponential damping factor over a 1 -second interval is $1 / \gamma_{n}$, and that the bandpass of the measurement instrumentation encompasses the lowest five resonances, then the average signal level of our example lightning stroke over a $1 \mathrm{~s}$ interval is roughly $20 \mu \mathrm{V} / \mathrm{m}$. If we now assume an average global lightning discharge rate of $100 / \mathrm{s}$ and that the excited modes from each of the individual strokes add incoherently, then the average amplitude of the background electric field is approximately $200 \mu \mathrm{V} / \mathrm{m}$. The corresponding average magnetic field amplitude may be approximated by $B=E / v_{p h}$, where $v_{p h} \sim 0.7 c$ is the phase speed of the waves in the earth-ionosphere cavity. This corresponds to an average background magnetic field of approximately $1 \mathrm{pT}$.

The average electric and magnetic field backgrounds derived using the above approximations are in reasonable agreement with what is observed experimentally. There is a strong diurnal modulation of the electric and magnetic backgrounds by a factor of 2-3, with maximum amplitudes occurring near 2100 UT (1300 PST). Thus, the electric field backgrounds were typically $300-500 \mu \mathrm{V} / \mathrm{m}$ during the time periods when measurements were performed at the Nevada Test Site.

\section{Application to Fields Generated by an Underground Test}

We now estimate the equivalent vertical current dipole moment generated by the underground test. The maximum distance at which the fields, either electric or magnetic, were observed was approximately $5-10 \mathrm{~km}$, at which point they fell to the background level. The background levels of the Schumann resonance electric field during a typical test were roughly $500 \mu \mathrm{V} / \mathrm{m}$. If we take the characteristic time scale for the change of the observed electric field to be $250 \mathrm{~ms}$, as observed at close range, the time rate of change at $5 \mathrm{~km}$ corresponding to a signal at the background level is $2 \times 10^{-3} \mathrm{~V} / \mathrm{m}-\mathrm{s}$. Assuming the measured near field was of electrostatic origin and possesses an inverse-cube fall off with distance, this is equivalent to a time rate of change in the source charge dipole moment of $5 \times 10^{8}$ C $-\mathrm{m} / \mathrm{s}$ at a range of $5 \mathrm{~km}$, which is a current dipole moment of $5 \times 10^{8} \mathrm{~A}-\mathrm{m}$. By way of comparison a typical 20,000 A lightning discharge of length $1 \mathrm{~km}$ will have a current moment of $2 \times 10^{8} \mathrm{~A}-\mathrm{m}$. Thus, the observed electric fields are consistent with having effective current moments comparable to those of typical single lightning strokes. 
However, the characteristic scale time of $250 \mathrm{~ms}$ for the equivalent current moment is larger than that of lightning by a factor of roughly 500 , so the spectral energy of the source is concentrated at much lower frequencies than for lightning. In this case the quantity $\omega_{n} T$ in the denominator of expression (27) has a value of roughly 5 for the lowest mode, leading to an estimated vertical field amplitude of roughly $4 \mu \mathrm{V} / \mathrm{m}$ and a corresponding magnetic field of $20 \mathrm{fT}$ at distant locations. When these values are compared to the measured background fields of $500 \mu \mathrm{V} / \mathrm{m}$ and $1 \mathrm{pT}$, respectively, it is therefore not surprising that no signals were observed from the remote ELF measurement sites that were deployed at Livermore, Table Mountain and Alaska during several of the tests.

We conclude that the absence of detectable ELF electromagnetic signals at locations distant from the underground nuclear tests is consistent with theoretical estimates of expected signal strengths based on measurements of the near field excitation source characteristics. In view of the fact that several of the tests monitored in this program may have been near the upper limit of 150 kilotons permitted by the test ban treaty, the absence of readily detectable ELF signals at large distances makes the use of these techniques questionable for long range verification monitoring. 


\section{Bibliography}

Bliokh, P.V., A.P. Nikolaenko, and Yu. F. Filippov, Schumann Resonances in the Earth-Ionosphere Cavity, Inst. of Elec. Eng. Waves Series, Peter Peregrinus, London, 1980.

Galejs, J., Terrestrial Propagation of Long Electromagnetic Waves, Pergamon, New York, 1972.

Polk, C., "Schumann Resonances," in CRC Handbook of Atmospherics, Vol. 1, CRC Press, Boca Raton, 1982.

Wait, J.R., Electromagnetic Waves in Stratified Media, Macmillan, New York, 1962. 